\title{
LAS PROPIEDADES DE TIPO CONVERGENCIA EN ESPACIOS DE FUNCIONES
}

\author{
tesis que presenta \\ José Alfonso Ramírez de Arellano Novoa \\ para obtener el grado académico de \\ Maestro en Ciencias (Matemáticas) \\ dirigida por \\ el Dr. Vladimir Tkachuk
}


LAS PROPIEDADES DE TIPO CONVERGENCIA EN ESPACIOS DE FUNCIONES

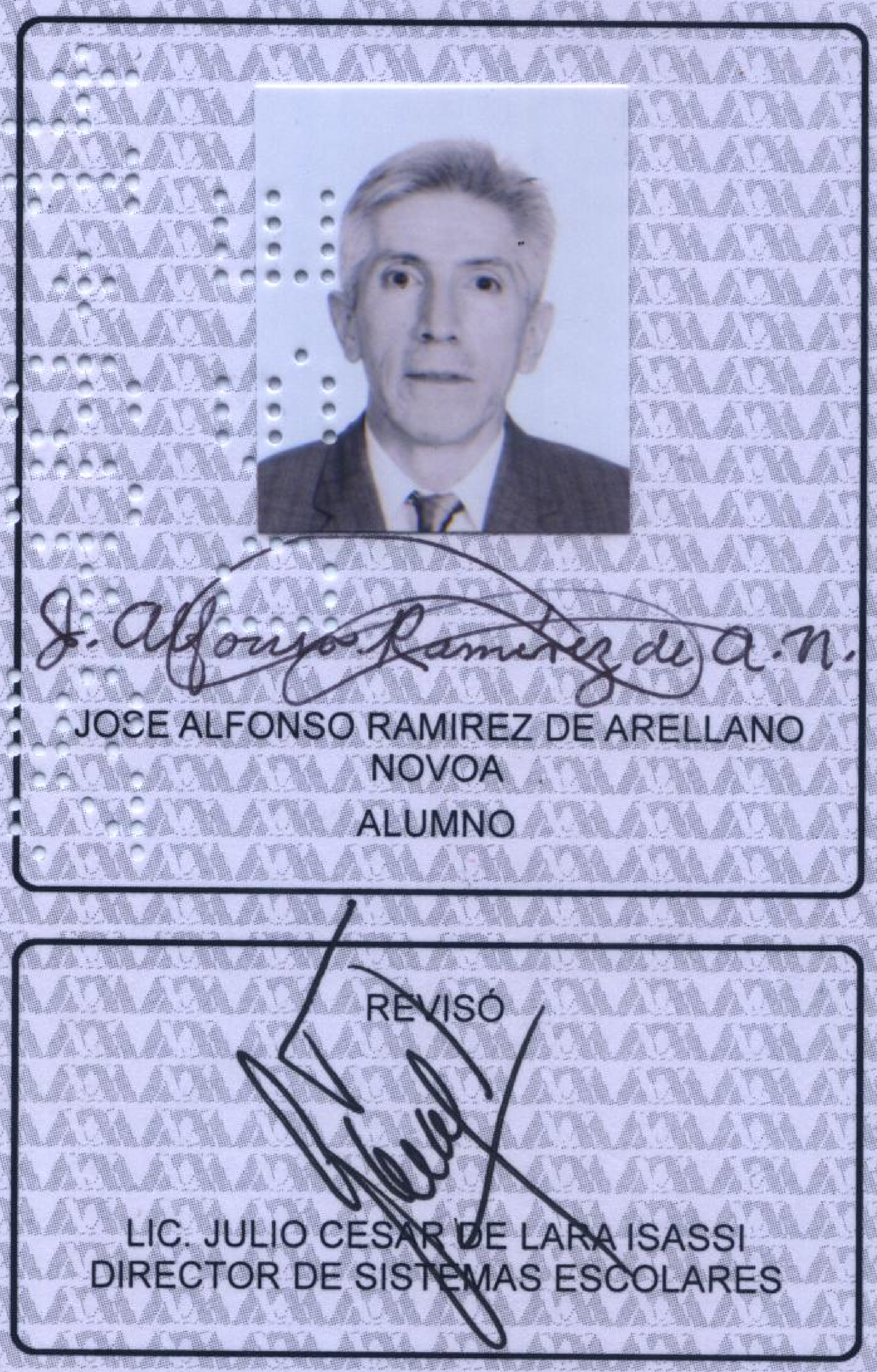

Bajo la Presidencia del primero y con carácter de Secretario el último, se reunieron para proceder al Examen de Grado cuya denominación aparece al margen, para la obtención del grado de:

MAESTRO EN CIENCIAS (MATEMÁTICAS)

DE: JOSE ALFONSO RAMIREZ DE ARELLANO NOVOA

y de acuerdo con el artículo 78 fracción III del Reglamento de Estudios Superiores de la Universidad Autónoma Metropolitana, los miembros del jurado resolvieron:

\section{aprobar}

Acto continuo, el presidente del jurado comunicó al interesado el resultado de la evaluación $y$, en caso aprobatorio, le fue tomada la protesta.
DIRECTOR DE LA DIVISIÓN DE CBI

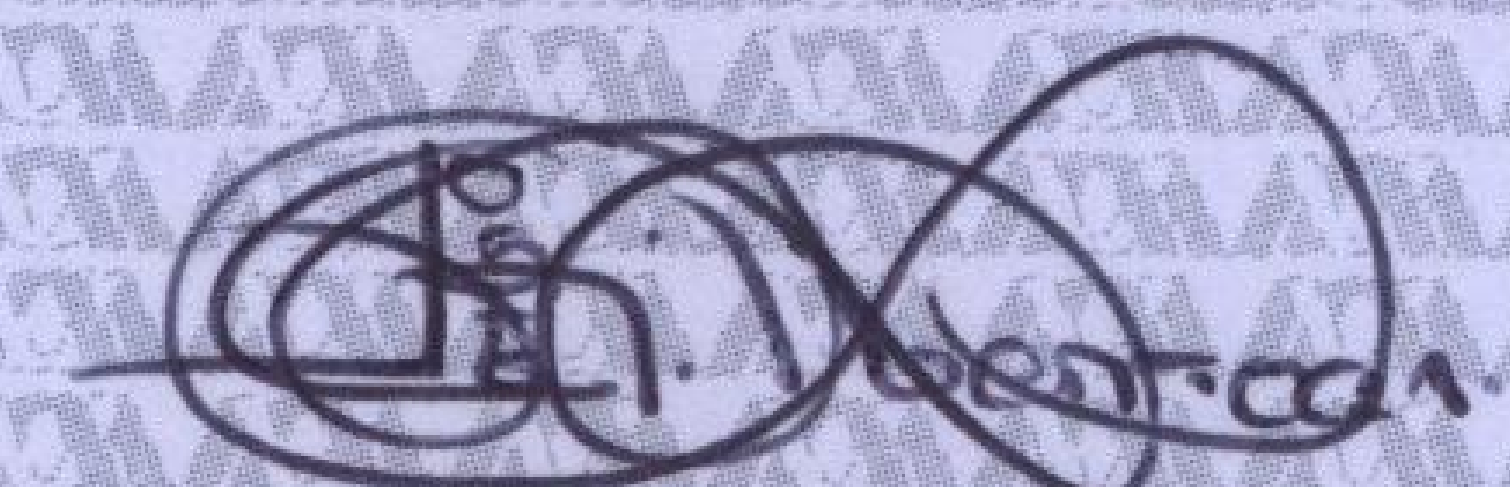

DR. JOSE GILBERTO CORDOBA HERRERA

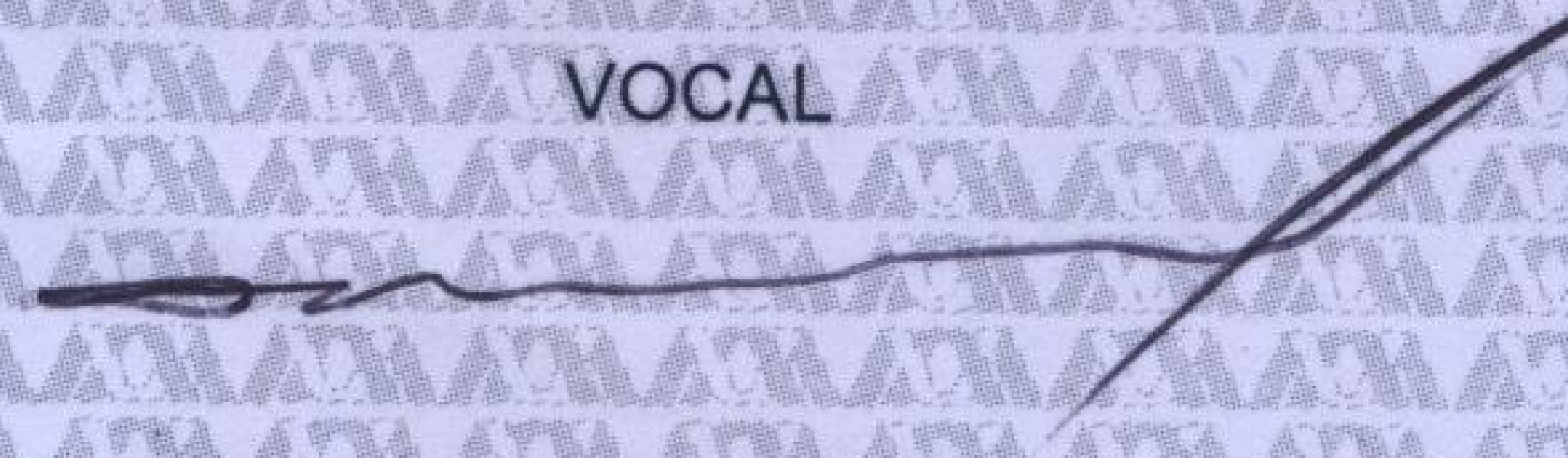

DR. OLEG OKUNEV GENNADIEVICH

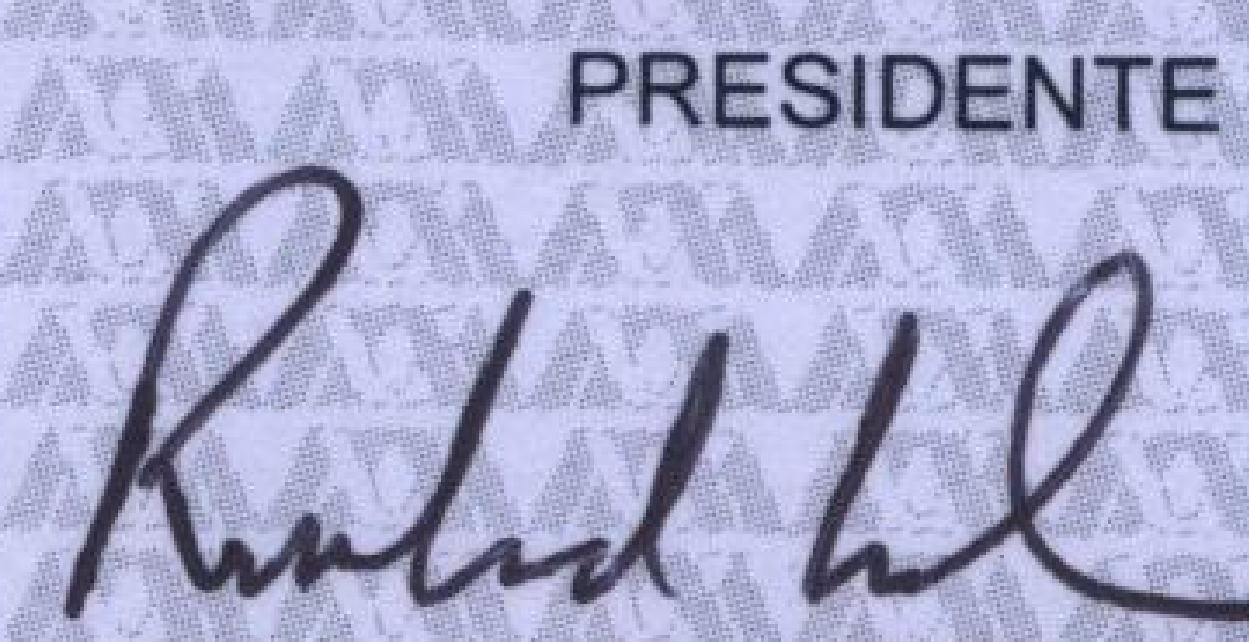

DR. RICHARD GORDONWILSON ROBERTS

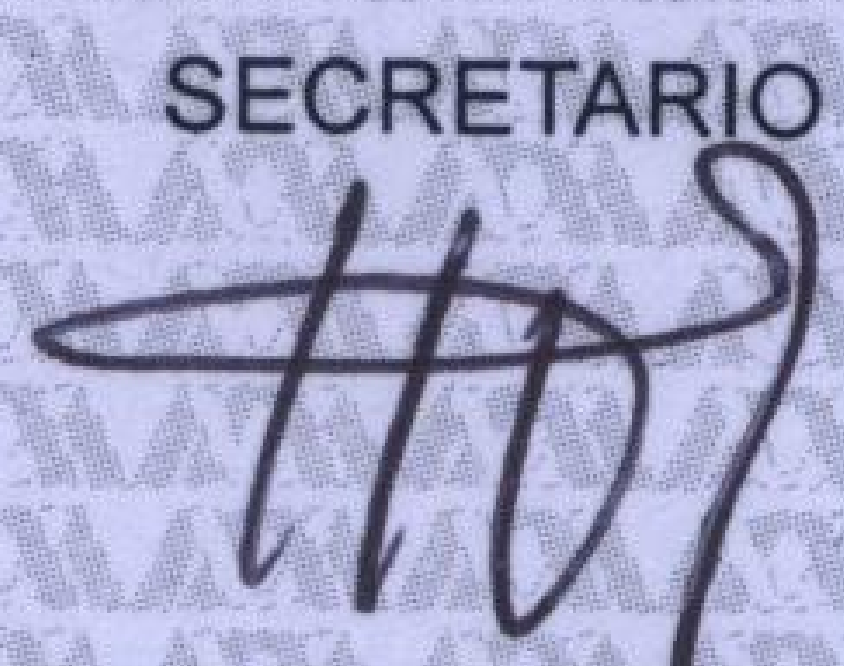

DR. VLADIMIR TKATCHOUK VLADIMIROVICH 


\section{Contenido}

Resumen . . . . . . . . . . . . . . . . . . . . . . . . . . . . iii

Introducción . . . . . . . . . . . . . . . . . . . . . . . . . . . iv

Capítulo 1. Propiedades de convergencia en espacios topológicos generales . . . . . . 1

1.1. Clasificación de espacios en términos de carácter y pseudocarácter . . . . . . 1

1.2. Sucesiones convergentes y sus generalizaciones . . . . . . . . . . . . . 5

1.3. Propiedades categóricas: teoremas y ejemplos . . . . . . . . . . . . . . . 13

Capítulo 2. Propiedades de convergencia en espacios de funciones . . . . . . . . . 21

2.1. Restricciones que implica la estructura algebraica de $C_{p}(X)$. . . . . . . . . 21

2.2. Caracterizaciones de propiedades de convergencia en $C_{p}(X)$. . . . . . . . . . $\quad 33$

2.3. El teorema de Gerlits-Pytkeev . . . . . . . . . . . . . . . . . . 41

Conclusiones y perspectivas

Bibliografía . . . . . . . . . . . . . . . . . . . . . . . . . . . . . . . . . . 49

Índice . . . . . . . . . . . . . . . . . . . . . . . . . . . . . . 52 


\section{Resumen}

En este trabajo se consideran las siguientes propiedades de convergencia: carácter, pseudocarácter, Fréchet-Urysohn, secuencialidad y estrechez. Primero en el contexto de los espacios topológicos generales, se analizan sus relaciones y las que tienen con las operaciones en espacios topológicos: toma de subespacios, producto y paso a espacios cocientes; también se caracterizan carácter numerable, Fréchet-Urysohn y secuencialidad en términos de espacios métricos. El material presentado es estándar. Después se estudian las mismas propiedades en el contexto de los espacios de funciones $C_{p}(X)$ los cuales están dotados con la topología de convergencia puntual y con cierta estructura algebraica; asimismo, se analizan sus relaciones con algunas operaciones en espacios topológicos. Por otro lado, se establecen los principales teoremas de dualidad entre un espacio $X$ y su correspondiente espacio $C_{p}(X)$, que involucran las propiedades de convergencia; además, se presenta el teorema más importante sobre propiedades de convergencia en espacios $C_{p}(X)$. Parte del material presentado aquí es estándar y parte constituye una porción de la frontera de lo que se

conoce en esta área. 


\section{Introducción}

Es un teorema clásico del Análisis que la cerradura de cualquier subconjunto de $\mathbb{R}$ se determina por medio de sucesiones convergentes, es decir, para todo conjunto $A \subset \mathbb{R}$ un punto $x$ pertenece a la cerradura de $A$ si y solo si existe una sucesión $\left\{a_{n}\right\} \subset A$ que converge a $x$. En los cursos avanzados de Análisis se prueba que lo mismo es cierto para cualquier espacio métrico.

Pero los objetos de estudio del Análisis no necesariamente son espacios métricos. En el Análisis Funcional se estudian espacios topológicos lineales, mismos que no siempre tienen la topología determinada por sucesiones convergentes; para verlo, basta considerar el espacio lineal topológico $\mathbb{R}^{\omega_{1}}$ y su subespacio $\Sigma$ de los puntos que tienen a lo más una cantidad numerable de coordenadas distintas de cero. El conjunto $\Sigma$ es denso en $\mathbb{R}^{\omega_{1}}$, pero si $u \in \mathbb{R}^{\omega_{1}}$ es el punto con todas sus coordenadas iguales a uno, entonces ninguna sucesión contenida en $\Sigma$ puede converger a $u$.

Este ejemplo ilustra varias cosas importantes; en primer lugar, vemos que fuera de los espacios métricos (y hasta en los espacios lineales topológicos) no podemos esperar que la cerradura de cada conjunto se determine por sucesiones convergentes. Los espacios respectivos se llaman FréchetUrysohn, así que vemos que no todos los espacios lineales topológicos son Fréchet-Urysohn. En el contexto de Topología General, el espacio $\{0,1\}^{\omega_{1}}$ muestra que la compacidad de un espacio topológico no necesariamente implica la propiedad de Fréchet-Urysohn.

Dicha situación conlleva dos maneras de desarrollar la investigación de las propiedades de convergencia. Se llevó a cabo un estudio detallado de la clase de los espacios Fréchet-Urysohn en diferentes contextos para delinear la frontera de esta clase y descubrir sus propiedades categóricas; se demostró entre otras cosas que cualquier compacto de Corson es Fréchet-Urysohn, que la clase de los espacios Fréchet-Urysohn es invariante para subespacios y bajo mapeos pseudoabiertos. Además, se logró dar una caracterización completa de los espacios Fréchet-Urysohn por medio de espacios métricos; resulta que un espacio $X$ es Fréchet-Urysohn si y solo si $X$ es una imagen pseudoabierta de un espacio métrico.

Otra manera de estudiar las propiedades de convergencia es considerar una propiedad más débil que la de Fréchet-Urysohn y delinear los límites de la clase correspondiente; así es como se descubrieron las clases de espacios secuenciales y los espacios de estrechez numerable. Un espacio $X$ es secuencial si para todo conjunto no cerrado $A \subset X$ se puede encontrar una sucesión $\left\{a_{n}\right\} \subset A$ que converge a un punto fuera de $A$. El espacio $X$ tiene estrechez numerable si para todo $A \subset X$ y todo $x \in \bar{A}$ existe un conjunto numerable $B \subset A$ tal que $x \in \bar{B}$.

Es fácil verificar que cada espacio Fréchet-Urysohn es secuencial, pero no es inmediato demostrar que todo espacio secuencial tiene estrechez numerable. Tampoco son triviales los ejemplos 
de espacios no secuenciales de estrechez numerable y de los espacios secuenciales que no son FréchetUrysohn.

Tanto la estrechez numerable como la secuencialidad se conservan bajo mapeos cocientes; cualquier subespacio de un espacio de estrechez numerable también tiene estrechez numerable. La afirmación análoga no es cierta para los espacios secuenciales; de hecho, un espacio hereditariamente secuencial tiene que ser Fréchet-Urysohn. La teoría que trata sobre los espacios de Fréchet-Urysohn y su relación con los espacios secuenciales y de estrechez numerable es muy rica; para convencernos de ello basta mencionar los siguientes hechos:

- Existen modelos de la teoría usual de conjuntos (ZFC) en los cuales cada espacio compacto de estrechez numerable es secuencial.

- Existen modelos de ZFC en los cuales hay espacios compactos de estrechez numerable que no son secuenciales.

- En los espacios $C_{p}(X)$ la propiedad de Fréchet-Urysohn y la secuencialidad coinciden.

- Existen grupos topológicos numerables secuenciales no metrizables.

- Es un viejo problema abierto determinar si cada grupo topológico numerable de FréchetUrysohn es metrizable.

También existe una caracterización de espacios secuenciales por medio de espacios métricos, misma que dice que un espacio $X$ es secuencial si y solo si $X$ es una imagen cociente de un espacio métrico.

Tradicionalmente, la propiedad de tener una base local numerable en cada punto también se considera propiedad de convergencia; los espacios respectivos se llaman primero numerables. El concepto de un espacio primero numerable surgió de la idea de generalizar las propiedades de la familia de las bolas de radios racionales alrededor de un punto en un espacio métrico; los espacios primero numerables no necesitan ser métricos pero son Fréchet-Urysohn. La propiedad de ser primero numerable es hereditaria y se conserva bajo mapeos abiertos; además, un grupo topológico primero numerable tiene que ser metrizable de modo que lo mismo se cumple para los espacios $C_{p}(X)$ o más generalmente para los espacios lineales topológicos. Igual que en el caso de espacios secuenciales y de Fréchet-Urysohn, se puede caracterizar a los espacios primero numerables por medio de espacios métricos; resulta que un espacio es primero numerable si y solo si es una imagen abierta de un espacio métrico.

De acuerdo con lo anterior se observa que en los espacios $C_{p}(X)$ las propiedades de tipo convergencia mejoran notablemente; asimismo, ciertas propiedades de convergencia se pueden caracterizar en términos de otras que no lo son a diferencia de lo que sucede en espacios topológicos generales $X$; así, para todo $X$, tenemos las desigualdades $\chi(X) \leq w(X)$ y $\psi(X) \leq i w(X)$ (ver definición 2.2.4), pudiéndose hallar ejemplos en los que las desigualdades son estrictas, en tanto que $\chi\left(C_{p}(X)\right)=w\left(C_{p}(X)\right)$ y $\psi\left(C_{p}(X)\right)=i w\left(C_{p}(X)\right)$. Por otro lado, un espacio $C_{p}(X)$ es Fréchet- 


\section{vi Introducción}

Urysohn si y solo si es un espacio $k$, lo que involucra una propiedad que no es de tipo convergencia.

El principal propósito de la tesis es el estudio de las propiedades de tipo convergencia en los espacios $C_{p}(X)$ y la comparación del comportamiento de dichas propiedades en espacios topológicos generales; como consecuencia de lo anterior se llegará a la frontera del conocimiento.

En el primer capítulo se estudian las propiedades de convergencia en el contexto general. En la primera sección se consideran las propiedades más elementales, el carácter y el pseudocarácter; se analiza la relación que tienen entre sí y con ciertas operaciones en espacios topológicos, como son la toma de subespacios, el producto y el paso a espacios cocientes. Además, se distinguen algunas clases de espacios por la relación especial que tienen las propiedades en ellos. Los enunciados más importantes son los teoremas 1.1.10, 1.1.11 y 1.1.13.

En la segunda sección se consideran las propiedades de convergencia como Fréchet-Urysohn, secuencialidad y estrechez numerable; también se describen algunas relaciones sencillas entre ellas. Los principales enunciados de esta sección son los teoremas 1.2.9, 1.2.13, 1.2.17 y 1.2.20.

En la tercera sección se analiza la relación entre las propiedades carácter numerable, FréchetUrysohn, secuencialidad y estrechez numerable con las operaciones topológicas antes mencionadas; asimismo, se proporcionan caracterizaciones de las tres primeras propiedades en términos de espacios métrizables y se presenta otra relación entre las propiedades Fréchet-Urysohn y secuencialidad. Los principales enunciados de la sección son los teoremas 1.3.13, 1.3.15, 1.3.17 y 1.3.22.

En el segundo capítulo se estudian las propiedades de convergencia en espacios de funciones dotados con la topología de convergencia puntual y con alguna estructura algebraica. En la primera sección se definen las estructuras algebraicas y se analiza su relación con la topología, para obtener a partir de un espacio $X$ (o $(X, Y)$ ), el espacio de funciones $C_{p}(X)$ (o $C_{p}(X, Y)$ ) objeto de estudio; se considera al paso de $X$ a $C_{p}(X)$, o de $(X, Y)$ a $C_{p}(X, Y)$ como una operación topológica $\mathcal{C}$, se estudia su relación con otras operaciones, y de hecho se observa que tras aplicar la operación $\mathcal{C}$, algunas propiedades topológicas varían notablemente y las relaciones entre otras se enriquecen. Los principales enunciados son los teoremas 2.1.24 y 2.1.30.

En la segunda sección se examina con detalle el problema de determinar propiedades de convergencia de $C_{p}(X)$ a partir de propiedades de $X$ y viceversa; también se consideran algunas consecuencias de los teoremas presentados, en donde de nuevo se observan grandes diferencias entre un espacio $X$ y el correspondiente $C_{p}(X)$. Los principales enunciados son los teoremas 2.2.12 y 2.2.18.

El enunciado principal de la sección 2.3 es el teorema 2.3.10 que establece que en los espacios $C_{p}(X)$ la propiedad $k$ coincide con la secuencialidad y la propiedad de Fréchet-Urysohn.

Quisiera agradecer a los sinodales: el Dr. Oleg Okunev, el Dr. Vladimir Tkachuk y el Dr. Richard Wilson, por su cuidadosa revisión de la tesis e indicación de algunas mejoras, en especial al asesor el Dr. Tkachuk por su minuciosa supervisión y enorme paciencia durante todo el proceso de elaboración de la tesis; asimismo, por haberme mostrado una manera muy adecuada de redactar un texto matemático. También quisiera agradecer a la actual Coordinadora de Posgrado en Matemáticas de la UAMI, la Dra. Patricia Saavedra y al anterior Coordinador, el Dr. Raúl Montes de Oca por su apoyo y comprensión, y a la Asistente de Posgrado, la M. en C. Ma. Iseo González por sus múltiples consejos durante la Maestría. 


\section{CAPÍTULO 1}

\section{Propiedades de convergencia en espacios topológicos generales}

En este capítulo se introducen las propiedades de tipo convergencia tales como carácter, pseudocarácter, Fréchet-Urysohn, secuencialidad y estrechez. Se presentan algunos enunciados y ejemplos que muestran importantes facetas de las propiedades de convergencia, así como relaciones entre ellas. También se estudia el comportamiento de éstas al aplicar las operaciones producto, toma de subespacios y paso a espacios cocientes a los espacios topológicos que las poseen.

\subsection{Clasificación de espacios en términos de carácter y pseudocarácter}

A lo largo de toda la tesis se trabajará sólo con espacios de Tychonoff; las letras $X, Y, Z$ con o sin subíndices, representarán espacios topológicos, $\lambda$ y $\kappa$ representarán números cardinales (y sus correspondientes números ordinales), $\omega$ el menor cardinal infinito, $\omega_{1}$ el primer cardinal no numerable, $\kappa^{+}$el primer cardinal mayor que $\kappa$ y las letras $\mathbb{R}, \mathbb{Q}, \mathbb{N}$ los conjuntos de los números reales, racionales y naturales positivos respectivamente, con su topología usual a menos que se indique lo contrario. Para cualquier espacio $X$, su topología será $\tau(X)$; si además $A \subset X$, se denotará por $\tau(A, X)$ a la familia $\{U \in \tau(X): A \subset U\}$, aunque en vez de $\tau(\{x\}, X)$ se escribirá $\tau(x, X)$. Cada vez que $X$ sea un producto cartesiano se supondrá que $\tau(X)$ es la topología producto. Una sucesión ordinaria con términos $x_{n}$ se denotará por $\left\{x_{n}: n \in \mathbb{N}\right\},\left\{x_{n}: n \in \omega\right\}$ o por $\left\{x_{n}\right\}$ si está claro cual es el conjunto de índices; una sucesión transfinita con términos $x_{\alpha}$ se escribirá como $\left\{x_{\alpha}: \alpha<\kappa\right\}$ para algún $\kappa>\omega$.

En esta sección se analizan el carácter y pseudocarácter de un espacio, sus propiedades y relaciones; también se caracterizan algunas clases de espacios por las relaciones especiales que estas propiedades presentan en ellos.

1.1.1. Definición. Sea $A \subset X$; una familia $\mathcal{B} \subset \tau(A, X)$ es una base externa de $A$ en $X$ si para cada $U \in \tau(A, X)$ existe $V \in \mathcal{B}$ tal que $V \subset U$. El carácter de $A$ en $X$ es el cardinal $\chi(A, X)=\min \{|\mathcal{B}|: \mathcal{B}$ es una base externa de $A$ en $X\}$; en el caso particular $A=\{x\}$, se escribe $\chi(x, X)$. El pseudocarácter de $A$ en $X$ es el cardinal $\psi(A, X)=\min \{|\mathcal{U}|: \mathcal{U} \subset \tau(X)$ y $\bigcap \mathcal{U}=A\}$; aunque se escribe $\psi(x, X)$ cuando $A=\{x\}$. El carácter de $X$ es el cardinal $\chi(X)=$ 
$\sup \{\chi(x, X): x \in X\}$; el pseudocarácter de $X$ es el cardinal $\psi(X)=\sup \{\psi(x, X): x \in X\}$. Una condensación es un mapeo biyectivo continuo.

1.1.2. Proposición. (1) Para todo $X$, si $Y \subset X$, entonces $\chi(Y) \leq \chi(X)$; si además, $\bar{Y}=X$, tenemos la igualdad $\chi(y, Y)=\chi(y, X)$ para todo $y \in Y$.

(2) Si $|S| \leq \lambda \geq \omega y \chi\left(X_{s}\right) \leq \lambda$ para todo $s \in S$, entonces $\chi\left(\prod_{s \in S} X_{s}\right) \leq \lambda$.

(3) Si $f: X \rightarrow Y$ es un mapeo abierto sobreyectivo, se tiene que $\chi(Y) \leq \chi(X)$.

Demostración. (1) Sean $y \in Y$ y $\mathcal{B}_{y}$ una base local de $X$ en $y$ tal que $\left|\mathcal{B}_{y}\right|=\chi(y, X)$; entonces $\mathcal{A}=\left\{U \cap Y: U \in \mathcal{B}_{y}\right\}$ es una base local de $Y$ en $y$ para la cual $|\mathcal{A}| \leq \chi(y, X)$. Por tanto, $\chi(y, Y) \leq \chi(y, X)$, de donde $\chi(Y) \leq \chi(X)$. Si, además $\bar{Y}=X$, sea $\mathcal{A}_{y}$ una base local de $Y$ en $y$ que verifica $\left|\mathcal{A}_{y}\right|=\chi(y, Y)$; para cada $U \in \mathcal{A}_{y}$ elíjase $V_{U} \in \tau(X)$ tal que $U=V_{U} \cap Y$, y defínase $\mathcal{B}=\left\{V_{U}: U \in \mathcal{A}_{y}\right\}$. Tómese $O \in \tau(y, X)$; por ser $X$ regular, existe $W \in \tau(y, X)$ tal que $\bar{W} \subset O$. Luego existe $U \in \mathcal{A}_{y}$ que cumple $U \subset W \cap Y$; como $\bar{Y}=X$, se tiene que $U=V_{U} \cap Y$ satisface la condición $\bar{V}_{U}=\bar{U} \subset \bar{W} \subset O$. De modo que $y \in V_{U} \subset O$; de aquí $\mathcal{B}$ es una base local de $X$ en $y$. Por eso, $\chi(y, X) \leq|\mathcal{B}| \leq\left|\mathcal{A}_{y}\right|=\chi(y, Y)$, de donde $\chi(y, Y)=\chi(y, X)$.

(2) Sean $X=\prod_{s \in S} X_{s}, x=\left(x_{s}\right)_{s \in S} \in X$ y $\mathcal{B}_{s}$ una base local de $X_{s}$ en $x_{s}$ para la cual $\left|\mathcal{B}_{s}\right| \leq \lambda$ para todo $s$; entonces la familia $\mathcal{B}=\left\{\prod_{s \in S} U_{s}\right.$ : existe $F \subset S$ finito tal que $U_{s}=X_{s}$ para $s \in S \backslash F$ y $U_{s} \in \mathcal{B}_{s}$ para $\left.s \in F\right\}$ satisface las condiciones $\mathcal{B} \subset \tau(X)$ y $|\mathcal{B}| \leq \lambda$. Dado $U \in \tau(x, X)$ existe un conjunto $V=\prod_{s \in S} V_{s}$ tal que $x \in V \subset U$ y $V_{s}=X_{s}$ para todo $s$ excepto para $s$ en cierto conjunto finito $F$; puesto que $\mathcal{B}_{s}$ es una base local de $X_{s}$ en $x_{s}$ para cualquier $s$, existe $B_{s} \in \mathcal{B}_{s}$ que cumple $x_{s} \in B_{s} \subset V_{s}$ para cada $s \in F$. Por eso, si $B_{s}=X_{s}$ para todo $s \in S \backslash F$ se obtiene $B=\prod_{s \in S} B_{s} \in \mathcal{B}$ y $x \in B \subset V$. Por tanto, $\mathcal{B}$ es una base local de $X$ en $x$, de donde $\chi(x, X) \leq \lambda$, así que $\chi(X) \leq \lambda$.

(3) Sea $\mathcal{B}$ una base local de $X$ en $x$; entonces $f(\mathcal{B})=\{f(U): U \in \mathcal{B}\}$ es una base local de $Y$ en $f(x)$ y $|f(\mathcal{B})| \leq|\mathcal{B}|$. Por tanto, $\chi(Y) \leq \chi(X)$.

1.1.3. Corolario. Si $f: X \rightarrow Y$ es un mapeo abierto sobreyectivo $Y$ y $X$ es metrizable, entonces $\chi(Y) \leq \omega$.

Demostración. Es consecuencia de la proposición 1.1.2 y de que cada espacio metrizable $X$ cumple $\chi(X) \leq \omega$.

\subsubsection{Proposición. Para todo $X$, si $A \subset X$ entonces $|\bar{A}| \leq|A|^{\chi(X)}$.}

Demostración. Para cada conjunto $B$ sea $P_{\kappa}(B)=\{C \subset B:|C| \leq \kappa\}$. Hágase $\chi(X)=\kappa$ y fijemos una base local $\mathcal{B}_{x}$ tal que $\left|\mathcal{B}_{x}\right| \leq \kappa$ en cada punto $x \in X$. Si $\kappa<\omega$, por ser $X$ un espacio $T_{1}$, se deduce que $\bigcap \mathcal{B}_{x}=\{x\}$ para todo $x \in X$; luego $X$ es discreto, de donde $A=\bar{A}$ para todo $A \subset X$ y $|\bar{A}| \leq|\bar{A}|^{\kappa}=|A|^{\kappa}$. Si $\kappa \geq \omega$, tenemos que $\left|P_{\kappa}(A)\right| \leq|A|^{\kappa}$; de aquí $\left|P_{\kappa}\left(P_{\kappa}(A)\right)\right| \leq|A|^{\kappa \cdot \kappa}=|A|^{\kappa}$. Por eso, es suficiente construir una inyección $\phi: \bar{A} \rightarrow P_{\kappa}\left(P_{\kappa}(A)\right)$; dado $x \in \bar{A}$, elíjase $x_{U} \in U \cap A$ para cada $U \in \mathcal{B}_{x}$ y sea $C_{x}=\left\{x_{U}: U \in \mathcal{B}_{x}\right\}$. De esta manera, $C_{x} \subset A, x \in \bar{C}_{x}$ y $\left|C_{x}\right| \leq \kappa$; por tanto, $\mathcal{D}_{x}=\left\{C_{x} \cap U: U \in \mathcal{B}_{x}\right\} \in P_{\kappa}\left(P_{\kappa}(A)\right)$. Defínase $\phi(x)=\mathcal{D}_{x}$ para cada $x \in \bar{A}$; como $X$ es $T_{2}$ y $x \in \overline{C_{x} \cap U}$ para cada $U \in \mathcal{B}_{x}$, se tiene que $x \in \bigcap\left\{\bar{D}: D \in \mathcal{D}_{x}\right\} \subset \bigcap\left\{\bar{U}: U \in \mathcal{B}_{x}\right\}=\{x\}$. Por eso, $\{x\}=\bigcap\left\{\bar{D}: D \in \mathcal{D}_{x}\right\}$; de aquí se deduce que $\phi$ es inyectiva.

1.1.5. Proposición. (1) Para todo $X$, si $Y \subset X$, entonces $\psi(Y) \leq \psi(X)$. 
(2) Si $|S| \leq \lambda \geq \omega$ y $\psi\left(X_{s}\right) \leq \lambda$ para todo $s \in S$, entonces $\psi\left(\prod_{s \in S} X_{s}\right) \leq \lambda$.

Demostración. (1) Sean $x \in Y$ y $\mathcal{U} \subset \tau(X)$ tales que $\bigcap U=\{x\}$; entonces $\mathcal{U}^{\prime}=\{U \cap Y: U \in \mathcal{U}\} \subset$ $\tau(Y)$ y $\cap \mathcal{U}^{\prime}=\{x\}$, así que $\psi(x, Y) \leq\left|\mathcal{U}^{\prime}\right| \leq|\mathcal{U}|$. En particular, si elegimos $\mathcal{U}$ tal que $|\mathcal{U}|=\psi(x, X)$ se obtiene $\psi(Y) \leq \psi(X)$.

(2) Sean $X=\prod_{s \in S} X_{s}, x=\left(x_{s}\right)_{s \in S} \in X$ y $\mathcal{U}_{s} \subset \tau\left(X_{s}\right)$ tales que $\bigcap \mathcal{U}_{s}=\left\{x_{s}\right\}$ para todo s; entonces la familia $\mathcal{U}=\left\{\prod_{s \in S} U_{s}\right.$ : existe $F \subset S$ finito tal que $U_{s}=X_{s}$ para $s \in S \backslash F$ y $U_{s} \in \mathcal{U}_{s}$ para $s \in F\}$ satisface las condiciones $\mathcal{U} \subset \tau(X),|\mathcal{U}| \leq \lambda$ y $\cap \mathcal{U}=\{x\}$. De modo que $\psi(x, X) \leq \lambda$; por ello, $\psi(X) \leq \lambda$.

Al comparar las proposiciones 1.1.2 y 1.1.5, debe tenerse en cuenta que la analogía no es completa; en el capítulo 2, se dará un ejemplo de espacios $X, Y$ y un mapeo abierto sobreyectivo $f: X \rightarrow Y$ tales que $\psi(X)=\omega<\psi(Y)$ (ejemplo 2.2.7). No obstante se cumple el siguiente enunciado .

1.1.6. Proposición. Si $f: X \rightarrow Y$ es una condensación, entonces $\psi(X) \leq \psi(Y)$.

Demostración. Sean $x \in X, y=f(x)$ y $\mathcal{B}_{y} \subset \tau(Y)$ tal que $\{y\}=\bigcap \mathcal{B}_{y}$ y $\left|\mathcal{B}_{y}\right|=\psi(y, Y)$; entonces la familia $f^{-1}\left(\mathcal{B}_{y}\right)=\left\{f^{-1}(U): U \in \mathcal{B}_{y}\right\}$ satisface la condición $f^{-1}\left(\mathcal{B}_{y}\right) \subset \tau(x, X)$. Por ser $f$ uno a uno, $\bigcap f^{-1}\left(\mathcal{B}_{y}\right)=\{x\}$ y como $\left|f^{-1}\left(\mathcal{B}_{y}\right)\right|=\left|\mathcal{B}_{y}\right|$, se tiene que $\psi(x, X) \leq\left|f^{-1}\left(\mathcal{B}_{y}\right)\right| \leq \psi(y, Y)$; por lo tanto, $\psi(X) \leq \psi(Y)$.

1.1.7. Proposición. Para todo $x \in X$, se verifica que $\psi(x, X) \leq \chi(x, X)$.

Demostración. Sean $x \in X$ y $\mathcal{B}$ una base local de $X$ en $x$; entonces $\bigcap \mathcal{B}=\bigcap \tau(x, X)$ y puesto que $\bigcap \tau(x, X)=\{x\}$ se obtiene $\psi(x, X) \leq \chi(x, X)$.

Puede suceder que para un espacio $X$, sea $\psi(x, X)<\chi(x, X)$ en algún punto $x$.

1.1.8. Ejemplo. Sea $X=\{p\} \cup \bigcup_{\alpha<\kappa} X_{\alpha}$, donde $p \notin X_{\alpha}=\left\{x_{i}^{\alpha}: i \in \mathbb{N}\right\}$ para todo $\alpha$ y $x_{i}^{\alpha} \neq x_{j}^{\beta}$ para $(i, \alpha) \neq(j, \beta)$; se definirá $\tau(X)$ mediante la familia $\mathcal{B}=\mathcal{B}_{p} \cup\left\{\left\{x_{i}^{\alpha}\right\}: i \in \mathbb{N}\right.$ y $\left.\alpha<\kappa\right\}$, donde $\mathcal{B}_{p}$ consiste de todos los conjuntos obtenidos de $X$ al quitar un número finito de puntos $x_{i}^{\alpha}$ de cada uno de los conjuntos $X_{\alpha}$. Fácilmente se verifica que $\mathcal{B}$ es una base para una topología $T_{1}$ en $X$; además, es claro que cada $U \in \mathcal{B}$ es un conjunto cerrado. Por tanto, $X$ resulta ser un espacio de Tychonoff; el espacio $X$ se conoce como erizo de Urysohn con $\kappa$ espinas, siendo sus espinas los conjuntos $\{p\} \cup X_{\alpha}$ para todo $\alpha$. En particular, si $\kappa=\omega$ con facilidad se obtiene que $\psi(p, X)=\omega$; sin embargo, si $\mathcal{A}=\left\{V_{n}: n \in \mathbb{N}\right\} \subset \tau(p, X)$, puede elegirse $z_{n} \in V_{n} \cap X_{n}$, así que $U=X \backslash\left\{z_{n}: n \in \mathbb{N}\right\}$ satisface las condiciones $U \in \tau(p, X)$ y $V_{n} \not \subset U$ para todo $n$. Luego $\chi(p, X)>\omega$ lo cual muestra que la desigualdad en la proposición 1.1 .7 puede ser estricta.

Si $X$ es metrizable, es claro que $\chi(X) \leq \omega$; sin embargo, el recíproco no se cumple.

1.1.9. Ejemplo. Sea $\mathcal{B}=\{[x, x+\epsilon): x \in \mathbb{R}$ y $\epsilon>0\}$; se checa fácilmente que $\mathcal{B}$ es una base para una topología $T_{1}$ en $\mathbb{R}$ y que cada $U \in \mathcal{B}$ es un conjunto cerrado, por lo cual $\mathcal{B}$ determina un espacio de Tychonoff. El espacio $X$ resultante se llama recta de Sorgenfrey; como $\mathcal{B}$ es base de $X$, dado $x \in X$, se tiene por una parte que $\{[x, x+1 / n): n \in \mathbb{N}\}$ es una base local de $X$ en $x$. Por tanto, $\chi(x, X) \leq \omega$, así que $\chi(X) \leq \omega$. Por otra parte, si $\mathcal{A}$ es una base de $X$ y $x \in X$, debe existir $U \in \mathcal{A}$ cuyo elemento mínimo sea $x$; por tanto, $\mathcal{A}$ no es numerable lo que implica que $w(X)>\omega$. Es claro que el subespacio $\mathbb{Q}$ es denso en $X$, por lo cual $X$ es separable. Por lo tanto, $X$ no es metrizable. 
Una consecuencia de la proposición 1.1 .7 es que si $X$ es metrizable, para todo $x \in X$ se tiene que $\psi(x, X)=\chi(x, X)$. Puesto que $\chi(x, X) \leq \omega$, en caso de que tengamos $\psi(x, X)<\omega$, deberá ser $\psi(x, X)=1$. Luego $x$ es punto aislado en $X$; por tanto, $\chi(x, X)=1$. Existen otras clases de espacios $X$ para los que se cumple $\psi(x, X)=\chi(x, X)$.

1.1.10. Teorema. Para todo espacio compacto $X$, si $F \subset X$ es cerrado, tenemos la igualdad $\psi(F, X)=\chi(F, X)$.

Demostración. Si $X$ es infinito, como es regular y $F$ es cerrado, $\bigcap \tau(F, X)=F$; luego, si $\mathcal{B}$ es una base externa de $F$ en $X$, se cumple $\bigcap \mathcal{B}=F$ así que $\psi(F, X) \leq|\mathcal{B}|$ y por lo tanto, $\psi(F, X) \leq \chi(F, X)$. Ahora, si $\psi(F, X)=\kappa$, sea $F=\bigcap_{s \in S} V_{s}$, donde $V_{s} \in \tau(X)$ para todo $s \in S$ y $|S| \leq \kappa$; por [Engelking, teorema 3.1.9] se tiene que $X$ es normal, de modo que para todo $s \in S$ existe $U_{s} \in \tau(F, X)$ tal que $\bar{U}_{s} \subset V_{s}$. Por tanto, $F=\bigcap_{s \in S} \bar{U}_{s}$ y cada $\bar{U}_{s}$ es compacto; por [Engelking, corolario 3.1.5], para cada $U \in \tau(F, X)$ existe $\left\{s_{1}, \ldots, s_{k}\right\} \subset S$ para el cual $U_{s_{1}} \cap \ldots \cap U_{s_{k}} \subset$ $\bar{U}_{s_{1}} \cap \ldots \cap \bar{U}_{s_{k}} \subset U$; luego, la familia $\mathcal{U}$ de todas las intersecciones finitas de elementos de $\left\{U_{s}: s \in S\right\}$ forma una base externa de $F$ en $X$. Puesto que $|\mathcal{U}| \leq \kappa$, se obtiene $\chi(F, X) \leq \psi(F, X)$. Si $X$ es finito, es discreto y claramente se da la igualdad.

1.1.11. Teorema. Si $X$ es linealmente ordenado y $x \in X$, entonces $\psi(x, X)=\chi(x, X)$.

Demostración. Sea $\leq$ un orden lineal en $X$ que genera $\tau(X)$ y tomemos $x \in X$ tal que $\psi(x, X)=\kappa$. Sea $\kappa \geq \omega$; podemos suponer que $x$ no es el mayor ni el menor elemento de $X$, ya que en los casos pertinentes la demostración que sigue se simplifica. Así, existe $\mathcal{B}=\left\{\left(a_{s}, b_{s}\right): s \in S\right\}$ que verifica $\{x\}=\bigcap \mathcal{B}$ y $|S|=\kappa$, donde $\left(a_{s}, b_{s}\right)=\left\{y \in X: a_{s}<y<b_{s}\right\}$ para todo $s \in S$; dado $U \in \tau(x, X)$ existen $a, b \in X$ para los cuales $x \in(a, b) \subset U$. Como $a \notin \bigcap \mathcal{B}$, existe $t \in S$ tal que $a \notin\left(a_{t}, b_{t}\right)$, luego $a \leq a_{t}$; asimismo, $b \notin \bigcap \mathcal{B}$ implica que existe $u \in S$ para el que $b \notin\left(a_{u}, b_{u}\right)$ por lo cual $b \geq b_{u}$. Por tanto, $W=\left(a_{t}, b_{t}\right) \cap\left(a_{u}, b_{u}\right)$ cumple $x \in W \subset\left(a_{t}, b_{u}\right) \subset(a, b) \subset U$, así que la familia $\mathcal{E}$ de todas las intersecciones finitas de elementos de $\mathcal{B}$ forma una base local para $X$ en $x$; puesto que $|\mathcal{E}| \leq \kappa$, se obtiene $\chi(x, X) \leq \kappa$. Por la proposición 1.1.7, tenemos que $\psi(x, X)=\chi(x, X)$. Ahora si $\kappa<\omega$, entonces $x$ es un punto aislado y también se verifica $\psi(x, X)=\chi(x, X)$.

Con respecto al teorema anterior debe tenerse en cuenta que no es necesario suponer que $X$ sea además espacio de Tychonoff, pues puede probarse que todo espacio linealmente ordenado es $T_{1}$ y normal por colecciones (ver [Tkachuk (2010), problema 302]).

1.1.12. Corolario. Si $X$ es compacto o linealmente ordenado, entonces $\chi(X) \leq|X|$.

Demostración. Sea $x \in X$; como $\{x\}=\bigcap\{X \backslash\{y\}: x \neq y \in X\}$, se deduce que $\psi(x, X) \leq|X|$. Por los teoremas 1.1.10 y 1.1.11, se cumple $\chi(X) \leq|X|$.

Considerando que los principales objetos de nuestro estudio son casos particulares de grupos topológicos, es bueno tener en cuenta el siguiente enunciado, el cual, no se probará (para una demostración, véase [Arhangel'skii y Tkachenko, teorema 3.3.12]); pues para el caso de los espacios $C_{p}(X)$ se demostrará mediante una herramienta de la teoría $C_{p}$ (corolario 2.2.3).

1.1.13. Teorema (Birkhoff-Kakutani). Si $G$ es un grupo topológico tal que $\chi(G) \leq \omega$, entonces $G$ es metrizable. 


\subsection{Sucesiones convergentes y sus generalizaciones}

En esta sección se consideran las propiedades Fréchet-Urysohn, secuencialidad y estrechez numerable; además se describen algunas relaciones sencillas entre ellas. También se presentan dos teoremas más profundos sobre espacios compactos en los que las propiedades de convergencia se relacionan con otras propiedades de manera especial; para el primero, que trata sobre espacios compactos diádicos, se utilizarán tres proposiciones y un importante teorema de descomposición de funciones continuas de A.V. Arhangel'skii. Para el segundo, que también es un importante teorema de A.V. Arhangel'skii, se empleará la herramienta ya presentada.

En cualquier espacio métrico $X$, si $x \in X, A \subset X$ y $x \in \bar{A}$, se puede garantizar que existe una sucesión $\left(a_{n}\right)$ en $A$ que converge a $x$. En el contexto más general de espacios topológicos, esto no siempre sucede.

1.2.1. Definición. Una sucesión $\left\{x_{n}: n \in \mathbb{N}\right\}$ en un espacio $X$ converge a $x \in X$ y se llama convergente, si para cada $U \in \tau(x, X)$, existe $N \in \mathbb{N}$ tal que $x_{n} \in U$ para $n>N$. Un espacio $X$ es Fréchet-Urysohn si para cada $A \subset X$ y cada $x \in \bar{A}$, existe una sucesión $\left\{x_{n}: n \in \mathbb{N}\right\} \subset A$ que converge a $x$.

1.2.2. Ejemplo. Supongamos que $X=\{0,1\}^{\omega_{1}}$ y $A=\left\{x \in X\right.$ : existe $F \subset \omega_{1}$ tal que $F$ es finito y $x(s)=0$ para $\left.s \in \omega_{1} \backslash F\right\}$; definamos $u \in\{0,1\}^{\omega_{1}}$ por la igualdad $u(s)=1$ para todo $s \in \omega_{1}$. Es fácil ver que $u \in \bar{A}$. Si $a_{n} \in A$ para $n \in \mathbb{N}$, hagamos $S=\left\{s \in \omega_{1}\right.$ : existe $a_{n} \in A$ para el cual $\left.a_{n}(s)=1\right\}$; es claro que $|S| \leq \omega$. Por tanto, si $t \in \omega_{1} \backslash S$ y $U=\{1\} \times\{0,1\}^{\omega_{1} \backslash\{t\}}$ se tiene que $U \in \tau(u, X)$; sin embargo, $a_{n} \notin U$ para todo $n \in \mathbb{N}$, así que $u \notin \overline{\left\{a_{n}: n \in \mathbb{N}\right\}}$ y por lo tanto la sucesión $\left\{a_{n}\right\}$ no converge a $u$.

Si $X$ es un espacio Fréchet-Urysohn, para cada $A \subset X$ que no es cerrado, existe una sucesión $\left\{a_{n}\right\}$ en $A$ que converge a un punto $x \in \bar{A} \backslash A$; lo recíproco no necesariamente se cumple.

1.2.3. Ejemplo. Sea $X=\{0\} \cup \bigcup_{i=1}^{\infty} X_{i}$, donde $X_{i}=\{1 / i\} \cup\left\{1 / i+1 /\left(i^{2}+k\right): k \in \omega\right\}$; se definirá $\tau(X)$ mediante una colección $\left\{\mathcal{B}_{x}: x \in X\right\}$, de bases locales. Si $x=1 / i+1 / j$ para alguna $j$, $\mathcal{B}_{x}=\{\{x\}\}$; si $x=1 / i$ para alguna $i$, se tiene que $\mathcal{B}_{x}=\left\{\{1 / i\} \cup\left\{1 / i+1 /\left(i^{2}+j+k\right): k \in \omega\right\}: j \in \omega\right\}$. Por último, $\mathcal{B}_{0}$ consiste de todos los conjuntos obtenidos de $X$ al quitar un número finito de conjuntos $X_{i}$, y un número finito de puntos de la forma $1 / i+1 / j$ de cada uno de los conjuntos restantes $X_{i}$; se checa fácilmente que $\left\{\mathcal{B}_{x}: x \in X\right\}$ es una colección de bases locales para una topología $T_{1}$ en $X$ y que cada elemento de $\mathcal{B}$ es un conjunto cerrado, de modo que el espacio resultante es de Tychonoff. Sea $A \subset X$ tal que $A \neq \bar{A}$; si $1 / i \in \bar{A} \backslash A$ para algún $i$, por definición de $\mathcal{B}_{1 / i}$ existe una sucesión creciente $\left\{j_{k}: k \in \mathbb{N}\right\} \subset \mathbb{N}$ para la cual $a_{k}=1 / i+1 /\left(i^{2}+j_{k}\right) \in A$ para todo $k$. La sucesión $\left\{a_{k}\right\}$ converge a $1 / i$. Si $1 / i \notin \bar{A} \backslash A$ para todo $i$, como $\{1 / i+1 / j\} \in \tau(X)$ para todo $j$, debe ser $0 \in \bar{A} \backslash A$; es fácil probar que existe una sucesión creciente $\left\{i_{k}: k \in \mathbb{N}\right\} \subset \mathbb{N}$ tal que $a_{k}=1 / i_{k} \in A$ para todo $k$. La sucesión $\left\{a_{k}\right\}$ converge a 0 . Así, se cumple la condición del párrafo anterior al ejemplo; sin embargo, $X$ no es Fréchet-Urysohn. Pues si $Y=X \backslash\{1 / i: i \in \mathbb{N}\}$, aunque $0 \in \bar{Y}$, no existe sucesión en $Y$ que converja a 0 .

El ejemplo anterior motiva la siguiente definición. 
1.2.4. Definición. Un espacio $X$ es secuencial si para cada $A \subset X$ tal que $A \neq \bar{A}$ existe una sucesión $\left\{x_{n}: n \in \mathbb{N}\right\} \subset A$ que converge a algún $x \in \bar{A} \backslash A$.

El espacio del ejemplo 1.2.3 es secuencial pero no Fréchet-Urysohn; en cambio el espacio en el ejemplo 1.2.2 no es Fréchet-Urysohn ni secuencial. Observamos también que los conceptos recién presentados involucran sucesiones; para el concepto de estrechez se prescinde de ellas y como se probará más adelante, es más general.

1.2.5. Definición. Sea $x \in X$; la estrechez de $x$ en $X$ es el cardinal $t(x, X)=\operatorname{mín}\{\kappa:$ si $x \in \bar{A}$, existe $B \subset A$ tal que $|B| \leq \kappa$ y $x \in \bar{B}\}$; la estrechez de $X$ es el cardinal $t(X)=\sup \{t(x, X): x \in X\}$. Un conjunto $A \subset X$ es $\kappa$-cerrado si para cada $B \subset A$ tal que $|B| \leq \kappa$ se cumple $\bar{B} \subset A$.

1.2.6. Ejemplo. Sea $X=\omega \cup\{\xi\} \subset \beta \omega$, donde $\beta \omega$ es la compactación de Čech-Stone de $\omega$ y $\xi \in \beta \omega \backslash \omega$; entonces $|X|=\omega$ y por ello $t(X) \leq \omega$. Como $\omega$ es denso en $\beta \omega$, el conjunto $\omega$ no es cerrado en $X$; por [Engelking, corolario 3.6.15], ninguna subsucesión de $\omega$, converge a $\xi$. Por tanto, $X$ no es secuencial.

Cabe mencionar que para el espacio $X=\{0,1\}^{\omega_{1}}$ del ejemplo 1.2.2, hemos demostrado que $u \in \bar{A}$ pero $u \notin \bar{B}$ para cualquier conjunto numerable $B \subset A$. De modo que $t(X)>\omega$.

1.2.7. Proposición. Para todo $X$, se tiene que $t(X) \leq \kappa$ si y sólo si para cualquier $A \subset X$ tal que $A \neq \bar{A}$, existe $B \subset A$ que cumple $|B| \leq \kappa$ y $\bar{B} \backslash A \neq \emptyset$.

Demostración. Necesidad. Supongamos que $A \subset X$ y $A \neq \bar{A}$, tomemos un punto $x \in \bar{A} \backslash A$. Como $t(X) \leq \kappa$, existe $B \subset A$ tal que $|B| \leq \kappa \mathrm{y} x \in \bar{B}$; es decir, existe $B \subset A$ que satisface las condiciones $|B| \leq \kappa$ y $\bar{B} \backslash A \neq \emptyset$.

Suficiencia. Si $\kappa \geq \omega, A \subset X$ y $C=\bigcup\{\bar{B}: B \subset A$ y $|B| \leq \kappa\}$, entonces $A \subset C \subset \bar{A}$, y si se prueba que $C$ es cerrado, se tendrá que $C=\bar{A}$. Por lo tanto, dado $x \in \bar{A}$, existirá $B \subset A$ tal que $|B| \leq \kappa$ y $x \in \bar{B}$; o sea $t(X) \leq \kappa$.

Así, sea $D \subset C$ tal que $|D| \leq \kappa$; luego existe $S$ con $|S| \leq \kappa$ para el que $D=\left\{x_{s}: x_{s} \in \bar{B}_{s}\right.$, $B_{s} \subset A$ y $\left|B_{s}\right| \leq \kappa$ para todo $\left.s \in S\right\}$. Si $E=\bigcup\left\{B_{s}: s \in S\right\}$, se obtiene $E \subset A$ y $|E| \leq \kappa$; se deduce que $\bar{E} \subset C$. Como $\bar{D}=\overline{\left\{x_{s}: s \in S\right\}} \subset \overline{\bigcup\left\{\bar{B}_{s}: s \in S\right\}} \subset \bar{E}$, se tiene que $\bar{D} \backslash C=\emptyset$; según la hipótesis, $C=\bar{C}$.

Si $\kappa<\omega$, como la condición nunca se cumple, se deduce que $t(X) \leq \kappa$.

1.2.8. Observaciones.(i) La proposición 1.2.7 también puede enunciarse como sigue: para todo $X$, se tiene que $t(X) \leq \kappa$ si y sólo si para todo $A \subset X$, si $A$ es $\kappa$-cerrado, entonces $A$ es cerrado.

(ii) Dado $A \subset X$, sea $[A]_{\kappa}=\bigcup\{\bar{B}: B \subset A$ y $|B| \leq \kappa\}$; de acuerdo con la definición 1.2 .5 se tiene que $A \subset X$ es $\kappa$-cerrado si y sólo si $A=[A]_{\kappa}$.

1.2.9. Teorema. Para todo $X$, si se cumple alguna propiedad de la lista también se cumple la siguiente:

(i) $\chi(X) \leq \omega$

(ii) $X$ es Fréchet-Urysohn. 
(iii) $X$ es secuencial.

(iv) $t(X) \leq \omega$.

Demostración. (i) $\Rightarrow$ (ii). Sean $x \in \bar{A}$ y $\left\{U_{i}: i \in \mathbb{N}\right\}$ una base local del espacio $X$ en $x$; si se elige $x_{i} \in A \cap U_{1} \cap \ldots \cap U_{i}$ para todo $i \in \mathbb{N}$, se obtiene una sucesión $\left\{x_{i}: i \in \mathbb{N}\right\}$ en $A$ que converge a $x$. De modo que $X$ es Fréchet-Urysohn.

(ii) $\Rightarrow$ (iii). Es consecuencia inmediata de las definiciones.

(iii) $\Rightarrow$ (iv). Se sigue de la proposición 1.2.7.

Ya se han dado ejemplos de espacios que muestran que las implicaciones inversas no se cumplen, excepto para la primera.

1.2.10. Ejemplo. Sea $X=\{p\} \cup \bigcup_{\alpha<\omega} X_{\alpha}$ el erizo de Urysohn con $\omega$ espinas (véase el ejemplo 1.1.8), donde $X_{\alpha}=\left\{x_{i}^{\alpha}: i \in \mathbb{N}\right\}$ para todo $\alpha$; ya se probó que $\chi(p, X)>\omega$, luego $\chi(X)>\omega$. Para ver que $X$ es Fréchet-Urysohn, tomemos cualesquiera $A \subset X$ y $x \in \bar{A}$; por definición de $\tau(X)$, si $x \neq p$, se tiene que $x \in A$, por lo cual se supondrá que $x=p$. Además, existe $\alpha<\omega$ tal que para todo $n \in \mathbb{N}$ existe $i_{n}>n$ para el cual $x_{i_{n}}^{\alpha} \in A$. Por tanto, para cada $V \in \tau(x, X)$ existe $N \in \mathbb{N}$ que satisface la condición $x_{i_{n}}^{\alpha} \in V$ para todo $n>N$; ya que $V$ es arbitrario, la sucesión $\left\{x_{i_{n}}^{\alpha}: n \in \mathbb{N}\right\} \subset A$ converge a $p$.

En el capítulo 2 se dará un ejemplo de un espacio $X$ para el cual $\psi(X) \leq \omega<t(X)$ (ejemplo 2.2.14); de aquí se deduce que no se puede sustituir la condición $\chi(X) \leq \omega$ por $\psi(X) \leq \omega$ en el teorema 1.2.9.

1.2.11. Proposición. Para todo $X$ y $x \in X$ se verifica $t(x, X) \leq \chi(x, X)$.

Demostración. Supongamos que $\chi(x, X) \leq \kappa \mathrm{y} x \in \bar{A}$; entonces existe una base local $\mathcal{B}$ de $X$ en $x$ para la cual $|\mathcal{B}| \leq \kappa$. Para cada $U \in \mathcal{B}$, elíjase $x_{U} \in A \cap U$ y sea $B=\left\{x_{U}: U \in \mathcal{B}\right\}$; luego $B \subset A,|B| \leq \kappa$ y por ser $\mathcal{B}$ una base en $x$ se deduce que cada $W \in \tau(x, X)$ contiene algún $x_{U}$. En consecuencia, $x \in \bar{B}$ y $t(x, X) \leq \kappa$; luego $t(x, X) \leq \chi(x, X)$.

1.2.12. Ejemplo. Sea $X$ el erizo de Urysohn con $\omega$ espinas; ya se vio en el ejemplo 1.1 .8 que $\chi(p, X)>\omega$. Puesto que $|X|=\omega$, se obtiene que $t(p, X) \leq \omega$; de manera que la desigualdad en la proposición 1.2.11 puede ser estricta.

1.2.13. Teorema. Si $X$ es linealmente ordenado, entonces $t(x, X)=\chi(x, X)$ para cada $x \in X$.

Demostración. Sea $\leq$ un orden lineal en $X$ que genera $\tau(X)$. Para $u, v \in X$ con $u<v$ hagamos $(\leftarrow, u)=\{x \in X: x<u\},(u, \rightarrow)=\{x \in X: x>u\}$ y $(u, v)=\{x \in X: u<x<v\} ;$ supongamos que $x \in X, t(x, X)=\kappa, U=(\leftarrow, x)$ y $V=(x, \rightarrow)$. Sea $\kappa \leq \omega$; por el teorema 1.1.11 y la proposición 1.2.11, es suficiente probar que $\psi(x, X) \leq \kappa$. Para ésto se consideran casos:

(a) Si $x \notin \bar{U} \cup \bar{V}$, el punto $x$ es aislado en $X$, luego $\psi(x, X)=1 \leq \kappa$.

(b) Si $x \in \bar{V} \backslash \bar{U}$, sea $A \subset V$ tal que $|A| \leq \kappa$ y $x \in \bar{A}$; entonces $\mathcal{B}=\{X \backslash \bar{U}\} \cup\{(\leftarrow, a): a \in A\}$ satisface las condiciones $\mathcal{B} \subset \tau(X)$ y $|\mathcal{B}| \leq \kappa$. Sea $y \in X \backslash\{x\}$; si $y \in \bar{U}$, se tiene que $y \notin \cap \mathcal{B}$. Si $y \in V$, entonces $W=(\leftarrow, y)$ cumple $W \in \tau(x, X)$ y existe $a \in A \cap W$, de modo que $y \notin(\leftarrow, a)$. Así, $y \notin \bigcap \mathcal{B}$, de donde $\bigcap \mathcal{B}=\{x\}$, por lo cual $\psi(x, X) \leq \kappa$. 
(c) Si $x \in \bar{U} \backslash \bar{V}$, se procede de manera similar a (b).

(d) Si $x \in \bar{U} \cap \bar{V}$, sean $A \subset U$ y $B \subset V$ tales que $x \in \bar{A} \cap \bar{B},|A| \leq \kappa$ y $|B| \leq \kappa$; la familia $\mathcal{B}=\{(a, b): a \in A$ y $b \in B\}$ cumple $\mathcal{B} \subset \tau(X)$ y $|\mathcal{B}| \leq \kappa$. Si $y<x$, como $(y, \rightarrow) \in \tau(x, X)$, existe $a \in A \cap(y, \rightarrow)$, así que $y \notin(a, b)$, para todo $b \in B$, de donde $y \notin \bigcap \mathcal{B}$. Si $y>x$, análogamente se obtiene $y \notin \bigcap \mathcal{B}$; por tanto, $\psi(x, X) \leq \kappa$.

Ahora si $\kappa<\omega$, entonces $x$ es un punto aislado y se cumple $t(x, X)=\chi(x, X)$.

1.2.14. Definición. Un espacio compacto se llama diádico si es la imagen continua de $\{0,1\}^{\kappa}$ para algún cardinal $\kappa$. Supongamos que $\eta=\left\{X_{t}: t \in T\right\}$ es una familia de espacios, $X$ es su producto topológico y $a \in X$ está fijo; dado $x \in X$, el conjunto $\operatorname{supp}(x)=\{t \in T: x(t) \neq a(t)\}$ se llama soporte de $x$. El $\Sigma$-producto de $\eta$ con punto básico $a$ es $\Sigma(a)=\{x \in X:|\operatorname{supp}(x)| \leq \omega\}$.

1.2.15. Proposición. Supongamos que $M_{t}$ es un espacio compacto con base numerable para todo $t \in T$ y $a \in M=\prod_{t \in T} M_{t}$; entonces $\Sigma(a)$ es un subespacio $\omega$-cerrado denso en $M$ tal que para cada conjunto numerable $A \subset \Sigma(a)$ se cumple $w\left(c_{M}(A)\right) \leq \omega$ y por lo tanto $c_{M}(A)$ es metrizable.

Demostración. Sea $U=\prod_{t \in T} U_{t} \neq \emptyset$ tal que $U_{t} \in \tau\left(M_{t}\right)$ para todo $t \in T$ y $S=\left\{t \in T: U_{t} \neq M_{t}\right\}$ es un conjunto finito; tómese $x_{t} \in U_{t}$ para todo $t \in S$ y hágase $x(t)=x_{t}$ si $t \in S$ y $x(t)=a_{t}$ si $t \in T \backslash S$. Es claro que $x \in U \cap \Sigma(a)$, así que $c l_{M}(\Sigma(a))=M$. Si $A \subset \Sigma(a)$ es numerable, puesto que $|\operatorname{supp}(x)| \leq \omega$ para todo $x \in \Sigma(a)$, se tiene que $E=\bigcup\{\operatorname{supp}(x): x \in A\}$ es un conjunto numerable; dado que el espacio $Y=\prod_{t \in T \backslash E}\{a(t)\} \times \prod_{t \in E} M_{t} \subset \Sigma(a)$ es homeomorfo a $\prod_{t \in E} M_{t}$, por [Engelking, teorema 4.2.8], se deduce que $Y$ es un subespacio compacto metrizable (y por ello cerrado) en $M$. Claramente $A \subset Y$; por tanto, $c l_{M}(A) \subset Y \subset \Sigma(a)$ es metrizable y $w\left(c l_{M}(A)\right) \leq \omega$.

1.2.16. Proposición. Sean $X$ un espacio compacto metrizable y $f: X \rightarrow Y$ una función continua sobreyectiva; entonces $Y$ es compacto y metrizable.

Demostración. Por [Engelking, teorema 4.2.8], se tiene que $w(X) \leq \omega$; se sabe que $Y$ es compacto y por [Engelking, teorema 3.1.22], se obtiene $w(Y) \leq w(X)$. De nuevo por [Engelking, teorema 4.2.8], se infiere que $Y$ es metrizable.

1.2.17. Teorema (Arhangel'skit). Supongamos que $D$ es un subespacio denso en $X=\prod_{t \in T} X_{t}$, donde $n w\left(X_{t}\right) \leq \omega$ para todo $t \in T$ y $f: D \rightarrow \mathbb{R}$ es una función continua; si la proyección sobre la cara $X_{T^{\prime}}$ se representa por $q_{T^{\prime}}: X \rightarrow X_{T^{\prime}}=\prod_{t \in T^{\prime}} X_{t}$ para todo $T^{\prime} \subset T$, entonces existe un conjunto numerable $S \subset T$ y una función continua $h: q_{S}(D) \rightarrow \mathbb{R}$ tales que $f=h \circ\left(q_{S} \mid D\right)$.

Demostración. Si $U=\prod_{t \in T} U_{t}$ es un conjunto de la base canónica de $X$, se escribirá core $(U)=$ $\left\{t \in T: U_{t} \neq X_{t}\right\}$. Para cada $y \in \mathbb{R}$ sea $\mathcal{B}_{y}$ una base local numerable de $\mathbb{R}$ en $y$; por ser $f$ continua, para cada $x \in D$ existe una familia numerable $\mathcal{U}_{x}$ de conjuntos de la base canónica de $X$ tal que $x \in \bigcap \mathcal{U}_{x}$ y para cada $O \in \mathcal{B}_{f(x)}$ existe $U \in \mathcal{U}_{x}$ que cumple $f(U \cap D) \subset O$. Por tanto, para cada $x \in D$, el conjunto $T_{x}=\bigcup\left\{\operatorname{core}(U): U \in \mathcal{U}_{x}\right\}$ es numerable. Sea $x_{0} \in D$ y hágase $A_{0}=\left\{x_{0}\right\}$ y $S_{0}=T_{x_{0}}$; supongamos que ya tenemos unos conjuntos numerables $A_{n} \subset D$ y $S_{n} \subset T$. Como $n w\left(X_{S_{n}}\right) \leq \omega$, el espacio $D_{S_{n}}=q_{S_{n}}(D) \subset X_{S_{n}}$ es separable, así que es posible hallar un conjunto numerable $A_{n+1} \subset D$ para el cual $A_{n} \subset A_{n+1}$ y el conjunto $q_{S_{n}}\left(A_{n+1}\right)$ sea denso en $D_{S_{n}}$; si se define $S_{n+1}=\bigcup\left\{T_{x}: x \in A_{n+1}\right\}$ se completa un proceso inductivo que nos permite formar los conjuntos $A=\bigcup\left\{A_{n}: n \in \omega\right\}$ y $S=\bigcup\left\{S_{n}: n \in \omega\right\}$. 
Se afirma que el conjunto $A_{S}=q_{S}(A)$ es denso en $D_{S}=q_{S}(D)$. Tómese $y \in D$ y hágase $W=\prod_{t \in S} W_{t} \in \tau\left(q_{S}(y), X_{S}\right)$ un conjunto básico canónico de $X_{S}$; puesto que $K=$ core $(W)$ es finito, existe $n \in \omega$ tal que $K \subset S_{n}$. Como $W^{\prime}=\prod_{t \in S_{n}} W_{t} \in \tau\left(q_{S_{n}}(y), X_{S_{n}}\right)$ y $q_{S_{n}}\left(A_{n+1}\right)$ es denso en $D_{S_{n}}$, se obtiene $W^{\prime} \cap q_{S_{n}}\left(A_{n+1}\right) \neq \emptyset$; luego existe $x \in A_{n+1}$ tal que $x(t) \in W_{t}$ para todo $t \in K$. Tenemos la igualdad $W_{t}=X_{t}$ para cada $t \in S \backslash K$, lo cual implica que $x(t) \in W_{t}$ para todo $t \in S$, así que $q_{S}(x) \in W \cap A_{S}$; por eso $q_{S}(y) \in c l_{D_{S}}\left(A_{S}\right)$. Ésto prueba la afirmación.

Ahora se probará lo siguiente:

(1) Si $U$ es un conjunto básico canónico en $X$ y $O$ es un conjunto abierto en $\mathbb{R}$ tal que $f(U \cap D) \subset$ $O$, entonces $f\left(q_{S}^{-1}\left(q_{S}(U)\right) \cap A\right) \subset \bar{O}$.

Supongamos que existe $w \in q_{S}^{-1}\left(q_{S}(U)\right) \cap A$ para el cual $f(w) \in \mathbb{R} \backslash \bar{O}$; entonces existe $O_{w} \in \mathcal{B}_{f(w)}$ que cumple $O_{w} \subset \mathbb{R} \backslash \bar{O}$. Tómese $W \in \mathcal{U}_{w}$ tal que $f(W \cap D) \subset O_{w}$; como core $(W) \subset S$, tenemos $q_{S}^{-1}\left(q_{S}(W)\right)=W$. Por lo tanto, $q_{S}(w) \in q_{S}(W) \cap q_{S}(U)$ y de aquí se obtiene $W \cap U \neq \emptyset$; dado que $D$ es denso en $X$ existe $d \in D \cap W \cap U$. En consecuencia, $f(d) \in f(D \cap W) \subset O_{w} \subset \mathbb{R} \backslash \bar{O}$; sin embargo, $f(d) \in f(D \cap U) \subset O$, lo cual es una contradicción que prueba (1).

Se afirma que si $x, y \in D$ y $q_{S}(x)=q_{S}(y)$ entonces $f(x)=f(y)$. Supongamos que $f(x) \neq f(y)$; entonces existen $O_{x} \in \tau(f(x), \mathbb{R})$ y $O_{y} \in \tau(f(y), \mathbb{R})$ tales que $\bar{O}_{x} \cap \bar{O}_{y}=\emptyset$. Elíjanse conjuntos $W_{x}$ y $W_{y}$ de la base canónica de $X$ que cumplan $x \in W_{x}, y \in W_{y}, f\left(D \cap W_{x}\right) \subset O_{x}$ y $f\left(D \cap W_{y}\right) \subset O_{y}$; puesto que $q_{S}$ transforma cada conjunto básico canónico en $X$ en un conjunto básico canónico en $X_{S}$, tenemos que $q_{S}$ es un mapeo abierto. Por tanto, $q_{S}\left(W_{x}\right)$ y $q_{S}\left(W_{y}\right)$ son abiertos en $X_{S}$ y $q_{S}(x)=q_{S}(y) \in q_{S}\left(W_{x}\right) \cap q_{S}\left(W_{y}\right) ;$ como $q_{S}(x)=q_{S}(y) \in D_{S}$ y $A_{S}$ es denso en $D_{S}$, existe $z \in A$ para el cual $q_{S}(z) \in q_{S}\left(W_{x}\right) \cap q_{S}\left(W_{y}\right)$. En consecuencia, $z \in q_{S}^{-1}\left(q_{S}\left(W_{x}\right)\right) \cap q_{S}^{-1}\left(q_{S}\left(W_{y}\right)\right) \cap A$; por la condición (1) se obtiene $f(z) \in \bar{O}_{x} \cap \bar{O}_{y}$, lo cual es una contradicción.

Para cada $y \in D_{S}$ elíjase $x \in q_{S}^{-1}(y)$ y hágase $h(y)=f(x)$; la afirmación del párrafo anterior muestra que esta definición de la función $h: D_{S} \rightarrow \mathbb{R}$ es consistente. Por definición de $h$, se tiene $f(x)=h\left(q_{S}(x)\right)$ para todo $x \in D$; se probará que $h$ es continua. Sea $y \in D_{S}$ y tómense $x \in q_{S}^{-1}(y)$ y $G \in \tau(h(y), \mathbb{R})$; elíjanse $O, H \in \tau(h(y), \mathbb{R})$ tales que $h(y) \in O \subset \bar{O} \subset H \subset \bar{H} \subset G$. Puesto que $h(y)=f(x)$ se obtiene $O \in \tau(f(x), \mathbb{R})$; por ser $f$ continua en $x$, existe un conjunto básico canónico $U \in \tau(x, X)$ para el cual $f(U \cap D) \subset O$. El conjunto $V=q_{S}(U)$ es abierto en $X_{S}$, además, $y \in V$. Sea $B=q_{S}^{-1}(V) \cap A$; por la condición (1), se cumple $f(B) \subset \bar{O}$.

Por construcción de $\mathcal{U}_{w}$ existe $U_{w} \in \mathcal{U}_{w}$ tal que $f\left(U_{w} \cap D\right) \subset H$ para todo $w \in B$. Sea $U(B)=$ $\bigcup\left\{U_{w}: w \in B\right\} ;$ como $\operatorname{core}\left(U_{w}\right) \subset S$ implica $q_{S}^{-1}\left(q_{S}\left(U_{w}\right)\right)=U_{w}$, se deduce que $q_{S}^{-1}\left(q_{S}(U(B))\right)=$ $U(B)$. Se mostrará que $q_{S}^{-1}(V) \cap D \subset c l_{X}(U(B))$; para ello, supóngase que el conjunto $C=$ $q_{S}^{-1}(V) \backslash c l_{X}(U(B))$ verifica $C \cap D \neq \emptyset$. Si $d \in C \cap D$, entonces $q_{S}(d) \in q_{S}(C) \in \tau\left(X_{S}\right) \mathrm{y}$ $q_{S}(C) \cap q_{S}(U(B))=\emptyset$; dado que $B \subset U(B)$, tenemos $q_{S}(B)=V \cap q_{S}(A) \subset q_{S}(U(B))$. Puesto que $q_{S}(C) \in \tau\left(q_{S}(d), X_{S}\right)$ y $q_{S}(d) \in c l_{D_{S}}\left(q_{S}(A)\right)$, obtenemos $q_{S}(C) \cap q_{S}(A) \neq \emptyset$; nótese que $q_{S}(C) \subset V$ y por ello $\emptyset \neq q_{S}(C) \cap q_{S}(A) \subset q_{S}(C) \cap V \cap q_{S}(A) \subset q_{S}(C) \cap q_{S}(U(B)) \neq \emptyset$. De la contradicción hallada se infiere que $q_{S}^{-1}(V) \cap D \subset c l_{X}(U(B))$.

Nótese que $f(U(B) \cap D)=\bigcup\left\{f\left(U_{w} \cap D\right): w \in B\right\} \subset H$ por definición de cada $U_{w}$; luego, $f\left(q_{S}^{-1}(V) \cap D\right) \subset f\left(c l_{X}(U(B)) \cap D\right)=f\left(c l_{D}(U(B) \cap D)\right) \subset \bar{H}$. Debido a que $f=h \circ\left(q_{S} \mid D\right)$, se sigue que $f\left(q_{S}^{-1}(V) \cap D\right)=h\left(V \cap D_{S}\right)$, por lo cual $h\left(V \cap D_{S}\right) \subset \bar{H} \subset G$; ésto indica que $h$ es continua en $y$, y por ello es continua en $D_{S}$. 
1.2.18. Proposición. Supongamos que $M_{t}$ es un espacio compacto con base numerable para todo $t \in T$ y $a \in M=\prod_{t \in T} M_{t}$; si $g: \Sigma(a) \rightarrow Z$ es una función continua sobre el espacio compacto $Z$, entonces $Z$ es metrizable.

Demostración. Por [Engelking, teorema 3.2.5], puede suponerse que $Z \subset \mathbb{I}^{\kappa}$ para $\mathbb{I}=[0,1]$ y algún cardinal $\kappa$; para cada $\alpha<\kappa$, sea $p_{\alpha}: \mathbb{I}^{\kappa} \rightarrow \mathbb{I}_{\alpha}=\mathbb{I}$ la correspondiente proyección natural. Puesto que $\Sigma(a)$ es denso en $M$ por la proposición 1.2.15, podemos aplicar el teorema 1.2.17 a $p_{\alpha} \circ g: \Sigma(a) \rightarrow \mathbb{I}$ para hallar un conjunto numerable $S_{\alpha} \subset T$ y una función continua $h_{\alpha}: q_{S_{\alpha}}(\Sigma(a)) \rightarrow \mathbb{I}$ tales que $p_{\alpha} \circ g=h_{\alpha} \circ\left(q_{S_{\alpha}} \mid \Sigma(a)\right)$; es claro que $q_{S_{\alpha}}(\Sigma(a))=M_{S_{\alpha}}=\prod_{t \in S_{\alpha}} M_{t}$, por lo cual $h_{\alpha}$ tiene dominio $M_{S_{\alpha}}$, de donde la función $G_{\alpha}=h_{\alpha} \circ q_{S_{\alpha}}$ tiene dominio $M$ y $G_{\alpha} \mid \Sigma(a)=p_{\alpha} \circ g$.

Defínase la función $f: M \rightarrow \mathbb{I}^{\kappa}$ mediante $f(x)(\alpha)=G_{\alpha}(x)$ para todo $x \in M$ y $\alpha<\kappa$; como $p_{\alpha} \circ f=G_{\alpha}$ es una función continua para todo $\alpha<\kappa$, la función $f$ es continua. Dado $x \in \Sigma(a)$ se tiene que $p_{\alpha}(f(x))=G_{\alpha}(x)=p_{\alpha}(g(x))$ para todo $\alpha<\kappa$, lo que implica que $f(x)=g(x)$; por lo tanto, $f(\Sigma(a))=g(\Sigma(a))=Z$, de donde $f(M) \subset c l_{\mathbb{I}^{\kappa}}(f(\Sigma(a)))=Z$.

A continuación se probará lo siguiente:

(1) Para cada conjunto numerable $P \subset Z$, el espacio $\bar{P}$ es compacto y metrizable.

Puesto que $f(\Sigma(a))=Z$, puede hallarse un conjunto numerable $A \subset \Sigma(a)$ tal que $P=f(A)$; por la proposición 1.2.15, se tiene que $F=c l_{M}(A) \subset \Sigma(a)$ es compacto y metrizable, así que por la proposición 1.2.16, se deduce que $f(F)$ es compacto y metrizable. Dado que $\bar{P} \subset f(F)$ es claro que se cumple (1).

Ahora, se probará la siguiente propiedad:

(2) Si $R \subset Z$ y $|R| \leq \omega_{1}$, entonces existe un conjunto numerable $P \subset Z$ tal que $R \subset \bar{P}$ y por lo tanto $w(R) \leq \omega$.

Tómese $A \subset \Sigma(a)$ que cumpla $|A| \leq \omega_{1}$ y $f(A)=R$; como $|\operatorname{supp}(x)| \leq \omega$ para todo $x \in A$, se obtiene que $|E| \leq \omega_{1}$ para el conjunto $E=\bigcup\{\operatorname{supp}(x): x \in A\}$. El espacio $M_{E}=\prod_{t \in E} M_{t}$ es homeomorfo a $Y=\left\{q_{T \backslash E}(a)\right\} \times M_{E}$, por lo cual $Y$ es un producto de $|E|$ espacios separables; por [Engelking, teorema 2.3.15], el espacio $Y$ es separable. Elíjase un conjunto denso numerable $B \subset Y$; dado que $A \subset Y$ se tiene que $R \subset f(Y) \subset \overline{f(B)}$. Como $f(B) \subset Z$ y $|f(B)| \leq \omega$, por (1), se obtiene que $\overline{f(B)}$ es compacto y metrizable; por [Engelking, teorema 4.2.8], se deduce que $w(\overline{f(B)}) \leq \omega \mathrm{y}$ de aquí $w(R) \leq \omega$, con lo cual queda probado (2).

Supongamos que $Z$ no es separable y tómese $z_{0} \in Z$; si $\beta<\omega_{1}$ y hemos construido un conjunto $C=\left\{z_{\alpha}: \alpha<\beta\right\} \subset Z$, como $|C| \leq \omega$, debe ser $\bar{C} \neq Z$, así que podemos elegir $z_{\beta} \in Z \backslash \bar{C}$. Este proceso inductivo puede realizarse para todo $\beta<\omega_{1}$, con lo cual se obtiene un conjunto $Y=\left\{z_{\alpha}: \alpha<\omega_{1}\right\} \subset Z$ tal que $z_{\beta} \notin \overline{\left\{z_{\alpha}: \alpha<\beta\right\}}$ para todo $\beta<\omega_{1}$. De (2) se sigue que $w(Y) \leq \omega$, luego $Y$ es separable; por ésto, $Y \subset \overline{\left\{z_{\alpha}: \alpha<\beta\right\}}$ para algún $\beta<\omega_{1}$, de donde $z_{\beta} \in \overline{\left\{z_{\alpha}: \alpha<\beta\right\}}$. La contradicción resultante muestra que $Z$ es separable. Sea $P \subset Z$ tal que $\bar{P}=Z$ y $|P| \leq \omega$; por (1), el espacio $Z$ es metrizable.

1.2.19. Teorema. Si $X$ es un espacio compacto diádico, entonces las siguientes condiciones son equivalentes:

(i) $\chi(X) \leq \omega$; 
(ii) $t(X) \leq \omega$;

(iii) $X$ es metrizable.

Demostración. (i) $\Rightarrow$ (ii). Se sigue del teorema 1.2.9.

(ii) $\Rightarrow$ (iii). Sea $M=\{0,1\}^{\kappa}$ tal que existe una función continua sobreyectiva $f: M \rightarrow X$; tómese $a \in M$ y hágase $Q=f(\Sigma(a))$. Sea $z \in X$ arbitrario; por la proposición 1.2 .15 y como $f$ es un mapeo cerrado ([Engelking, teorema 3.1.12]), se tiene que $\bar{Q}=X$. Puesto que $t(X) \leq \omega$, existe $R \subset Q$ que cumple $z \in \bar{R}$ y $|R| \leq \omega$. Elíjase un conjunto numerable $A \subset \Sigma(a)$ para el cual $R=f(A)$; por la misma proposición 1.2.15, el conjunto $F=c l_{M}(A) \subset \Sigma(a)$ es compacto y metrizable. De la proposición 1.2.16 se desprende que $f(F)$ es compacto y metrizable; puesto que $R \subset f(F)$ se deduce que $z \in \bar{R} \subset f(F) \subset Q$, así que $X=Q$. Por la proposición 1.2.18, se concluye que $X$ es metrizable.

(iii) $\Rightarrow($ i). Ésto se cumple para cualquier espacio métrico.

1.2.20. Teorema (Arhangel'skit). Para todo espacio compacto infinito $X$, se cumple $|X| \leq 2^{\chi(X)}$.

Demostración. Si $\kappa=\chi(X)$ es finito, entonces $X$ es discreto, lo cual contradice su compacidad, así que $\kappa \geq \omega$. Sea $\mathcal{B}_{x}$ una base local en $x$ tal que $\left|\mathcal{B}_{x}\right| \leq \kappa$ para todo $x \in X$. Por inducción se construirá una sucesión transfinita $\left\{F_{\alpha}: \alpha<\kappa^{+}\right\}$de subconjuntos cerrados de $X$ tal que para cada $\alpha<\kappa^{+}$:

(1) $\left|F_{\alpha}\right| \leq 2^{\kappa}$ y $F_{\beta} \subset F_{\alpha}$ para $\beta<\alpha$;

(2) para cada familia finita $\mathcal{U} \subset \bigcup\left\{\mathcal{B}_{x}: x \in \bigcup_{\beta<\alpha} F_{\beta}\right\}$, si $X \neq \bigcup \mathcal{U}$, entonces $F_{\alpha} \backslash \bigcup \mathcal{U} \neq \emptyset$.

Sean $x_{0} \in X$ y $F_{0}=\left\{x_{0}\right\}$; supongamos que $\alpha_{0}<\kappa^{+}$y ya se tienen los conjuntos $F_{\alpha}$ que satisfacen (1) y (2) para $\alpha<\alpha_{0}$. Sea $\mathcal{B}=\bigcup\left\{\mathcal{B}_{x}: x \in \bigcup_{\alpha<\alpha_{0}} F_{\alpha}\right\}$ y $\mathbb{B}=\{\mathcal{U} \subset \mathcal{B}: \mathcal{U}$ es finito y $X \neq \bigcup \mathcal{U}\}$; luego $|\mathcal{B}| \leq 2^{\kappa}$ y $|\mathbb{B}| \leq 2^{\kappa}$. Denótese por $B$ el conjunto obtenido al elegir para cada $\mathcal{U} \in \mathbb{B}$ un punto en $X \backslash \bigcup \mathcal{U}$ y sea $F_{\alpha_{0}}=\overline{B \cup \bigcup_{\alpha<\alpha_{0}} F_{\alpha}}$. Entonces $|B| \leq 2^{\kappa}$ y por la proposición 1.1.4, tenemos que $\left|F_{\alpha_{0}}\right| \leq 2^{\kappa}$, de modo que (1) y (2) se cumplen para $\alpha=\alpha_{0}$, lo que completa la inducción.

Sea $F=\bigcup_{\beta<\kappa^{+}} F_{\beta}$; veremos que $F=X$, de donde $|X| \leq 2^{\kappa}$ por (1). Por ser $\kappa^{+}$regular y por (1), para cada $A \subset F$ que cumple $|A| \leq \kappa$, existe $\alpha<\kappa^{+}$tal que $A \subset F_{\alpha}$; así que $\bar{A} \subset F_{\alpha} \subset F$. Por la proposición 1.2.11, se tiene que $t(X) \leq \kappa$; luego, por la proposición 1.2.7, se obtiene $F=\bar{F}$. Por tanto, $F$ es compacto. Supongamos que existe $y \in X \backslash F$; para cada $x \in F$, elíjase $U_{x} \in \mathcal{B}_{x}$ con $y \notin U_{x}$. Entonces existe una familia finita $\mathcal{U} \subset\left\{U_{x}: x \in F\right\}$ para la cual $F \subset \bigcup \mathcal{U}$ y un $\alpha<\kappa^{+}$ tales que $\mathcal{U} \subset \bigcup\left\{\mathcal{B}_{x}: x \in \bigcup_{\beta<\alpha} F_{\beta}\right\}$; así, $X \neq \bigcup \mathcal{U}$ y $F_{\alpha} \backslash \bigcup \mathcal{U}=\emptyset$, en contradicción con (2). 


\subsection{Propiedades categóricas: teoremas y ejemplos}

En esta sección se analizan las relaciones que las propiedades Fréchet-Urysohn, secuencialidad y estrechez numerable tienen con las operaciones de toma de subespacios, producto y paso a espacios cocientes; asimismo, se proporcionan caracterizaciones de las propiedades Fréchet-Urysohn, secuencialidad y carácter numerable en términos de espacios metrizables. Además se presenta otra relación entre las propiedades Fréchet-Urysohn y secuencialidad y se caracterizan los espacios compactos de estrechez numerable en términos de sucesiones libres.

1.3.1. Proposición. Si $X$ es Fréchet-Urysohn y $Y \subset X$, entonces $Y$ es Fréchet-Urysohn.

Demostración. Sean $A \subset Y$ y $x \in c l_{Y}(A)$; por la igualdad $c l_{Y}(A)=c l_{X}(A) \cap Y$, existe una sucesión $\left\{x_{n}\right\} \subset A$ que converge a $x$ (en $X$ y en $Y$ ). Por tanto, $Y$ es Fréchet-Urysohn.

En contraste con lo anterior, un espacio $X$ puede ser secuencial y algún $Y \subset X$ no serlo; más adelante, en esta sección se caracterizan todos los espacios $X$ en los que se presenta esta situación (corolario 1.3.16). No obstante, se cumple lo siguiente:

1.3.2. Proposición. Si $X$ es secuencial y $Y \subset X$ es abierto o cerrado, entonces $Y$ es secuencial.

Demostración. Dado un conjunto cerrado $Y \subset X$ tomemos cualquier $A \subset Y$ tal que $c l_{Y}(A) \neq A$; entonces $A$ no es cerrado en $X$. Por lo tanto, existe una sucesión $\left\{x_{n}\right\}$ en $A$ que converge a algún $x \in X \backslash A$; como $Y$ es cerrado, $x \in Y$. Luego, $Y$ es secuencial. Ahora, supongamos que $Y \subset X$ es abierto y $A \subset Y$ cumple $c l_{Y}(A) \neq A$; así, existe $x \in c l_{Y}(A) \backslash A$. Sea $U \in \tau(x, X)$ tal que $\bar{U} \subset Y$; entonces $A \cap \bar{U}$ no es cerrado en $\bar{U}$. Ya hemos probado que la secuencialidad se hereda a subespacios cerrados, así que existe $\left\{x_{n}\right\} \subset A \cap \bar{U}$ que converge a cierto $p \in \bar{U} \backslash(A \cap \bar{U})$, de donde $p \in Y \backslash A$, lo que implica que $Y$ es secuencial.

1.3.3. Proposición. Para todo espacio $X$, si $Y \subset X$, entonces $t(Y) \leq t(X)$.

Demostración. Supongamos que $t(X) \leq \kappa \mathrm{y} A \subset Y$ cumple $y \in c l_{Y}(A)$; entonces $y \in c l_{X}(A)$ y por ello existe $B \subset A$ tal que $|B| \leq \kappa$ y $y \in c l_{X}(B)$. Es claro que $y \in c l_{Y}(B)$; por tanto, $t(Y) \leq \kappa$.

El siguiente ejemplo muestra que la propiedad de Fréchet-Urysohn no es multiplicativa; el mismo ejemplo sirve para ver que la secuencialidad y la estrechez numerable tampoco lo son.

1.3.4. Ejemplo. Existen espacios de Fréchet-Urysohn $X$ y $Y$ para los cuales $t(X \times Y)>\omega$. Sean $X=\{p\} \cup\left\{x_{n}^{m}: m, n \in \omega\right\}$ y $Y=\{q\} \cup\left\{y_{n}^{\alpha}: \alpha \in 2^{\omega}, n \in \omega\right\}$ los erizos de Urysohn con $\omega$ y $2^{\omega}$ espinas respectivamente (ejemplo 1.1.8); ya se probó en el ejemplo 1.2.10 que $X$ es Fréchet-Urysohn y de la misma manera se muestra que $Y$ es Fréchet-Urysohn. Para demostrar que $t(X \times Y)>\omega$ se hallará un conjunto $E \subset(X \backslash\{p\}) \times(Y \backslash\{q\})$ tal que $x=(p, q) \in \bar{E}$, pero $x \notin \bar{Q}$ para todo conjunto numerable $Q \subset E$. Con este propósito se empleará la siguiente notación: $\omega^{\kappa}$ es el conjunto de todas las funciones $f: \kappa \rightarrow \omega$; para cada $f \in \omega^{\omega}$ y cada $\phi \in \omega^{2^{\omega}}$ se escribirá $V_{f}=\{p\} \cup\left\{x_{n}^{m}: n \geq f(m)\right.$, $m \in \omega\}$ y $W_{\phi}=\{q\} \cup\left\{y_{n}^{\alpha}: n \geq \phi(\alpha), \alpha \in 2^{\omega}\right\}$. Si $f, g \in \omega^{\omega}$, por $f<^{*} g$ se entenderá que existe $k<\omega$ tal que $f(n)<g(n)$ para todo $n \geq k$. Es claro que $\left\{V_{f}: f \in \omega^{\omega}\right\}$ es una base local de $X$ en $p, \mathrm{y}\left\{W_{\phi}: \phi \in \omega^{2^{\omega}}\right\}$ es una base local de $Y$ en $q$.

Obsérvese que si $A \subset \omega^{\omega}$ es un conjunto numerable, entonces existe $f \in \omega^{\omega}$ tal que $g<^{*} f$ para todo $g \in A$, pues si $A=\left\{g_{k}: k \in \omega\right\}$, se define $f(n)=\max \left\{g_{k}(n): k \leq n\right\}+1$ para todo $n$, 


\section{Capítulo 1. Espacios Topológicos Generales}

con lo cual se obtiene $g_{k}<^{*} f$ para todo $k \in \omega$. Como $\left|\omega^{\omega}\right|=2^{\omega}$, se puede tomar una numeración $\left\{f_{\alpha}: \alpha \in 2^{\omega}\right\}$ del conjunto $\omega^{\omega}$.

Si $\alpha \in 2^{\omega}$, se tiene que $S_{\alpha}=\left\{\left(x_{f_{\alpha}(m)}^{m}, y_{m}^{\alpha}\right): m \in \omega\right\} \subset(X \backslash\{p\}) \times(Y \backslash\{q\})$; sea $E=\bigcup\left\{S_{\alpha}\right.$ : $\left.\alpha \in 2^{\omega}\right\}$. Para probar que $x \in \bar{E}$, tómese $U \in \tau(x, X \times Y)$; existen funciones $f \in \omega^{\omega}$ y $\phi \in \omega^{2^{\omega}}$ tales que $x \in V_{f} \times W_{\phi} \subset U$. Puesto que $f=f_{\alpha}$ para alguna $\alpha \in 2^{\omega}$, si $k=\phi(\alpha)$, se obtiene $\left(x_{f_{\alpha}(k)}^{k}, y_{k}^{\alpha}\right) \in S_{\alpha} \cap\left(V_{f} \times W_{\phi}\right)$; por lo tanto, $E \cap\left(V_{f} \times W_{\phi}\right) \neq \emptyset$, así que $E \cap U \neq \emptyset$, por lo cual $x \in \bar{E}$.

Para demostrar que $x \notin \bar{Q}$ para todo conjunto numerable $Q \subset E$, basta mostrar que $x \notin \overline{\bigcup_{\alpha \in P} S_{\alpha}}$ para todo conjunto numerable $P \subset 2^{\omega}$; tómese un tal conjunto $P$. Por la primera observación del párrafo anterior, existe $f \in \omega^{\omega}$ que cumple $f_{\alpha}<^{*} f$ para todo $\alpha \in P$; ahora, defínase $\phi \in \omega^{2^{\omega}}$ como sigue: si $\alpha \notin P$, hágase $\phi(\alpha)=0$. Si $\alpha \in P$, existe $k \in \omega$ tal que $f_{\alpha}(n)<f(n)$ para todo $n \geq k$; hágase $\phi(\alpha)=k$. Sea $z \in S_{\alpha}$ para alguna $\alpha \in P$; existe $m \in \omega$ para el cual $z=\left(x_{f_{\alpha}(m)}^{m}, y_{m}^{\alpha}\right)$. Si $x_{f_{\alpha}(m)}^{m} \in V_{f}$, se deduce que $f_{\alpha}(m) \geq f(m)$; por definición de $\phi$ se tiene $\phi(\alpha)=k>m$, de donde $y_{m}^{\alpha} \notin W_{\phi}$. En consecuencia, $z \notin V_{f} \times W_{\phi}$, de modo que $S_{\alpha} \cap\left(V_{f} \times W_{\phi}\right)=\emptyset$; dado que $\alpha \in P$ es arbitraria, $x \notin \overline{\bigcup_{\alpha \in P} S_{\alpha}}$.

Sin embargo, si nos restringimos a espacios compactos, entonces la estrechez numerable es numerablemente productiva y la secuencialidad es finitamente multiplicativa, como lo muestran los enunciados 1.3.6 y 1.3.18.

1.3.5. Lema. Si $f: X \rightarrow Y$ es un mapeo cerrado y $A \subset X$ es un conjunto $\kappa$-cerrado, entonces $B=f(A)$ es $\kappa$-cerrado.

Demostración. Supongamos que $D \subset B$ y que $|D| \leq \kappa$; para cada $d \in D$ elíjase $x_{d} \in A$ tal que $f\left(x_{d}\right)=d$ y defínase $E=\left\{x_{d}: d \in D\right\}$. Por tanto, $|E| \leq \kappa$, de donde $\bar{E} \subset A$; por ser $f$ cerrado, $\bar{D}=\overline{f(E)}=f(\bar{E}) \subset f(A)=B$. En consecuencia, $B$ es $\kappa$-cerrado.

1.3.6. Proposición. Si $X_{n}$ es compacto y $t\left(X_{n}\right) \leq \omega$ para $n \in \mathbb{N}$, entonces $t\left(\prod_{i=1}^{\infty} X_{i}\right) \leq \omega$.

Demostración. Primero se probará que si $X, Y$ son compactos, $t(X) \leq \omega$ y $t(Y) \leq \omega$, entonces $t(X \times Y) \leq \omega$; para ello considérese un conjunto $\omega$-cerrado $H \subset X \times Y$. Recurriendo a la observación 1.2.8(i), se mostrará que $H$ es cerrado; para ello basta tomar $(p, q) \in \bar{H}$ y ver que $(p, q) \in H$. Así, por ser $\{p\} \times Y$ cerrado en $X \times Y$ y $H$ un conjunto $\omega$-cerrado, de la definición se sigue que $T=H \cap(\{p\} \times Y)$ es $\omega$-cerrado en $X \times Y$ y en $\{p\} \times Y$; puesto que $t(\{p\} \times Y)=t(Y) \leq \omega$, según la observación 1.2.8(i), tenemos que $T=c l_{\{p\} \times Y}(T)=\bar{T}$, y es suficiente probar que $q \in \pi_{Y}(T)$, donde $\pi_{Y}: X \times Y \rightarrow Y$ es la proyección sobre $Y$. Supongamos que $q \notin \pi_{Y}(T)$; puesto que $X \times Y$ es compacto y $Y$ es $T_{2}$, vemos que $\pi_{Y}$ es cerrado y por ello $\pi_{Y}(T)$ es cerrado. Por ello, la cerradura $V$ de alguna vecindad de $q$ en $Y$ cumple $V \cap \pi_{Y}(T)=\emptyset$; dado que $(p, q) \in \operatorname{Int}(X \times V)$, se infiere que $(p, q) \in \overline{(X \times V) \cap H}$.

Tal como antes se deduce que por ser $X \times V$ cerrado en $X \times Y$ y $H$ un conjunto $\omega$-cerrado, el conjunto $(X \times V) \cap H$ es $\omega$-cerrado en $X \times Y$ y que la proyección $\pi_{X}: X \times Y \rightarrow X$ es cerrada; por el lema 1.3.5, se deduce que $S=\pi_{X}((X \times V) \cap H)$ es $\omega$-cerrado en $X$. Dado que $t(X) \leq \omega$, por la observación 1.2.8(i), se tiene que $S$ es cerrado; sin embargo, por la continuidad de $\pi_{X}$ tenemos que $p=\pi_{X}(p, q) \in \pi_{X}(\overline{(X \times V) \cap H}) \subset \bar{S}=S=\pi_{X}((X \times V) \cap H)$. Por lo tanto, existe $r \in V$ que cumple $(p, r) \in H$, lo que contradice que $(\{p\} \times V) \cap H=(\{p\} \times V) \cap T \subset \pi_{Y}^{-1}\left(V \cap \pi_{Y}(T)\right)=\emptyset$; 
consecuentemente $t(X \times Y) \leq \omega$. Si $[\mathbb{N}]^{<\omega}$ denota la familia de subconjuntos finitos de $\mathbb{N}$, por inducción se obtiene que $t\left(\prod_{i \in F} X_{i}\right) \leq \omega$ para cada $F \in[\mathbb{N}]^{<\omega}$.

Para el caso general, sean $X=\prod_{i=1}^{\infty} X_{i}$ y $A$ un subconjunto $\omega$-cerrado de $X$; tómense $p \in \bar{A}$ y $F \in[\mathbb{N}]^{<\omega}$. Por lo anterior, para el espacio $X_{F}=\prod_{i \in F} X_{i}$ se cumple $t\left(X_{F}\right) \leq \omega$; como la proyección $\pi_{F}: X \rightarrow X_{F}$ es cerrada, vemos que $\pi_{F}(A)$ es $\omega$-cerrado y por lo tanto cerrado en $X_{F}$. En consecuencia, $\pi_{F}(p) \in \pi_{F}(A)$; por tanto, existe $q_{F} \in A$ tal que $p\left|F=q_{F}\right| F$. Sea $B=\left\{q_{F}: F \in[\mathbb{N}]^{<\omega}\right\}$; entonces $|B| \leq \omega$ y $p \in \bar{B}$. Como $B \subset A$ y $A$ es un conjunto $\omega$-cerrado, $\bar{B} \subset A$; luego, $p \in A$. Por la observación 1.2.8(i), se concluye que $t(X) \leq \omega$.

Para caracterizar algunas propiedades de convergencia en términos de espacios metrizables, se emplearán mapeos abiertos, cocientes y pseudoabiertos.

1.3.7. Teorema. Para todo $Y$, se cumple $\chi(Y) \leq \omega$, si y sólo si existe un espacio metrizable $X$ y un mapeo abierto sobreyectivo $f: X \rightarrow Y$.

Demostración. Suficiencia. Se trata del corolario 1.1.3.

Necesidad. Sea $T$ el conjunto $\tau(Y)$ con la topología discreta; entonces, $T$ es metrizable. Por [Engelking, teoremas 4.1.3 y 4.2.2], tenemos que $T^{\omega}$ es metrizable; sea $X=\left\{x \in T^{\omega}:\{x(n): n \in \omega\}\right.$ es una base local en algún $y \in Y\}$. Dado $x \in X$, sea $f(x) \in Y$ el punto en el que $\{x(n): n \in \omega\}$ es una base local; por ser $Y$ un espacio $T_{1}$, la función $f$ está bien definida.

Dado $y \in Y$ tómese una base local $\left\{U_{n}: n \in \omega\right\}$ en $y$ y sea $x(n)=U_{n}$ para todo $n$; entonces $x \in X$ y $f(x)=y$, así que $f$ es suprayectiva. Para probar que $f$ es continua, si $x \in X$ y $f(x)=y \in U \in \tau(Y)$, existe $n \in \omega$ tal que $x(n) \subset U$; el conjunto $V=\{z \in X: z(n)=x(n)\}$ cumple $V \in \tau(x, X)$. Para cada $z \in V$ tenemos $f(z) \in \bigcap\{z(k): k \in \omega\} \subset z(n)=x(n) \subset U$, de donde $f(V) \subset U$; luego, $f$ es continua.

Para probar que $f(O) \in \tau(Y)$ para cada $O \in \tau(X)$ es suficiente mostrar que $x \in O$ implica $f(x) \in \operatorname{Int}(f(O))$. Sea $U=\prod_{i \in \omega} U_{i} \in \tau\left(T^{\omega}\right)$ tal que $x \in U \subset O$ y $U_{i}=T$ excepto para $i$ en cierto conjunto finito $F \subset \omega$; si se hace $n=\max F$, vemos que $W=\{z \in X: z(i)=x(i)$ para $i \leq n\} \subset O$. Como $V=x(0) \cap \ldots \cap x(n) \in \tau(X)$, basta probar que $V \subset f(O)$, en cuyo caso, $f(x) \in V \subset \operatorname{Int}(f(O))$. Sea $y \in V$ y elíjase una base local $\left\{V_{i}: i \in \omega\right\}$ en $y$ de manera que $V_{i}=x(i)$ para todo $i \leq n$; si $z(k)=V_{k}$ para todo $k \in \omega$, se obtiene que $z \in W \subset O$ y $f(z)=y$, así que $V \subset f(O)$.

1.3.8. Proposición. Si $f: X \rightarrow Y$ es un mapeo cociente y el espacio $X$ es secuencial, entonces $Y$ es secuencial.

Demostración. Por ser $f$ cociente, si $A \subset Y$ no es cerrado, $B=f^{-1}(A)$ tampoco lo es; por ello, existe una sucesión $\left\{x_{n}\right\} \subset B$ que converge a cierto $x \in \bar{B} \backslash B$. Puesto que $\left\{f\left(x_{n}\right)\right\} \subset A$ y converge a $f(x) \in \bar{A} \backslash A$, se infiere que $Y$ es secuencial.

1.3.9. Proposición. Si $f: X \rightarrow Y$ es un mapeo cociente, se cumple $t(Y) \leq t(X)$.

Demostración. Sean $t(X) \leq \kappa$ y $A \subset Y$ que no es cerrado; como $f$ es cociente, $A_{0}=f^{-1}(A)$ no es cerrado en $X$. Existe $B_{0} \subset A_{0}$ tal que $\left|B_{0}\right| \leq \kappa$ y $\bar{B}_{0} \backslash A_{0} \neq \emptyset$; si $B=f\left(B_{0}\right)$, entonces $B \subset A$, $|B| \leq \kappa$ y $\bar{B} \backslash A \neq \emptyset$. Por la proposición 1.2.7, tenemos que $t(Y) \leq \kappa$. 
Las proposiciones 1.3.8 y 1.3.9 muestran que la secuencialidad y la estrechez numerable son invariantes bajo mapeos cocientes; para la propiedad de Fréchet-Urysohn, ésto ya no sucede (ejemplo 1.3.19).

1.3.10. Definición. Un mapeo continuo sobreyectivo $f: X \rightarrow Y$ es pseudoabierto si para cada $y \in Y$ y cada $U \in \tau(X)$ tal que $f^{-1}(y) \subset U$, se cumple $y \in \operatorname{Int}(f(U))$. Un mapeo $f: X \rightarrow Y$ es hereditariamente cociente si para cada $Z \subset Y$ la restricción $f \mid f^{-1}(Z): f^{-1}(Z) \rightarrow Z$ es cociente.

1.3.11. Proposición. Un mapeo $f: X \rightarrow Y$ es pseudoabierto si y sólo si es hereditariamente cociente.

Demostración. Necesidad. Tomemos cualquier $Z \subset Y$; sean $T=f^{-1}(Z)$ y $f_{T}=f \mid T$. Para probar que $f_{T}$ es cociente, tómese $W \subset Z$ tal que $U=f_{T}^{-1}(W) \in \tau(T)$; entonces existe $V \in \tau(X)$ que cumple $U=V \cap T$, y si $z \in W$, tenemos $f^{-1}(z)=f_{T}^{-1}(z) \subset V$. Por ser $f$ pseudoabierta, $z \in O=\operatorname{Int}_{Y}(f(V))$; como $V \cap f^{-1}(Z \backslash W)=\emptyset$, se obtiene $z \in Q=O \cap Z \subset W$, así que $W \in \tau(Z)$, por lo cual $f_{T}$ es cociente.

Suficiencia. Tomemos cualesquiera $y \in Y$ y $U \in \tau(X)$ tales que $f^{-1}(y) \subset U$; se desea probar que $y \in \operatorname{Int}_{Y}(f(U))=Y \backslash \overline{Y \backslash f(U)}$. Supongamos lo contrario y sea $Z=(Y \backslash f(U)) \cup\{y\}$; entonces $g=f \mid f^{-1}(Z): f^{-1}(Z) \rightarrow Z$ es cociente y $Y \backslash f(U)$ no es cerrado en $Z$. Luego $g^{-1}(Y \backslash f(U))=f^{-1}(Y \backslash f(U))$ no es cerrado en $f^{-1}(Z)$; por ello $f^{-1}(y) \cap \overline{f^{-1}(Y \backslash f(U))} \neq \emptyset$, lo que contradice que $f^{-1}(y) \subset U \subset X \backslash f^{-1}(Y \backslash f(U))$.

1.3.12. Proposición. Si $f: X \rightarrow Y$ es pseudoabierto y $X$ es Fréchet-Urysohn, entonces $Y$ también lo es.

Demostración. Sean $A \subset Y$ y $y \in \bar{A}$; se puede suponer que $y \notin A$, de modo que $A$ no es cerrado en $Z=A \cup\{y\}$. Por la proposición 1.3.11, el mapeo $f \mid f^{-1}(Z): f^{-1}(Z) \rightarrow Z$ es cociente; por tanto, $f^{-1}(A)$ no es cerrado en $f^{-1}(Z)$. Luego, existe $x \in f^{-1}(y) \cap \overline{f^{-1}(A)}$; como $X$ es Fréchet-Urysohn, existe una sucesión $\left\{x_{n}\right\}$ en $f^{-1}(A)$ que converge a $x$. La sucesión $\left\{f\left(x_{n}\right)\right\}$ está contenida en $A$ y converge a $f(x)=y$. Esto prueba que $Y$ es Fréchet-Urysohn.

1.3.13. Teorema. El espacio $X$ es Fréchet-Urysohn si y sólo si $X$ es imagen pseudoabierta de un espacio metrizable.

Demostración. Suficiencia. Por el teorema 1.2.9, cada espacio metrizable es Fréchet-Urysohn; por la proposición 1.3.12, la imagen pseudoabierta de un espacio metrizable es Fréchet-Urysohn.

Necesidad. El espacio $M=\bigoplus\{S: S \subset X$ es una sucesión convergente, incluido el punto al que converge $\}$ es metrizable (véase [Engelking, teorema 4.2.1]). Sea $\phi: M \rightarrow X$ dada por $\phi(x)=x$ para todo $x \in M$; se probará que $\phi$ es pseudoabierta. Dado $x \in X$, hagamos $x_{n}=x$ para todo $n \in \omega$; entonces la sucesión $S=\left\{x_{n}: n \in \omega\right\}$ es un subespacio de $M$ y $x \in \phi(S)$, así que $\phi$ es sobreyectiva.

Para ver que $\phi$ es continua, tómense un cerrado $F \subset X$ y $z \in M \backslash \phi^{-1}(F)$; entonces existe un sumando $S \subset M$ con $z \in S$, así que $S \in \tau(M)$. Si $z$ es un punto aislado de $S$, se obtiene $\{z\} \in \tau(M)$ y $\{z\} \subset M \backslash \phi^{-1}(F)$. Si $z$ es un punto de acumulación de $S$, entonces $A=S \cap \phi^{-1}(F)$ es finito, pues en caso contrario, $\phi(S) \cap F$ es una subsucesión de $\phi(S)$ que converge a $\phi(z)$. Por 
tanto, $\phi(z) \in \bar{F} \backslash F$, lo cual es una contradicción; luego $S \backslash A \in \tau(x, M)$ y $S \backslash A \subset M \backslash \phi^{-1}(F)$, por lo cual $\phi^{-1}(F)$ es cerrado en $M$ y $\phi$ es continua.

Para probar que $\phi$ es pseudoabierta, tómense $s_{0} \in X$ y un conjunto $U \in \tau\left(\phi^{-1}\left(s_{0}\right), M\right)$; supongamos que $s_{0} \notin \operatorname{Int}_{X}(\phi(U))$. Por tanto, $s_{0} \in \overline{X \backslash \phi(U)}$; razón por la cual existe una sucesión $\left\{s_{n}: n \in \mathbb{N}\right\} \subset X \backslash \phi(U)$ que converge a $s_{0}$. Luego, la sucesión convergente $S=\left\{s_{n}: n \in \omega\right\}$ es un sumando en $M$. Sin embargo, $s_{0} \in U$ y para $n \in \mathbb{N}$, se tiene que $s_{n} \notin \phi(U)$, de donde $s_{n} \notin U$; ésto contradice que $\left\{s_{n}: n \in \mathbb{N}\right\}$ converge a $s_{0}$. Así que $s_{0} \in \operatorname{Int}_{X}(\phi(U))$ y $\phi$ es pseudoabierta.

1.3.14. Lema. (i) Si $X$ es secuencial, entonces $X$ es un espacio $k$.

(ii) Si $X$ es un espacio $k$ hereditariamente, $A \subset X$ y $x \in \bar{A} \backslash A$, entonces existe un compacto $Z \subset A \cup\{x\}$ tal que $x \in \overline{Z \backslash\{x\}}$.

Demostración. (i) Sea $A \subset X$ tal que $A \neq \bar{A}$; existe una sucesión $\left\{x_{n}: n \in \mathbb{N}\right\}$ en $A$ que converge a cierto $x_{0} \notin A$. En el subespacio $Z=\left\{x_{n}: n \in \omega\right\}$ de $X$, cada punto $x_{n}$ con $n \in \mathbb{N}$ es aislado y $x_{0}$ es su punto de acumulación; de modo que $Z$ es compacto y $A \cap Z$ no es cerrado en $Z$; por tanto $X$ es un espacio $k$.

(ii) El conjunto $A$ no es cerrado en $B=A \cup\{x\}$; puesto que $B$ es un espacio k, existe un compacto $Z \subset B$ para el cual $A \cap Z$ no es cerrado en $B$. Como $Z$ sí es cerrado en $B$, se deduce que $x \in \operatorname{cl}_{B}(Z \backslash\{x\}) \subset \overline{Z \backslash\{x\}}$.

1.3.15. Teorema. Las siguientes condiciones son equivalentes:

(i) X es Fréchet-Urysohn.

(ii) $X$ es hereditariamente secuencial.

(iii) $X$ es hereditariamente $k$.

Demostración. (i) $\Rightarrow$ (ii). Es consecuencia del teorema 1.2.9 y la proposición 1.3.1.

(ii) $\Rightarrow$ (iii). Se deduce del lema 1.3.14(i).

(iii) $\Rightarrow$ (i). Supongamos que $A \subset X$ y $x \in \bar{A} \backslash A$; existe un conjunto $S$ de cardinalidad mínima $\lambda$, tal que $S \subset A$ y $x \in \bar{S}$. Por tanto, $x \notin S$ y $\lambda \geq \omega$; por el lema 1.3.14, existe un compacto $Z \subset S \cup\{x\}$ para el cual $x \in \overline{Z \backslash\{x\}}$. Por la minimalidad de $\lambda$ y por la proposición 1.2.11 tenemos que $\lambda \leq t(x, Z) \leq \chi(x, Z)$; por el corolario 1.1 .12 se obtiene $\chi(x, Z) \leq \chi(Z) \leq|Z| \leq|S|=\lambda$, de donde $\lambda=\chi(x, Z)$. Sea $\left\{V_{\alpha}: \alpha<\lambda\right\}$ una base de $Z$ en $x$; por inducción se definirán unas sucesiones transfinitas $\left\{U_{\alpha}: \alpha<\lambda\right\}$ y $\left\{x_{\alpha}: \alpha<\lambda\right\}$ donde $U_{\alpha} \in \tau(x, Z)$ y $x_{\alpha} \in Z$, para cada $\alpha<\lambda$. La construcción garantizará que $\left\{x_{\beta}: \beta<\alpha\right\} \cap \bar{U}_{\alpha}=\emptyset, U_{\alpha} \subset V_{\alpha}$ y $x_{\alpha} \in\left(\bigcap_{\beta \leq \alpha} U_{\beta}\right) \backslash\{x\}$ para $\alpha<\lambda$. Por ser $Z$ regular, existe $U_{0} \in \tau(x, Z)$ tal que $\bar{U}_{0} \subset V_{0}$; elíjase $x_{0} \in U_{0} \backslash\{x\}$. Ahora, sea $\alpha<\lambda$ y supongamos que para todo $\beta<\alpha$ ya se han definido $U_{\beta}$ y $x_{\beta}$ que satisfacen las condiciones mencionadas; por la minimalidad de $\lambda$, tenemos que $x \notin \overline{\left\{x_{\beta}: \beta<\alpha\right\}}$. Luego existe $U_{\alpha} \in \tau(x, Z)$ que cumple $\overline{\left\{x_{\beta}: \beta<\alpha\right\}} \cap \bar{U}_{\alpha}=\emptyset$ y $\bar{U}_{\alpha} \subset V_{\alpha}$; puesto que $\lambda=\chi(x, Z)$, por el teorema 1.1 .10 , se cumple $\lambda=\psi(x, Z)$, así que existe $x_{\alpha} \in\left(\bigcap_{\beta \leq \alpha} U_{\beta}\right) \backslash\{x\}$. Esto completa la inducción.

Si $T=\left\{x_{\alpha}: \alpha<\lambda\right\} \cup\{x\}$, entonces $x$ es un punto de acumulación del conjunto $T$. Observe que $X_{\alpha}=X \backslash\left(\overline{\left\{x_{\beta}: \beta<\alpha\right\}} \cup \bar{U}_{\alpha+1}\right) \in \tau\left(x_{\alpha}, X\right)$ y $x_{\beta} \notin X_{\alpha}$ para cada $\alpha<\lambda$ y para $\beta \neq \alpha$; de modo que cada $x_{\alpha}$ es un punto aislado en $T$. Por ser $T$ hereditariamente $k$, del lema 1.3.14, se sigue que 
existe un conjunto compacto $K \subset T$ tal que $x \in \overline{K \backslash\{x\}}$; dado que $K \backslash\{x\} \subset Z \backslash\{x\} \subset S \subset A$, por la minimalidad de $\lambda$, se obtiene $\lambda \leq|K \backslash\{x\}|=|K|$. Elíjase en $K \backslash\{x\}$ un conjunto infinito numerable $R$; por ser $K$ compacto, $R$ tiene un punto de acumulación $z$ en $K$ y sólo puede ser $z=x$. Puesto que $R \subset A$, se infiere que $R$ es una sucesión convergente y $X$ es Fréchet-Urysohn.

1.3.16. Corolario. Si $X$ es un espacio secuencial, entonces $X$ contiene un subespacio que no es secuencial si y sólo si $X$ no es Fréchet-Urysohn.

Demostración. Es consecuencia inmediata del teorema 1.3.15.

1.3.17. Teorema. Un espacio $X$ es secuencial, si y sólo si $X$ es la imagen cociente de un espacio metrizable.

Demostración. Suficiencia. Supongamos que $M$ es espacio metrizable y $X$ es su imagen cociente; entonces $\chi(M) \leq \omega$. Por el teorema 1.2.9, se obtiene que $M$ es secuencial y por la proposición 1.3.8, se concluye que $X$ es secuencial.

Necesidad. Sea $M=\bigoplus\{S: S \subset X$ es una sucesión convergente, incluido el punto al que converge $\}$. Consideremos la función $\phi: M \rightarrow X$ dada por $\phi(x)=x$ para todo $x \in M$; tal como en la demostración del teorema 1.3 .13 se prueba que $M$ es metrizable y que $\phi$ es sobreyectiva y continua. Para demostrar que $\phi$ es cociente tomemos cualquier $P \subset X$ tal que $\bar{P} \neq P$; como $X$ es secuencial, existe una sucesión $\left\{s_{n}: n \in \mathbb{N}\right\} \subset P$ que converge a cierto $s_{0} \in \bar{P} \backslash P$. Por tanto, el sumando $S=\left\{s_{n}: n \in \omega\right\} \subset M$ es una sucesión convergente; luego $s_{0} \in \overline{\phi^{-1}(P)} \backslash \phi^{-1}(P)$, es decir, $\phi^{-1}(P)$ no es cerrado y por eso $\phi$ es cociente.

La siguiente proposición se puede generalizar al caso en el que $X$ y $Y$ son espacios secuenciales y $X$ es localmente secuencialmente compacto; este caso generaliza también aquellos en los que $X$ y $Y$ son secuenciales y $X$ es localmente compacto o es numerablemente compacto.

1.3.18. Proposición. Si $X$ y $Y$ son espacios secuenciales y $X$ es compacto, entonces $X \times Y$ es secuencial.

Demostración. Por el teorema 1.3.17, existen espacios metrizables $X_{0}, Y_{0}$ y mapeos cocientes $f: X_{0} \rightarrow X, g: Y_{0} \rightarrow Y$. Por el teorema 1.2.9 y el lema 1.3.14(i), el espacio $X_{0}$ es $k$; por [Engelking, teorema 3.3.27], el espacio $X \times Y$ también es $k$. Por [Engelking, teorema 3.3.28], la función $f \times g: X_{0} \times Y_{0} \rightarrow X \times Y$ es cociente. Aplicando el teorema 1.3.17 una vez más, concluimos que $X \times Y$ es secuencial.

1.3.19. Ejemplo. Sea $Y$ el espacio del ejemplo 1.2.3; puesto que $Y$ es secuencial, por el teorema 1.3.17, existe un espacio $X$ metrizable y un mapeo cociente $f: X \rightarrow Y$. Por el teorema 1.2.9, se tiene que $X$ es Fréchet-Urysohn; pero a pesar de que $f$ es cociente, $Y$ no es Fréchet-Urysohn.

1.3.20. Definición. Un subconjunto $\left\{x_{\alpha}: \alpha<\kappa\right\}$ de un espacio $X$ es una sucesión libre de longitud $\kappa$, si $\overline{\left\{x_{\alpha}: \alpha<\lambda\right\}} \cap \overline{\left\{x_{\alpha}: \alpha \geq \lambda\right\}}=\emptyset$ para todo $\lambda<\kappa$.

Para el siguiente enunciado, si $A \subset X$, se denotará por $[A]^{\omega}$ al conjunto $\{x \in X$ : si $H$ es un conjunto $G_{\delta}$ en $X$ y $x \in H$, entonces $\left.H \cap A \neq \emptyset\right\}$; además, se usará el significado de $[A]_{\omega}$ introducido en la observación 1.2.8(ii). 
1.3.21. Lema. Para todo espacio compacto $X$, se cumple $\left[[A]_{\omega}\right]^{\omega}=\bar{A}$ para cualquier $A \subset X$.

Demostración. Como es claro que $\left[[A]_{\omega}\right]^{\omega} \subset \bar{A}$, queda por probar que $\bar{A} \subset D=\left[[A]_{\omega}\right]^{\omega}$. Supongamos que existe $x \in \bar{A} \backslash D$; entonces existe $\mathcal{A}=\left\{U_{n}: n \in \mathbb{N}\right\} \subset \tau(X)$ tal que $x \in H=\bigcap \mathcal{A} \subset X \backslash[A]_{\omega}$. Sean $V_{1}=U_{1}$, y $V_{n} \in \tau(x, X)$ tales que $\bar{V}_{n} \subset V_{n-1} \cap U_{n}$ para $n>1$; así, $F=\bigcap_{n=1}^{\infty} V_{n}=\bigcap_{n=1}^{\infty} \bar{V}_{n}$ es un $G_{\delta}$ cerrado para el cual $x \in F \subset H$. Según el teorema 1.1.10, existe una base externa $\mathcal{D}$ de $F$ en $X$ que cumple $|\mathcal{D}| \leq \omega$; puesto que $x \in F \cap \bar{A}$, para cada $W \in \mathcal{D}$ puede elegirse $x_{W} \in W \cap A$. El conjunto $B=\left\{x_{W}: W \in \mathcal{D}\right\}$ satiface las condiciones $|B| \leq \omega$ y $B \subset A$, por lo cual $F \cap \bar{B} \subset H \cap[A]_{\omega}=\emptyset$. Puesto que $\mathcal{D}$ es una base externa de $F$ en $X$, existe $W \in \mathcal{D}$ para el cual $W \cap \bar{B}=\emptyset$, lo que contradice que $x_{W} \in W \cap B$.

1.3.22. Teorema (Arhangel'skit). Para todo espacio compacto $X$, se verifica $t(X) \leq \omega$ si y sólo si $X$ no tiene sucesiones libres no numerables.

Demostración. Necesidad. Supongamos que $t(X) \leq \omega$ y existe una sucesión libre $S=\left\{x_{\alpha}: \alpha<\omega_{1}\right\}$ en $X$. Si $F_{\beta}=\overline{\left\{x_{\alpha}: \alpha \geq \beta\right\}}$, entonces $\mathcal{F}=\left\{F_{\beta}: \beta<\omega_{1}\right\}$ es una familia decreciente de conjuntos cerrados en $X$; como $X$ es compacto, existe $y \in \bigcap \mathcal{F}$. Por tanto, $y \in \bar{S}$; si $A \subset S$ cumple $|A| \leq \omega$, entonces $A \subset\left\{x_{\alpha}: \alpha<\beta\right\}$ para algún $\beta<\omega_{1}$, así que $\bar{A} \cap F_{\beta}=\emptyset$, de donde $y \notin \bar{A}$. Ésto contradice la desigualdad $t(X) \leq \omega$. En consecuencia, $X$ no tiene sucesiones libres no numerables.

Suficiencia. Supongamos que $X$ no tiene sucesiones libres no numerables y $t(X)>\omega$; por la observación 1.2.8(i), existe un conjunto $\omega$-cerrado $A \subset X$ que no es cerrado. Por el lema 1.3.21 y la observación 1.2.8(ii), se obtiene que $\bar{A}=[A]^{\omega}$, así que existe $x \in[A]^{\omega} \backslash A$; por ello se cumple:

(1) $H \cap A \neq \emptyset$ si $H$ es un conjunto $G_{\delta}$ que contiene a $x$.

Sean $a_{0} \in A$ y $H_{0}=X$; supongamos que $\alpha<\omega_{1}$ y se han hallado puntos $\left\{a_{\beta}: \beta<\alpha\right\} \subset A$ y una familia de conjuntos $G_{\delta}$ cerrados $\left\{H_{\beta}: \beta<\alpha\right\}$ con las siguientes propiedades:

(2) $\left\{x, a_{\beta}\right\} \subset H_{\beta}$ para todo $\beta<\alpha$;

(3) $H_{\beta} \subset H_{\gamma}$ para $\gamma<\beta<\alpha$;

(4) $\overline{\left\{a_{\gamma}: \gamma<\beta\right\}} \cap H_{\beta}=\emptyset$ para todo $\beta<\alpha$.

Puesto que $A$ es un conjunto $\omega$-cerrado y $x \notin A$, se sigue que $x \notin P=\overline{\left\{a_{\gamma}: \gamma<\alpha\right\}}$; sean $V_{1}=X \backslash P$ y $V_{n} \in \tau(x, X)$ tales que $\bar{V}_{n} \subset V_{n-1}$ para $n>1$. De esta forma, $H=\bigcap_{n=1}^{\infty} V_{n}=\bigcap_{n=1}^{\infty} \bar{V}_{n}$ es un $G_{\delta}$ cerrado para el cual $x \in H \subset X \backslash P$. Si $H_{\alpha}=H \cap \bigcap\left\{H_{\beta}: \beta<\alpha\right\}$ por (1), existe $a_{\alpha} \in H_{\alpha} \cap A$. Observamos que las condiciones (2)-(4) se cumplen también para $\beta=\alpha$, así que la inducción proporciona un conjunto $S=\left\{a_{\alpha}: \alpha<\omega_{1}\right\}$. Ahora, si $\beta<\omega_{1}$, por (2) y (3), se obtiene que $\left\{a_{\gamma}: \gamma \geq \beta\right\} \subset H_{\beta}$; por (4) y por ser $H_{\beta}$ cerrado, $\overline{\left\{a_{\gamma}: \gamma<\beta\right\}} \cap \overline{\left\{a_{\gamma}: \gamma \geq \beta\right\}} \subset$ $\overline{\left\{a_{\gamma}: \gamma<\beta\right\}} \cap H_{\beta}=\emptyset$. Ésto prueba que $S$ es una sucesión libre de longitud no numerable, lo cual es una contradicción; por tanto, $t(X) \leq \omega$. 


\section{CAPÍTULO 2}

\section{Propiedades de convergencia en espacios de funciones}

Este capítulo constituye la parte medular de la tesis, pues en él se estudia el comportamiento de las mismas propiedades de convergencia consideradas en el capítulo 1, pero ahora en ciertos espacios de funciones en presencia de estructuras algebraicas; para ello, primero se definen los espacios de funciones y la topología que en ellos se considerará. Después se define la estructura algebraica y su relación con la topología, para así obtener una operación $\mathcal{C}$ entre espacios topológicos; se analiza la relación entre $\mathcal{C}$ y las operaciones topológicas producto y suma de espacios, toma de subespacios y el paso a imágenes continuas. También se observa la manera como cambian algunas propiedades topológicas al aplicarse la operación $\mathcal{C}$.

\subsection{Restricciones que implica la estructura algebraica de $C_{p}(X)$}

En esta sección primero se definen los espacios de funciones con los que se trabajará, que son espacios de funciones continuas con la topología producto; después se definen las estructuras algebraicas en los espacios mencionados, que son la de grupo y anillo inducidas por $\mathbb{R}$ en ellos, para luego obtener de su interrelación las estructuras de grupo y anillo topológicos. Esto determina una riqueza estructural en la que no sólo las propiedades de convergencia, sino también otras, cambian drásticamente según se mostrará.

Se recuerda que en un producto cartesiano de espacios se toma por defecto la topología producto y que $\mathbb{R}, \mathbb{Q}$ y $\mathbb{N}$ se considerarán con su topología usual si no se indica otra cosa.

2.1.1. Definición. Mediante $Y^{X}$ (respectivamente $C(X, Y)$ ) se representa al conjunto de todas las funciones (resp. continuas) de $X$ a $Y$ mientras $C(X)$ representa a $C(X, \mathbb{R})$. Por $C_{p}(X, Y)$ se hace referencia al espacio topológico obtenido de $C(X, Y)$ como subespacio de $Y^{X}$; en particular, $C_{p}(X)$ es $C_{p}(X, \mathbb{R})$. Dados $x_{1}, \ldots, x_{n} \in X$ y $U_{1}, \ldots, U_{n} \in \tau(Y)$, se denota a $\left\{f \in C(X, Y): f\left(x_{i}\right) \in U_{i}\right.$ para todo $i \leq n\}$ por medio de $O\left(x_{1}, \ldots, x_{n} ; U_{1}, \ldots, U_{n}\right)$ u $O\left(x_{1}, \ldots, x_{n} ; U_{1}, \ldots, U_{n}\right)_{(X, Y)}$ para indicar cuáles son los espacios $X, Y$ involucrados; además, si tomamos $f \in C(X)$ y $\epsilon>0$, se denota al conjunto $O\left(x_{1}, \ldots, x_{n} ;\left(f\left(x_{1}\right)-\epsilon, f\left(x_{1}\right)+\epsilon\right), \ldots,\left(f\left(x_{n}\right)-\epsilon, f\left(x_{n}\right)+\epsilon\right)\right)=\left\{g \in C(X):\left|g\left(x_{i}\right)-f\left(x_{i}\right)\right|<\right.$ $\epsilon$ para todo $i \leq n\}$ por $O\left(f ; x_{1}, \ldots, x_{n} ; \epsilon\right)$ u $O\left(f ; x_{1}, \ldots, x_{n} ; \epsilon\right)_{(X)}$ para indicar cuál es el espacio $X$ 
involucrado.

2.1.2. Proposición. Para cualquier base $\mathcal{U}$ de $Y$, la familia $\mathcal{B}=\left\{O\left(x_{1}, \ldots, x_{n} ; U_{1}, \ldots, U_{n}\right)\right.$ : $x_{i} \in X, U_{i} \in \mathcal{U}, i=1, \ldots, n$ y $\left.n \in \mathbb{N}\right\}$ es una base de $C_{p}(X, Y)$; dada $f \in C_{p}(X)$, la familia $\mathcal{B}_{f}=\left\{O\left(f ; x_{1}, \ldots, x_{n} ; \epsilon\right): x_{i} \in X, i=1, \ldots, n, n \in \mathbb{N} y \epsilon>0\right\}$ es una base local de $C_{p}(X)$ en $f$.

Demostración. Supongamos que $\mathcal{U}$ es una base de $Y$ y $\tau$ es la topología del espacio $C_{p}(X, Y)$. Dado $O=O\left(x_{1}, \ldots, x_{n} ; U_{1}, \ldots, U_{n}\right) \in \mathcal{B}$ se puede suponer que $x_{i} \neq x_{j}$ para $i \neq j$, tomando intersecciones de conjuntos $U_{i}$ en caso necesario; hágase $Y_{x}=U_{i}$ si $x=x_{i}$ para alguna $i \leq n$, y $Y_{x}=Y$ si $x \neq x_{i}$ para todo $i$. De este modo, $O=\prod_{x \in X} Y_{x} \cap C(X, Y) \in \tau$; por tanto, $\mathcal{B} \subset \tau$. Ahora, si $f \in V \in \tau$, existe un conjunto básico canónico $B$ en $Y^{X}$ tal que $f \in W=B \cap C(X, Y) \subset V$; sean $B=\prod_{x \in X} Y_{x}$ y $\left\{x_{1}, \ldots, x_{n}\right\}$ el subconjunto de $X$ en el que $Y_{x} \neq Y$. Para cada $i \leq n$ existe $U_{i} \in \mathcal{U}$ tal que $f\left(x_{i}\right) \in U_{i} \subset Y_{x_{i}}$, así que $f \in O=O\left(x_{1}, \ldots, x_{n} ; U_{1}, \ldots, U_{n}\right) \subset W$ donde $O \in \mathcal{B}$. De lo anterior, por una parte se deduce que $\mathcal{B}$ es una base de $C_{p}(X, Y)$; por otra parte, si en particular $Y=\mathbb{R}$ y $U_{i}=\left(f\left(x_{i}\right)-\epsilon, f\left(x_{i}\right)+\epsilon\right)$ para todo $i \leq n$ y cierta $\epsilon>0$, se obtiene que $\mathcal{B}_{f}$ es una base local de $C_{p}(X)$ en $f$.

2.1.3. Proposición. El espacio $C_{p}(X)$ es denso en $\mathbb{R}^{X}$.

Demostración. Sean $f \in \mathbb{R}^{X}$ y $U$ un conjunto básico canónico en $\mathbb{R}^{X}$ que contiene a $f$; entonces existen $x_{1}, \ldots, x_{n} \in X$ tales que $U=\prod_{x \in X} \mathbb{R}_{x}$, en donde $\mathbb{R}_{x}=\mathbb{R}$ si $x \neq x_{i}$ para todo $i \leq n$. Por ser $X$ espacio de Tychonoff, $X_{i}=\bigcup_{j \neq i}\left\{x_{j}\right\}$ es cerrado para todo $i$ y existe $g_{i} \in C_{p}(X)$ tal que $g_{i}\left(x_{i}\right)=1$ y $g_{i}\left(X_{i}\right) \subset\{0\}$ para todo $i \leq n$; por tanto, $g=\sum_{i=1}^{n} f\left(x_{i}\right) g_{i} \in C_{p}(X)$ y cumple $g\left(x_{i}\right)=f\left(x_{i}\right)$ para todo $i \leq n$. En consecuencia $g \in U$, lo cual prueba que $\overline{C_{p}(X)}=\mathbb{R}^{X}$.

Las operaciones en $\mathbb{R}$ inducen de manera natural las correspondientes operaciones en $C_{p}(X)$, de suma $f+g$ donde $(f+g)(x)=f(x)+g(x)$ para todo $x \in X$ y de producto $f g$ donde $(f g)(x)=f(x) g(x)$ para todo $x \in X$, que le dan la estructura de anillo. Más aún, las operaciones se interrelacionan de manera especial con la topología de $C_{p}(X)$.

2.1.4. Proposición. Para todo espacio $X$, la función $S: C_{p}(X) \times C_{p}(X) \rightarrow C_{p}(X)$ dada por $S(f, g)=f+g$ para toda $(f, g) \in C_{p}(X) \times C_{p}(X)$, es continua.

Demostración. Tómense las funciones $f, g \in C_{p}(X)$ y la vecindad $O=O\left(f+g ; x_{1}, \ldots, x_{n} ; \epsilon\right)$ de $f+g$ arbitrarias; si $U=O\left(f ; x_{1}, \ldots, x_{n} ; \epsilon / 2\right)$ y $V=O\left(g ; x_{1}, \ldots, x_{n} ; \epsilon / 2\right)$, tómese $h \in U$ y $k \in V$. Así, $\left|(f+g)\left(x_{i}\right)-(h+k)\left(x_{i}\right)\right|<\frac{\epsilon}{2}+\frac{\epsilon}{2}=\epsilon$ para todo $i \leq n$; por tanto, $h+k \in O$. De modo que $S(U \times V) \subset O$; por la proposición 2.1.2, se tiene que $S$ es continua.

Obsérvese que según la proposición 2.1.4, dada $g \in C_{p}(X)$, la función $T: C_{p}(X) \rightarrow C_{p}(X)$ definida por $T(f)=f+g$ es continua; por tanto, su inversa que se calcula como $T^{-1}(f)=f-g$ para toda $f \in C_{p}(X)$, también es continua. Consecuentemente $T$ es un homeomorfismo.

2.1.5. Proposición. Para todo espacio $X$, la función $P: C_{p}(X) \times C_{p}(X) \rightarrow C_{p}(X)$ dada por $P(f, g)=f g$ para toda $(f, g) \in C_{p}(X) \times C_{p}(X)$, es continua.

Demostración. Sean $f, g \in C_{p}(X)$ y $O=O\left(f g ; x_{1}, \ldots, x_{n} ; \epsilon\right)$ arbitrarios; si dada $j \leq n$ se hace $O_{j}(f)=O\left(f ; x_{j} ; \min \left\{1, \frac{\epsilon}{2\left(\left|g\left(x_{j}\right)\right|+1\right)}\right\}\right)$ y $O_{j}(g)=O\left(g ; x_{j} ; \frac{\epsilon}{2\left(\left|f\left(x_{j}\right)\right|+1\right)}\right)$, entonces $P\left(O_{j}(f) \times O_{j}(g)\right) \subset$ 
$O\left(f g ; x_{j} ; \epsilon\right)$. Para verlo, obsérvese que si $h \in O_{j}(f)$ y $k \in O_{j}(g)$, entonces $\left|(f g)\left(x_{j}\right)-(h k)\left(x_{j}\right)\right| \leq$ $\left|f\left(x_{j}\right)-h\left(x_{j}\right)\right|\left|g\left(x_{j}\right)\right|+\left|h\left(x_{j}\right)\right|\left|g\left(x_{j}\right)-k\left(x_{j}\right)\right|<\epsilon / 2+\epsilon / 2=\epsilon$; es decir, $h k \in O\left(f g ; x_{j} ; \epsilon\right)$ para todo $j \leq n$. Por tanto, $P\left(\bigcap_{j=1}^{n} O_{j}(f) \times O_{j}(g)\right) \subset \bigcap_{j=1}^{n} P\left(O_{j}(f) \times O_{j}(g)\right) \subset \bigcap_{j=1}^{n} O\left(f g ; x_{j} ; \epsilon\right)=O$; de la proposición 2.1.2 se sigue que $P$ es continua.

De este modo $C_{p}(X)$ con la operación suma es grupo topológico y con ambas operaciones es anillo topológico. Estas operaciones junto con las peculiaridades de la topología de $C_{p}(X)$, limitan drásticamente en los espacios $C_{p}(X)$ las posibilidades de ciertas propiedades topológicas de los espacios generales (proposiciones 2.1.7 y 2.1.8), pero a la vez enriquecen notablemente las relaciones entre estas propiedades.

Así, recordando que la celularidad de un espacio $X$ es $c(X)=\sup \{|\mathcal{U}|: \mathcal{U} \subset \tau(X) \backslash\{\emptyset\}$ es una familia disjunta\}, y que una familia $\mathcal{A}$ de subconjuntos de $X$ es localmente finita si para cada $x \in X$, existe $U \in \tau(x, X)$ tal que $|\{A \in \mathcal{A}: U \cap A \neq \emptyset\}|<\omega$, tenemos lo siguiente.

2.1.6. Lema. Para todo espacio $X$, si $Y$ es denso en $X$, entonces $c(X)=c(Y)$.

Demostración. Sea $\gamma \subset \tau(X) \backslash\{\emptyset\}$ una familia disjunta; entonces $\mu=\{U \cap Y: U \in \gamma\} \subset \tau(Y) \backslash\{\emptyset\}$ es una familia disjunta y $|\mu|=|\gamma|$. Por tanto, $c(X) \leq c(Y)$. Ahora, sea $\mu \subset \tau(Y) \backslash\{\emptyset\}$ una familia disjunta; para cada $U \in \mu$ elíjase $V_{U} \in \tau(X)$ tal que $V_{U} \cap Y=U$. Si $U, U^{\prime} \in \mu$ son distintos, entonces $V_{U} \cap V_{U^{\prime}} \cap Y=U \cap U^{\prime}=\emptyset$; como $\bar{Y}=X$, tenemos que $V_{U} \cap V_{U}^{\prime}=\emptyset$ y por lo tanto la familia $\gamma=\left\{V_{U}: U \in \mu\right\} \subset \tau(X) \backslash\{\emptyset\}$ es disjunta. Puesto que $|\mu|=|\gamma|$, se obtiene que $c(Y) \leq c(X)$.

2.1.7. Proposición. Para todo espacio $X$, se cumple $c\left(C_{p}(X)\right) \leq \omega$.

Demostración. Puesto que $\mathbb{R}$ es un espacio separable, por [Engelking, corolario 2.3.18], se cumple $c\left(\mathbb{R}^{X}\right) \leq \omega$; por la proposición 2.1 .3 , se deduce que $\mathbb{R}^{X}=\overline{C_{p}(X)}$, así que por el lema 2.1.6, se obtiene $c\left(C_{p}(X)\right) \leq \omega$.

2.1.8. Proposición. Para todo espacio $X$, si $\gamma \subset \tau\left(C_{p}(X)\right)$ es una familia localmente finita, entonces $|\gamma| \leq \omega$.

Demostración. Supongamos que $\gamma \subset \tau\left(C_{p}(X)\right) \backslash\{\emptyset\}$ es una familia localmente finita y que cada uno de los miembros de una familia disjunta maximal $\mu \subset \tau\left(C_{p}(X)\right) \backslash\{\emptyset\}$ intersecta un número finito de elementos de $\gamma$; entonces $\overline{\cup \mu}=C_{p}(X)$. Puesto que $c\left(C_{p}(X)\right) \leq \omega$, por la proposición 2.1.7 se deduce que $|\mu| \leq \omega$; para cada $U \in \gamma$ existe $V \in \mu$ tal que $U \cap V \neq \emptyset$. Si $\gamma$ no fuera numerable, existiría $U \in \mu$ para la cual $|\{V \in \gamma: U \cap V \neq \emptyset\}|>\omega$, lo cual es una contradicción; por tanto, $|\gamma| \leq \omega$

Con respecto a las propiedades de convergencia, podrá añadirse que para todo $X$, se verifica $\chi\left(C_{p}(X)\right) \leq \omega$ si y sólo si $C_{p}(X)$ es metrizable; esto ya se podría obtener como una consecuencia del teorema 1.1.13, sin embargo, se deducirá en la siguiente sección, a partir de una herramienta de la $C_{p}$-teoría (corolario 2.2.3).

Como ya se ha mencionado, el paso de $X$ a $C_{p}(X)$, o de $(X, Y)$ a $C_{p}(X, Y)$ puede considerarse como una operación $\mathcal{C}$ entre espacios topológicos; aquí se entiende por operación una función de $\mathbb{E}$ en $\mathbb{E}$ o de $\mathbb{E} \times \mathbb{E}$ en $\mathbb{E}$, donde $\mathbb{E}$ es la clase de los espacios de Tychonoff. En vista de la gran diferencia que puede existir entre $X$ y $C_{p}(X)$, según se ha mostrado, podríamos preguntarnos si hay 
propiedades que se conserven bajo $\mathcal{C}$. Primero observamos que si $\mathcal{P}$ es una propiedad topológica productiva y hereditaria, al tenerla $\mathbb{R}$ la tendrá $C_{p}(X)$, pues $C_{p}(X) \subset \mathbb{R}^{X}$; así se deduce que $C_{p}(X)$ es un espacio de Tychonoff para todo $X$, luego, $\mathcal{C}$ es efectivamente una operación.

Podemos considerar la relación entre $\mathcal{C}$ y las operaciones producto y suma de una familia de espacios; si $X$ es un espacio discreto, $C_{p}(X)$ resulta ser un producto, $C_{p}(X)=\mathbb{R}^{X}$; para un espacio arbitrario $X$ se cumplen los enunciados 2.1 .10 y 2.1.11.

2.1.9. Lema. Supongamos que $w \in C_{p}(Y, Z)$ y $h_{w}: C_{p}(X, Y) \rightarrow C_{p}(X, Z)$ está dado por $h_{w}(f)=$ $w \circ f$ para toda $f \in C_{p}(X, Y)$; entonces $h_{w}$ es continuo.

Demostración. Tómense $f \in C_{p}(X, Y)$ y $U \in \tau\left(C_{p}(X, Z)\right)$ tales que $g=h_{w}(f) \in U$; entonces existe $V=O\left(x_{1}, \ldots, x_{n} ; U_{1}, \ldots, U_{n}\right) \in \tau\left(C_{p}(X, Z)\right)$ para el cual $g \in V \subset U$. Luego, el conjunto $W=$ $O\left(x_{1}, \ldots, x_{n} ; w^{-1}\left(U_{1}\right), \ldots, w^{-1}\left(U_{n}\right)\right) \in \tau\left(C_{p}(X, Y)\right)$ satisface las condiciones $f \in W$ y $h_{w}(W) \subset V$; por lo tanto, $h_{w}$ es continuo.

2.1.10. Proposición. Dada una familia de espacios $\left\{Y_{t}: t \in T\right\}$, para cualquier espacio $X$ el espacio $C_{p}\left(X, \prod_{t \in T} Y_{t}\right)$ es homeomorfo a $\prod\left\{C_{p}\left(X, Y_{t}\right): t \in T\right\}$.

Demostración. Se empleará la siguiente notación: $Y=\prod_{t \in T} Y_{t}$ y $C=\prod_{t \in T} C_{p}\left(X, Y_{t}\right)$ mientras se escribirá $p_{t}: Y \rightarrow Y_{t}$ y $q_{t}: C \rightarrow C_{p}\left(X, Y_{t}\right)$ para las respectivas proyecciones naturales; asimismo dado $x \in X$, el mapeo $r_{x}: Y^{X} \rightarrow Y_{x}$ donde $Y_{x}=Y$ será la proyección natural, y si $t \in T$, por $\pi_{t, x}$ se denotará a la restricción a $C_{p}\left(X, Y_{t}\right)$ de la proyección natural de $Y_{t}^{X}$ sobre $Y_{t, x}=Y_{t}$. Defínase $\phi: C_{p}(X, Y) \rightarrow C$ mediante $\phi(f)(t)=p_{t} \circ f$ para todo $t \in T$; se afirma que $\phi$ es un homeomorfismo. El mapeo $\left(p_{t}\right)_{*}: C_{p}(X, Y) \rightarrow C_{p}\left(X, Y_{t}\right)$ dado por $\left(p_{t}\right)_{*}(f)=p_{t} \circ f$ para todos $t \in T$ y $f \in C_{p}(X, Y)$ es continuo por el lema 2.1.9. Puesto que $q_{t} \circ \phi=\left(p_{t}\right)_{*}$ para todo $t \in T$, también $\phi$ es continuo. Dados distintos $f, g \in C_{p}(X, Y)$, existe $x \in X$ tal que $f(x) \neq g(x)$; luego, existe $t \in T$ para el cual $f(x)(t) \neq g(x)(t)$. Por tanto, $\phi(f)(t)(x)=p_{t}(f(x))=f(x)(t) \neq g(x)(t)=\phi(g)(t)(x)$, así que $\phi(f) \neq \phi(g)$.

Para probar que $\phi$ es suprayectivo, tómese $g \in C$; puesto que $g(t) \in C_{p}\left(X, Y_{t}\right)$ para cualquier $t \in T$, se puede definir a $f: X \rightarrow Y$ mediante $f(x)(t)=g(t)(x)$ para todo $x \in X$; como $p_{t} \circ f=q_{t}(g)$ es continua para todo $t$, también $f$ es continua. Según la definición de $\phi$ se obtiene que $\phi(f)=g$, así que $\phi$ es una condensación. Como $C_{p}(X, Y) \subset Y^{X}$, para mostrar la continuidad de $\phi^{-1}$, puede considerársele como un mapeo $\phi^{-1}: C \rightarrow Y^{X}$; así, la continuidad de $\phi^{-1}$ equivale a la de $r_{x} \circ \phi^{-1}$ para todo $x \in X$, y ésto a su vez equivale a la continuidad de $p_{t} \circ r_{x} \circ \phi^{-1}$ para todo $t \in T$. Dada $g \in C$ si $f=\phi^{-1}(g)$, entonces de la igualdad $f(x)(t)=g(t)(x)$ se deduce que $p_{t} \circ r_{x} \circ \phi^{-1}(g)=g(t)(x)=\pi_{t, x} \circ q_{t}(g)$ para todos $x \in X$ y $t \in T$; de la continuidad de $q_{t}$ y $\pi_{t, x}$ se infiere la de $\phi^{-1}$.

2.1.11. Proposición. Si $T$ es un conjunto y $X_{t}$ es un espacio para todo $t \in T$, entonces el espacio $C_{p}\left(\bigoplus_{t \in T} X_{t}, Y\right)$ es homeomorfo a $\prod\left\{C_{p}\left(X_{t}, Y\right): t \in T\right\}$ para cualquier espacio $Y$.

Demostración. Hagamos $C=\prod_{t \in T} C_{p}\left(X_{t}, Y\right)$ y $X=\bigoplus_{t \in T} X_{t}$; aquí se identifica a $X_{t}$ con el respectivo abierto-cerrado de $X$. Se escribirá $q_{t}: C \rightarrow C_{p}\left(X_{t}, Y\right)$ y $r_{x}: Y^{X} \rightarrow Y_{x}=Y$ para las respectivas proyecciones naturales; además, $s_{x, t}: C_{p}\left(X_{t}, Y\right) \rightarrow Y$ y $\pi_{t}: C_{p}(X, Y) \rightarrow C_{p}\left(X_{t}, Y\right)$ están dadas por $s_{x, t}(h)=h(x)$ y $\pi_{t}(f)=f \mid X_{t}$ para todos $t \in T$ y $x \in X_{t}$. Dados $t \in T$ y 
$U=O\left(x_{1}, \ldots, x_{n} ; U_{1}, \ldots, U_{n}\right)_{\left(X_{t}, Y\right)}$, es claro que $\left(\pi_{t}\right)^{-1}(U)=O\left(x_{1}, \ldots, x_{n} ; U_{1}, \ldots, U_{n}\right)_{(X, Y)}$; por eso $\pi_{t}$ es continua. Defínase $\phi: C_{p}(X, Y) \rightarrow C$ mediante $\phi(f)(t)=\pi_{t}(f)$; se verá que $\phi$ es un homeomorfismo. Como $q_{t} \circ \phi=\pi_{t}$ para todo $t \in T$, se obtiene que $\phi$ es continua. Dadas distintas $f, g \in C_{p}(X, Y)$, elíjase $x \in X$ para el cual $f(x) \neq g(x)$ y sea $t \in T$ tal que $x \in X_{t}$; entonces $\pi_{t}(f)=f\left|X_{t} \neq g\right| X_{t}=\pi_{t}(g)$, de donde $\phi(f) \neq \phi(g)$.

Para probar que $\phi$ es suprayectivo, tómese $g \in C$; dada $x \in X$ hállese la única $t \in T$ tal que $x \in X_{t}$ y hágase $f(x)=g(t)(x)$. Como $q_{t}(h)=h(t)$ para todos $t \in T$ y $h \in C$, tenemos que $q_{t}(g)=f \mid X_{t}$, así que $f$ es continua en $X_{t}$ para todo $t \in T$, de donde $f$ es continua; dado que $f \mid X_{t}=\pi_{t}(f)=q_{t}(\phi(f))$ para cualquier $t \in T$ se infiere que $g=\phi(f)$. Para probar que $\phi^{-1}$ es continua, puede considerársele como un mapeo $\phi^{-1}: C \rightarrow Y^{X}$; así la continuidad de $\phi^{-1}$ equivale a la de $r_{x} \circ \phi^{-1}$ para todo $x \in X$. Fijémonos en cualquier punto $x \in X$; existe $t \in T$ tal que $x \in X_{t}$. Como $\phi$ es una biyección, para todo $g \in C$ la única $f \in C_{p}(X, Y)$ para la cual $\phi(f)=g$ cumple $g(t)(x)=f(x)=r_{x}(f)=r_{x}\left(\phi^{-1}(g)\right)$; puesto que $g(t)(x)=q_{t}(g)(x)=s_{x, t} \circ q_{t}(g)$ se deduce que $r_{x} \circ \phi^{-1}=s_{x, t} \circ q_{t}$ para todo $x \in X$. Claramente $s_{x, t}$ y $q_{t}$ son continuas, por lo cual $\phi^{-1}$ también lo es.

Ahora veremos la relación entre $\mathcal{C}$ y la toma de subespacios.

2.1.12. Definición. Sea $Y$ un subespacio de un espacio $X$; el mapeo $\pi_{Y}: C_{p}(X) \rightarrow C_{p}(Y)$ dado por $\pi_{Y}(f)=f \mid Y$ para todo $f \in C_{p}(X)$ se llama mapeo de restricción.

2.1.13. Proposición. Si $X$ es un espacio y $Y \subset X$, entonces:

(i) el mapeo $\pi_{Y}$ es continuo y $\overline{\pi_{Y}\left(C_{p}(X)\right)}=C_{p}(Y)$;

(ii) el mapeo $\pi_{Y}$ es una inyección si y sólo si $\bar{Y}=X$;

(iii) el mapeo $\pi_{Y}$ es un homeomorfismo si y sólo si $Y=X$;

(iv) el mapeo $\pi_{Y}: C_{p}(X) \rightarrow \pi_{Y}\left(C_{p}(X)\right)$ es abierto si y sólo si $Y=\bar{Y}$;

(v) si $X$ es normal y $Y=\bar{Y}$, entonces $\pi_{Y}\left(C_{p}(X)\right)=C_{p}(Y)$.

Demostración. (i) El mapeo $\pi_{Y}$ está bien definido pues es claro que $\pi_{Y}(f) \in C_{p}(Y)$ para todo $f \in C_{p}(X)$. Si $p_{Y}: \mathbb{R}^{X} \rightarrow \mathbb{R}^{Y}$ es la proyección sobre la cara correspondiente y $p_{y}: \mathbb{R}^{Y} \rightarrow \mathbb{R}_{y}=\mathbb{R}$ es la proyección natural para todo $y \in Y$, entonces $p_{y} \circ p_{Y}$ es continua para todo $y$, así que $p_{Y}$ también es continua; puesto que $\pi_{Y}=p_{Y} \mid C_{p}(X)$, resulta que $\pi_{Y}$ es continuo. Por la proposición 2.1.3 y por ser $p_{Y}$ continua, tenemos que $\mathbb{R}^{Y}=p_{Y}\left(\mathbb{R}^{X}\right)=p_{Y}\left(\overline{C_{p}(X)}\right) \subset \overline{p_{Y}\left(C_{p}(X)\right)}$, donde las cerraduras se toman en los espacios correspondientes; por tanto, $\pi_{Y}\left(C_{p}(X)\right)=p_{Y}\left(C_{p}(X)\right)$ es denso en $\mathbb{R}^{Y}$ y por ello en $C_{p}(Y)$.

(ii) Si $\bar{Y} \neq X$, hagamos $u(x)=0$ para todo $x \in X$; tómese $x \in X \backslash \bar{Y}$. Por ser $X$ un espacio de Tychonoff, existe $f \in C_{p}(X)$ tal que $f(x)=1$ y $f(\bar{Y}) \subset\{0\}$; por lo tanto, aunque $u \neq f$ se obtiene $\pi_{Y}(u)=\pi_{Y}(f)$, así que $\pi_{Y}$ no es inyectiva. Ahora, si $\bar{Y}=X$, sean $f, g \in C_{p}(X)$ funciones distintas; entonces $h=f-g \in C_{p}(X)$, de donde $U=h^{-1}(\mathbb{R} \backslash\{0\}) \in \tau(X)$ y $U \neq \emptyset$. Como $\bar{Y}=X$, existe $y \in Y \cap U$; entonces $\pi_{Y}(f)(y)=f(y) \neq g(y)=\pi_{Y}(g)(y)$, lo cual implica que $\pi_{Y}$ es inyectiva.

(iii) Si $Y=X$, entonces $\pi_{Y}$ es el mapeo identidad en $X$. Si $\pi_{Y}$ es un homeomorfismo, por (ii) se tiene que $\bar{Y}=X$; supongamos que $Y \neq X$ y tomemos $x \in X \backslash Y$. Sean $F=\left\{f \in C_{p}(X): f(x)=1\right\}$ y $g \in C_{p}(Y)$; considérese $U=O\left(g ; y_{1}, \ldots, y_{n} ; \epsilon\right)$ arbitrario. Puede hallarse $f \in C_{p}(X)$ tal que 
$f(x)=1$ y $f\left(y_{i}\right)=g\left(y_{i}\right)$ para todo $i \leq n$; por eso, $\pi_{Y}(f) \in U \cap \pi_{Y}(F)$, de donde $\overline{\pi_{Y}(F)}=C_{p}(Y)$. Puesto que claramente $F$ no es denso en $C_{p}(X)$, se deduce que $\left(\pi_{Y}\right)^{-1}$ no es continua, lo cual es una contradicción; por consiguiente, $Y=X$.

(iv) Se escribirá $Z=\pi_{Y}\left(C_{p}(X)\right)$. Supongamos que el conjunto $Y$ es cerrado; tómese $V=$ $O\left(x_{1}, \ldots, x_{n} ; U_{1}, \ldots, U_{n}\right)_{(X, \mathbb{R})}$ arbitrario. Para probar que $\pi_{Y}: C_{p}(X) \rightarrow Z$ es abierto es suficiente verificar que $\pi_{Y}(V) \in \tau(Z)$, por la proposición 2.1.2; sin pérdida de generalidad podemos suponer que existe $k \leq n+1$ para el cual $x_{i} \in Y$ si y sólo si $i<k$; si $W=O\left(x_{1}, \ldots, x_{k-1} ; U_{1}, \ldots, U_{k-1}\right)_{(Y, \mathbb{R})}$, bastará mostrar que $\pi_{Y}(V)=W$. Como evidentemente $\pi_{Y}(V) \subset W$, considérense $f \in W$ y $r_{i} \in U_{i}$ para $i=k, \ldots, n$; por ser $X$ de Tychonoff, puede encontrarse $g \in C_{p}(X)$ que verifica $g \mid Y=f$ y $g\left(x_{i}\right)=r_{i}$ para $i=k, \ldots, n$. Así, $\pi_{Y}(g)=f$ y $g \in V$, lo que implica que $W \subset \pi_{Y}(V)$; por tanto, $\pi_{Y}(V)=W$.

Ahora, supongamos que $\pi_{Y}$ es un mapeo abierto y existe $x \in \bar{Y} \backslash Y$; definamos los conjuntos $U=O(x ;(0,2))_{(X, \mathbb{R})}, V=O(x ;(2,3))_{(X, \mathbb{R})}$ y sus imágenes $G=\pi_{Y}(U)$ y $H=\pi_{Y}(V)$ bajo $\pi_{Y}$. Supongamos también que existe $f \in G \cap H$; entonces existen $g \in U$ y $h \in V$ para las cuales $f=\pi_{Y}(g)=\pi_{Y}(h)$. La función $w=g-h$ está en $C_{p}(X)$ y $w(x) \neq 0$; luego se cumple la igualdad $A=w^{-1}(\mathbb{R} \backslash\{0\}) \in \tau(x, X)$. Como $x \in \bar{Y}$, existe $y \in Y \cap A$; por tanto, $w(y) \neq 0$, lo cual contradice que $g|Y=h| Y$. Esto muestra que $G \cap H=\emptyset$. Veamos que $G$ es denso en $Z$; para ello, sea $P=O\left(y_{1}, \ldots, y_{n} ; W_{1}, \ldots, W_{n}\right)_{(Y, \mathbb{R})} \neq \emptyset$ arbitrario y elíjanse $r_{i} \in W_{i}$ para $i \leq n$. Por ser $X$ de Tychonoff, existe $f \in C_{p}(X)$ tal que $f(x)=1$ y $f\left(y_{i}\right)=r_{i}$ para todo $i$; es claro que $\pi_{Y}(f) \in P \cap G$. Así, $G$ es denso en $Z$ y análogamente se prueba que $H$ es denso en $Z$; pero $G$ y $H$ son abiertos en $Z$, lo cual contradice que $G \cap H=\emptyset$. En conclusión, $Y=\bar{Y}$.

(v) La contención no trivial $C_{p}(Y) \subset \pi_{Y}\left(C_{p}(X)\right)$, es el importante teorema de Tietze-Urysohn sobre extensión de funciones continuas [Engelking, teorema 2.1.8].

A continuación se analiza la relación entre la operación $\mathcal{C}$ y el paso a imágenes continuas.

2.1.14. Definición. Dada una función continua $K: X \rightarrow Y$, defínase $K^{*}: C_{p}(Y) \rightarrow C_{p}(X)$ mediante $K^{*}(f)=f \circ K$ para toda $f \in C_{p}(Y)$; a $K^{*}$ se le conoce como el mapeo dual.

2.1.15. Definición. Se dice que un mapeo sobreyectivo continuo $f: X \rightarrow Y$ es $\mathbb{R}$-cociente si para cualquier $g: Y \rightarrow \mathbb{R}$ la continuidad de $g \circ f$ equivale a la de $g$.

2.1.16. Lema. Cualquier condensación $\mathbb{R}$-cociente $f: X \rightarrow Y$ es un homeomorfismo.

Demostración. Según [Engelking, teorema 2.3.23], puede suponerse que $X$ es un subespacio de $\mathbb{I}^{A}$ para algún $A$; por tanto, a $f^{-1}$ se le puede considerar como el mapeo $f^{-1}: Y \rightarrow \mathbb{I}^{A}$. Sea $\pi_{a}: \mathbb{I}^{A} \rightarrow \mathbb{I}_{a}=\mathbb{I}$ la proyección natural y $g_{a}=\pi_{a} \circ f^{-1}$ para todo $a \in A$; puesto que $g_{a} \circ f=\pi_{a}$ es continua y $f$ es $\mathbb{R}$-cociente, se deduce que $g_{a}$ es continua para todo $a$ y por ello también lo es $f^{-1}$. Por consiguiente, $f$ es un homeomorfismo.

2.1.17. Proposición. Dado un mapeo continuo $K: X \rightarrow Y$ :

(i) el mapeo $K^{*}$ es continuo.

Si además, $K(X)=Y$, entonces,

(ii) el mapeo $K^{*}$ es un homeomorfismo de $C_{p}(Y)$ sobre $K^{*}\left(C_{p}(Y)\right)$; 
(iii) el conjunto $K^{*}\left(C_{p}(Y)\right)$ es cerrado en $C_{p}(X)$ si y sólo si $K$ es un mapeo $\mathbb{R}$-cociente;

(iv) la igualdad $\overline{K^{*}\left(C_{p}(Y)\right)}=C_{p}(X)$ se da, si y sólo si $K$ es una condensación;

(v) la igualdad $K^{*}\left(C_{p}(Y)\right)=C_{p}(X)$ se da, si y sólo si $K$ es un homeomorfismo;

(vi) Si $G: X \rightarrow Z$ es un mapeo continuo sobreyectivo, entonces existe un mapeo continuo $H: Z \rightarrow Y$ tal que $H \circ G=K$ si y sólo si $K^{*}\left(C_{p}(Y)\right) \subset G^{*}\left(C_{p}(Z)\right)$.

Demostración. (i) Tómense $f \in C_{p}(Y)$ y $U=O\left(K^{*}(f) ; x_{1}, \ldots, x_{n} ; \epsilon\right)_{(X)}$ arbitrarios; entonces $V=O\left(f ; K\left(x_{1}\right), \ldots, K\left(x_{n}\right) ; \epsilon\right)_{(Y)} \in \tau\left(f, C_{p}(Y)\right)$ y si $g \in V$, se tiene que $\left|K^{*}(f)\left(x_{i}\right)-K^{*}(g)\left(x_{i}\right)\right|<\epsilon$ para todo $i \leq n$. Por lo tanto, $K^{*}(g) \in U$, de donde $K^{*}(V) \subset U$ y por eso $K^{*}$ es continua.

(ii) Sean $f, g \in C_{p}(Y)$ funciones distintas; entonces existe $y \in Y$ tal que $f(y) \neq g(y)$. Si $x \in K^{-1}(y)$ se obtiene que $K^{*}(f)(x) \neq K^{*}(g)(x)$, así que $K^{*}$ es inyectiva. Para ver que $\left(K^{*}\right)^{-1}$ es continua, tómense $f \in C_{p}(Y)$ y $U=O\left(f ; y_{1}, \ldots, y_{n} ; \epsilon\right)_{(Y)}$ arbitrario; elíjase $x_{i} \in K^{-1}\left(y_{i}\right)$ para todo $i \leq n$ y sea $V=O\left(K^{*}(f) ; x_{1}, \ldots, x_{n} ; \epsilon\right)_{(X)} \cap K^{*}\left(C_{p}(Y)\right)$. Dada $h \in V$ existe $h^{\prime} \in C_{p}(Y)$ para la cual $K^{*}\left(h^{\prime}\right)=h$, de donde $h^{\prime}\left(y_{i}\right)=K^{*}\left(h^{\prime}\right)\left(x_{i}\right)=h\left(x_{i}\right)$ para todo $i \leq n$; puesto que $h \in V$, se deduce que $h^{\prime} \in U$. Por consiguiente, $h \in K^{*}(U)$, lo que implica que $V \subset K^{*}(U)$ y $\left(K^{*}\right)^{-1}(V) \subset U$; o sea que $\left(K^{*}\right)^{-1}$ es continua en $K^{*}(f)$. Por tanto, $K^{*}$ es un encaje.

(iii) Tómense un mapeo $\mathbb{R}$-cociente $K$, y $f \in \overline{K^{*}\left(C_{p}(Y)\right)}$; supongamos que para alguna $y \in Y$ existen $x_{1}, x_{2} \in K^{-1}(y)$ tales que $f\left(x_{1}\right) \neq f\left(x_{2}\right)$. Sea $g \in O\left(f ; x_{1}, x_{2} ; \epsilon\right)_{(X)} \cap K^{*}\left(C_{p}(Y)\right)$, donde $\epsilon=\left|f\left(x_{1}\right)-f\left(x_{2}\right)\right| / 2$; entonces $g\left(x_{1}\right) \neq g\left(x_{2}\right)$ y $g=K^{*}\left(g^{\prime}\right)$ para cierta $g^{\prime} \in C_{p}(Y)$. Por tanto, $g\left(x_{1}\right)=K^{*}\left(g^{\prime}\right)\left(x_{1}\right)=g^{\prime}\left(K\left(x_{1}\right)\right)=g^{\prime}\left(K\left(x_{2}\right)\right)=g\left(x_{2}\right)$, lo cual es una contradicción; luego $f$ es constante en las fibras de $K$. Esto implica que existe $f^{\prime}: Y \rightarrow \mathbb{R}$ que cumple $f=f^{\prime} \circ K$; puesto que $K$ es $\mathbb{R}$-cociente y $f$ es continua, $f^{\prime} \in C_{p}(Y)$, de donde $f=K^{*}\left(f^{\prime}\right) \in K^{*}\left(C_{p}(Y)\right)$, así que $\overline{K^{*}\left(C_{p}(Y)\right)}=K^{*}\left(C_{p}(Y)\right)$.

Ahora, si $\overline{K^{*}\left(C_{p}(Y)\right)}=K^{*}\left(C_{p}(Y)\right)$, sea $f: Y \rightarrow \mathbb{R}$ tal que $g=f \circ K \in C_{p}(X)$. Dados $x_{1}, \ldots, x_{n} \in X$ y $\epsilon>0$, por ser $Y$ espacio de Tychonoff y $g$ constante en las fibras de $K$ puede construirse $h \in C_{p}(Y)$ para la cual $h\left(K\left(x_{i}\right)\right)=g\left(x_{i}\right)=f\left(K\left(x_{i}\right)\right)$ para todo $i \leq n$; por ello, $K^{*}(h) \in O\left(g ; x_{1}, \ldots, x_{n} ; \epsilon\right)_{(X)} \cap K^{*}\left(C_{p}(Y)\right)$, de donde $g \in \overline{K^{*}\left(C_{p}(Y)\right)}=K^{*}\left(C_{p}(Y)\right)$. De modo que $K^{*}(f)=g=K^{*}\left(g^{\prime}\right)$ para alguna $g^{\prime} \in C_{p}(Y)$; aquí $K^{*}: \mathbb{R}^{Y} \rightarrow \mathbb{R}^{X}$ es la extensión de la función original $K^{*}$ a $\mathbb{R}^{Y}$ la cual se calcula de la misma manera. Puesto que la extensión de $K^{*}$ a $\mathbb{R}^{Y}$ también es inyectiva, se deduce que $f=g^{\prime}$; es decir, $f$ es continua, así que $K$ es $\mathbb{R}$-cociente.

(iv) Si $K$ es una condensación, dados $f \in C_{p}(X), x_{1}, \ldots, x_{n} \in X$ y $\epsilon>0$, existe $g \in C_{p}(Y)$ tal que $g\left(K\left(x_{i}\right)\right)=f\left(x_{i}\right)$ para todo $i \leq n$, así que $K^{*}(g) \in O\left(f ; x_{1}, \ldots, x_{n} ; \epsilon\right)_{(X)}$ para todo $\epsilon>0$. Por tanto, $f \in \overline{K^{*}\left(C_{p}(Y)\right)}$, de donde $C_{p}(X)=\overline{K^{*}\left(C_{p}(Y)\right)}$. Ahora, si $K$ no es inyectiva, existen puntos distintos $x_{1}, x_{2}$ para los cuales $K\left(x_{1}\right)=K\left(x_{2}\right)$; puesto que $U=O\left(x_{1}, x_{2} ;(0,1),(2,3)\right)_{(X, \mathbb{R})} \neq \emptyset$ cumple $U \cap K^{*}\left(C_{p}(Y)\right)=\emptyset$, se deduce que $C_{p}(X) \neq \overline{K^{*}\left(C_{p}(Y)\right)}$.

(v) Si $K$ es un homeomorfismo, para cada $f \in C_{p}(X)$ se tiene que $g=f \circ K^{-1} \in C_{p}(Y)$ y que $K^{*}(g)=f$, de donde $C_{p}(X)=K^{*}\left(C_{p}(Y)\right)$. Ahora, si $K^{*}$ es suprayectiva, $K$ es una condensación por (iv), y es $\mathbb{R}$-cociente por (iii); por el lema 2.1.16, se infiere que $K$ es un homeomorfismo.

(vi) Si existe un mapeo continuo $H: Z \rightarrow Y$ tal que $H \circ G=K$, entonces para todo $f \in C_{p}(Y)$ se tiene que $G^{*}\left(H^{*}(f)\right)=f \circ H \circ G=f \circ K=K^{*}(f)$, así que $K^{*}\left(C_{p}(Y)\right) \subset G^{*}\left(C_{p}(Z)\right)$. Ahora, sea $K^{*}\left(C_{p}(Y)\right) \subset G^{*}\left(C_{p}(Z)\right)$; puesto que para cada $f \in C_{p}(Z)$, el mapeo $G^{*}(f)$ es constante en 
las fibras de $G$, también debe serlo $K^{*}(g)$ para cada $g \in C_{p}(Y)$. Supongamos que existen $z \in Z$ y $x, y \in G^{-1}(z)$ para los cuales $K(x) \neq K(y)$; existe $g \in C_{p}(Y)$ que verifica $g(K(x))=0$ y $g(K(y))=1$, lo que implica que $K^{*}(g)$ no es constante en $G^{-1}(z)$, lo cual es una contradicción. Por lo tanto, $K$ es constante en las fibras de $G$ y por ello existe $H: Z \rightarrow Y$ tal que $K=H \circ G$.

Para ver que $H$ es continua, tómese $B \subset Z$ y $z \in \bar{B}$; supongamos que $H(z) \notin \overline{H(B)}$. Así, existe $f \in C_{p}(Y)$ que cumple $f(H(z))=1$ y $f(H(B))=\{0\}$; si $x \in G^{-1}(z)$, se obtiene $K(x)=H(z)$, de donde $K^{*}(f)(x)=1$. Sea $g \in C_{p}(Z)$ tal que $G^{*}(g)=K^{*}(f)$; entonces, $1=K^{*}(f)(x)=g(z)$. Por otro lado, si $b \in B$ y $a \in G^{-1}(b)$ se tiene que $K(a)=H(b) \in H(B)$, así que $K^{*}(f)(a)=0$. En consecuencia, $0=K^{*}(f)(a)=g(b)$ y siendo $b \in B$ arbitraria, $g(B)=\{0\}$; dado que $g(z)=1$, se contradice la continuidad de $g$. Por tanto, $H$ es continua.

Puesto que $C_{p}(X)$ es espacio de Tychonoff para todo $X$, puede también aplicársele la operación $\mathcal{C}$; resulta que $C_{p}\left(C_{p}(X)\right)$ tiene una importante relación con $X$ (teorema 2.1.24).

2.1.18. Definición. Dados $F \subset C_{p}(X)$ y $x \in X$ sea $e_{x}^{F}: F \rightarrow \mathbb{R}$ la función dada por $e_{x}^{F}(f)=f(x)$ para toda $f \in F$; se denota $e_{x}^{C_{p}(X)}$ por $e_{x}$. El mapeo $E^{F}: X \rightarrow \mathbb{R}^{F}$ que se calcula como $E^{F}(x)=e_{x}^{F}$ para todo $x \in X$, se llama mapeo de evaluación.

2.1.19. Definición. Sea $F \subset \mathbb{R}^{X}$; se dice que $F$ separa los puntos en $X$, si para $x, y \in X$ distintos, existe $f \in F$ tal que $f(x) \neq f(y)$. Se dice que $F$ separa los puntos de los conjuntos cerrados en $X$, si dados $x \in X$ y un conjunto cerrado $G \subset X$ tal que $x \notin G$, existe $f \in F$ para la cual $f(x) \notin \overline{f(G)}$. Se dice que $F$ genera $\tau(X)$, si la familia $\left\{f^{-1}(U): f \in F\right.$ y $\left.U \in \tau(\mathbb{R})\right\}$ es una subbase de $\tau(X)$.

2.1.20. Proposición. Si $X$ es un espacio y $F \subset C_{p}(X)$ entonces:

(i) $E^{F}(X) \subset C_{p}(F)$ y $E^{F}$ es un mapeo continuo;

(ii) el mapeo $E^{F}$ es inyectivo si y sólo si $F$ separa los puntos de $X$;

(iii) el mapeo $E^{F}$ es un encaje si y sólo si $F$ genera la topología de $X$;

(iv) el mapeo $E^{F}$ es un encaje si $F$ separa los puntos de los conjuntos cerrados en $X$;

(v) El espacio $X^{\prime}=E^{F}(X) \subset C_{p}(F)$ genera la topología de $F$ (en consecuencia $F$ se encaja en $\left.C_{p}\left(X^{\prime}\right)\right)$.

Demostración. (i) Para cada $x \in X$, la proyección natural $\pi_{x}: \mathbb{R}^{X} \rightarrow \mathbb{R}_{x}=\mathbb{R}$ es continua; por lo tanto, $e_{x}^{F}=\pi_{x} \mid F$ también lo es, lo que significa que $E^{F}(X) \subset C_{p}(F)$. Para probar que $E^{F}$ es continuo, sea $x \in X$ y $U=O\left(e_{x}^{F} ; f_{1}, \ldots, f_{n} ; \epsilon\right)_{(F)}$ una vecindad canónica de $e_{x}^{F}$; entonces $V=\bigcap_{i=1}^{n} f_{i}^{-1}\left(\left(f_{i}(x)-\epsilon, f_{i}(x)+\epsilon\right)\right) \in \tau(x, X)$ y se cumple $E^{F}(V) \subset U$; por ésto, $E^{F}$ es continuo.

(ii) Si $F$ separa los puntos de $X$, dados puntos distintos $x, y \in X$ existe $f \in F$ tal que $e_{x}^{F}(f)=f(x) \neq f(y)=e_{y}^{F}(f)$, de donde $E^{F}(x) \neq E^{F}(y)$. Recíprocamente, si $E^{F}$ es inyectiva, para cualesquiera puntos distintos $x, y \in X$ se tiene que $E^{F}(x) \neq E^{F}(y)$, así que existe $f \in F$ que cumple $f(x)=e_{x}^{F}(f) \neq e_{y}^{F}(f)=f(y)$; por tanto, $F$ separa los puntos en $X$.

(iii) Sea $E^{F}$ un encaje, es decir, un homeomorfismo entre $X$ y $X^{\prime}=E^{F}(X)$; por la proposición 2.1 .2 se tiene que $\mathcal{B}^{\prime}=\{O(f ; U): f \in F$ y $U \in \tau(\mathbb{R})\}$ es una subbase de $C_{p}(F)$. Por lo tanto, $\mathcal{S}^{\prime}=\left\{V \cap X^{\prime}: V \in \mathcal{B}^{\prime}\right\}$ es una subbase de $X^{\prime}$, así que $\mathcal{S}=\left\{\left(E^{F}\right)^{-1}\left(O(f ; U) \cap X^{\prime}\right): f \in F\right.$ y $U \in \tau(\mathbb{R})\}$ es una subbase de $X$. Puesto que $e_{x}^{F} \in O(f ; U)$ si y sólo si $x \in f^{-1}(U)$, se deduce que 
$\left(E^{F}\right)^{-1}\left(O(f ; U) \cap X^{\prime}\right)=f^{-1}(U)$, de donde $\mathcal{S}=\left\{f^{-1}(U): f \in F\right.$ y $\left.U \in \tau(\mathbb{R})\right\}$.

Ahora, si $F$ genera la topología de $X$, dados distintos $x, y \in X$, existen $f_{1}, \ldots, f_{n} \in F$ y $U_{1}, \ldots, U_{n} \in \tau(\mathbb{R})$ tales que $x \in W=\bigcap_{i=1}^{n} f_{i}^{-1}\left(U_{i}\right)$ y $y \notin W$; por tanto, $f_{i}(x) \neq f_{i}(y)$ para alguna $i \leq n$, lo que implica que $F$ separa los puntos de $X$. Por (ii), se tiene que $E^{F}$ es inyectiva. Para probar que $g=\left(E^{F}\right)^{-1}$ es continua en $X^{\prime}=E^{F}(X)$, sean $y \in X^{\prime}, x=g(y)$ y tómese $W \in \tau(x, X)$; según la hipótesis, existen $f_{1}, \ldots, f_{n} \in F$ y $U_{1}, \ldots, U_{n} \in \tau(\mathbb{R})$ para los cuales $x \in G=\bigcap_{i=1}^{n} f_{i}^{-1}\left(U_{i}\right) \subset W$. Para el conjunto $H=\bigcap_{i=1}^{n} O\left(f_{i} ; U_{i}\right) \cap X^{\prime} \in \tau\left(y, X^{\prime}\right)$ fácilmente se prueba que $g(H)=G$, y como $G \subset W$, tenemos que $g=\left(E^{F}\right)^{-1}$ es continua en $y$; por (i), se concluye que $E^{F}$ es un homeomorfismo.

(iv) Sean $x \in X, W \in \tau(x, X)$ y $G=X \backslash W$; entonces existe $f \in F$ tal que $f(x) \notin \overline{f(G)}$, así que $U=\mathbb{R} \backslash \overline{f(G)} \in \tau(f(x), X)$. Por lo tanto, $x \in f^{-1}(U) \subset W$; o sea que $\left\{f^{-1}(U): f \in F\right.$ y $U \in \tau(\mathbb{R})\}$ es base de $X$. De (iii) se sigue que $E^{F}$ es un encaje.

(v) De la proposición 2.1.2, se desprende que la familia $\mathcal{A}=\{O(x, U) \cap F: x \in X$ y $U \in \tau(\mathbb{R})\}$ es una subbase de $F$; puesto que $O(x, U) \cap F=\left(e_{x}^{F}\right)^{-1}(U)$ para cualesquiera $x \in X$ y $U \in \tau(\mathbb{R})$, se tiene que $\mathcal{A}=\left\{\left(e_{x}^{F}\right)^{-1}(U): x \in X\right.$ y $\left.U \in \tau(\mathbb{R})\right\}$. Es claro que $\mathcal{A}=\left\{\phi^{-1}(U): \phi \in X^{\prime}\right.$ y $\left.U \in \tau(\mathbb{R})\right\}$, así que $X^{\prime}$ genera la topología de $F$ por definición; por (iii), se deduce que $F$ se encaja en $C_{p}\left(X^{\prime}\right)$.

A continuación, haremos un estudio más profundo de la operación $\mathcal{C}$, recurriendo para esto a la estructura de anillo topológico de $C_{p}(X)$.

2.1.21. Definición. Una función $\phi: C_{p}(X) \rightarrow \mathbb{R}$ que cumple $\phi(\alpha f+\beta g)=\alpha \phi(f)+\beta \phi(g)$ para cualesquiera $\alpha, \beta \in \mathbb{R}$ y $f, g \in C_{p}(X)$ se llama funcional lineal; la funcional lineal $\phi$ se llama multiplicativa si $\phi(f g)=\phi(f) \phi(g)$ para todos $f, g \in C_{p}(X)$. El conjunto de todas las funcionales lineales con dominio $C_{p}(X)$ y continuas ahí mismo, se denota por $L_{p}(X)$.

2.1.22. Proposición. Dados $n \in \mathbb{N}, x_{i} \in X$ y $\alpha_{i} \in \mathbb{R}$ para $i=1, \ldots, n$, la función $\sum_{i=1}^{n} \alpha_{i} e_{x_{i}}$ es una funcional lineal continua en $C_{p}(X)$.

Demostración. Dados $x \in X, \alpha, \beta \in \mathbb{R}$ y $f, g \in C_{p}(X)$, se cumple $e_{x}(\alpha f+\beta g)=(\alpha f+\beta g)(x)=$ $\alpha f(x)+\beta g(x)=\alpha e_{x}(f)+\beta e_{x}(g)$, así que $e_{x}$ es una funcional lineal para todo $x \in X$; por la proposición 2.1.20(i), cada $e_{x}$ es continua. Es fácil probar que si $\phi_{i} \in L_{p}(X)$ para $i=1, \ldots, n$, entonces $\sum_{i=1}^{n} \phi_{i} \in L_{p}(X)$, y que si $\alpha \in \mathbb{R}$ y $\phi \in L_{p}(X)$, se cumple $\alpha \phi \in L_{p}(X)$, con lo cual se prueba el enunciado.

2.1.23. Lema. El conjunto $L_{p}(X)$ es cerrado en $C_{p}\left(C_{p}(X)\right)$.

Demostración. Tómense $\phi \in \overline{L_{p}(X)}, f, g \in C_{p}(X)$ y $\alpha, \beta \in \mathbb{R}$ arbitrarios. Sean $A=\phi(\alpha f+\beta g)$, $B=\alpha \phi(f)$ y $C=\beta \phi(g)$; se desea probar que $A=B+C$. Dado $\epsilon>0$, para $\epsilon^{\prime}=\epsilon /(|\alpha|+|\beta|+1)$ existe $\phi^{\prime} \in L_{p}(X)$ que satisface las siguientes desigualdades $\left|\phi(f)-\phi^{\prime}(f)\right|<\epsilon^{\prime},\left|\phi(g)-\phi^{\prime}(g)\right|<\epsilon^{\prime}$ y $\left|\phi(\alpha f+\beta g)-\phi^{\prime}(\alpha f+\beta g)\right|<\epsilon^{\prime}$; por tanto, si $A^{\prime}=\phi^{\prime}(\alpha f+\beta g), B^{\prime}=\alpha\left(\phi^{\prime}(f)\right)$ y $C^{\prime}=\beta \phi^{\prime}(g)$, entonces $A^{\prime}=B^{\prime}+C^{\prime} \mathrm{y}|A-B-C| \leq\left|A-A^{\prime}\right|+\left|B-B^{\prime}\right|+\left|C-C^{\prime}\right|+\left|A^{\prime}-B^{\prime}-C^{\prime}\right|<\epsilon^{\prime}+|\alpha| \epsilon^{\prime}+|\beta| \epsilon^{\prime}=\epsilon$. Por ser $\epsilon>0$ arbitrario, se obtiene $A-B-C=0$.

2.1.24. Teorema. Para todo espacio $X$, se puede encajar a $X$ en $C_{p}\left(C_{p}(X)\right)$ como un subespacio cerrado, empleando el mapeo de evaluación $E^{C_{p}(X)}$. 
Demostración. Por ser $X$ espacio de Tychonoff, dados $x \in X$ y un conjunto cerrado $G \subset X \backslash\{x\}$ existe $f \in C_{p}(X)$ tal que $f(x)=1$ y $f(G) \subset\{0\}$, de donde $f(x) \notin \overline{f(G)}$; por la proposición 2.1.20(iv) (haciendo $F=C_{p}(X)$ ), se obtiene que $E=E^{F}: X \rightarrow C_{p}\left(C_{p}(X)\right)$ es un encaje. Se puede suponer que $|X| \geq \omega$, pues en caso contrario el argumento anterior termina la demostración.

Ahora, sea $u \in C_{p}(X)$ la función constante con valor 0 ; supongamos que existe $\phi \in C_{p}\left(C_{p}(X)\right)$ tal que $\phi \in \overline{E(X)} \backslash E(X)$. Por la proposición 2.1.22, tenemos que $E(X) \subset L_{p}(X)$; por el lema 2.1.23, se cumple $\phi \in L_{p}(X)$, así que $\phi(u)=0$. Puesto que $\phi$ es continua, existe $W=O\left(u ; x_{1}, \ldots, x_{n} ; \epsilon\right)$ para el cual $\phi(W) \subset(-1 / 2,1 / 2)$ y se puede suponer que $x_{i} \neq x_{j}$ para $i \neq j$. Dado que $C_{p}\left(C_{p}(X)\right)$ es un espacio regular, pueden elegirse los conjuntos $U_{i} \in \tau\left(x_{i}, X\right)$ ajenos y tales que $\phi \notin \overline{\bigcup\left\{E\left(U_{i}\right): i \leq n\right\}}$. Por ser $X$ un espacio de Tychonoff, para cada $i \leq n$ existe $f_{i} \in C_{p}(X,[0,1])$ tal que $f_{i}\left(x_{i}\right)=1 \mathrm{y}$ $f_{i}\left(X \backslash U_{i}\right)=\{0\}$. Por lo tanto, la función $f=1-\left(f_{1}+\ldots+f_{n}\right) \in C_{p}(X)$ satisface las igualdades $f\left(x_{i}\right)=0$ para todo $i \leq n$ y $f(X \backslash U)=\{1\}$, donde $U=\bigcup_{i=1}^{n} U_{i}$; dado que $f \in W$, se obtiene $\phi(f) \in(-1 / 2,1 / 2)$. Pero por otro lado, $e_{x}(f)=1$ para todo $x \in X \backslash U$, lo que implica que $V=O(f ;(-1 / 2,1 / 2)) \in \tau\left(\phi, C_{p}\left(C_{p}(X)\right)\right)$ cumple $V \cap E(X \backslash U)=\emptyset$; luego, $\phi \notin \overline{E(X \backslash U)}$, por lo cual $\phi \notin \overline{E(X \backslash U)} \cup \overline{E(U)}=\overline{E(X)}$, mismo que es una contradicción. En consecuencia, $E(X)=\overline{E(X)}$.

2.1.25. Definición. Una familia $\mathcal{N}$ de subconjuntos de un espacio $X$ es una red de $X$, si dados $x \in X$ y $U \in \tau(x, X)$, existe $S \in \mathcal{N}$ tal que $x \in S \subset U$; el peso de red de un espacio $X$ es el cardinal $n w(X)=\min \{|\mathcal{N}|: \mathcal{N}$ es una red de $X\}$.

2.1.26. Teorema. Para todo espacio infinito $X$, se tiene que $n w(X)=n w\left(C_{p}(X)\right)$.

Demostración. Sean $\mathcal{N}$ una red en $X$ y $\mathcal{B}$ una base numerable de $\mathbb{R}$; para cada par de familias $\left\{S_{1}, \ldots, S_{k}\right\} \subset \mathcal{N}$ y $\left\{U_{1}, \ldots, U_{k}\right\} \subset \mathcal{B}$ se define $O\left(S_{1}, \ldots, S_{k} ; U_{1}, \ldots, U_{k}\right)=\left\{f \in C(X): f\left(S_{i}\right) \subset U_{i}\right.$ para todo $i \leq k\}$. Sea $\gamma=\left\{O\left(S_{1}, \ldots, S_{k} ; U_{1}, \ldots, U_{k}\right): k \in \mathbb{N}, S_{1}, \ldots, S_{k} \in \mathcal{N}\right.$ y $\left.U_{1}, \ldots, U_{k} \in \mathcal{B}\right\}$ y tómense cualesquiera $f \in C_{p}(X)$ y $O\left(f ; x_{1}, \ldots, x_{k} ; \epsilon\right)$; podemos suponer que los puntos $x_{i}$ son distintos. Para cada $i \leq k$ elíjase $U_{i} \in \mathcal{B}$ tal que $f\left(x_{i}\right) \in U_{i} \subset\left(f\left(x_{i}\right)-\epsilon, f\left(x_{i}\right)+\epsilon\right)$; por la continuidad de $f$, existe $S_{i} \in \mathcal{N}$ para el cual $x_{i} \in S_{i}$ y $f\left(S_{i}\right) \subset U_{i}$, así que $f \in O\left(S_{1}, \ldots, S_{k} ; U_{1}, \ldots, U_{k}\right)$. Tómese $g \in O\left(S_{1}, \ldots, S_{k} ; U_{1}, \ldots, U_{k}\right)$; dado que $x_{i} \in S_{i}$, se obtiene $g\left(x_{i}\right) \in U_{i}$, de donde $\left|g\left(x_{i}\right)-f\left(x_{i}\right)\right|<\epsilon$ para todo $i \leq k$. Por eso, $g \in O\left(f ; x_{1}, \ldots, x_{k} ; \epsilon\right)$, lo que implica que $O\left(S_{1}, \ldots, S_{k} ; U_{1}, \ldots, U_{k}\right) \subset$ $O\left(f ; x_{1}, \ldots, x_{k} ; \epsilon\right)$; es decir, $\gamma$ es una red en $C_{p}(X)$. Es claro que $|\gamma| \leq|\mathcal{N}|$; en consecuencia, $n w\left(C_{p}(X)\right) \leq n w(X)$.

Como en cualquier espacio $Y$, de las contenciones $A \subset B \subset Y$ se sigue que $n w(A) \leq n w(B)$, por el teorema 2.1.24, se deduce que $n w(X) \leq n w\left(C_{p}\left(C_{p}(X)\right)\right)$. Por la última desigualdad del párrafo anterior, $n w\left(C_{p}\left(C_{p}(X)\right)\right) \leq n w\left(C_{p}(X)\right)$, por lo cual $n w(X) \leq n w\left(C_{p}(X)\right)$.

2.1.27. Proposición. Cada funcional lineal continua $\phi$ en $C_{p}(X)$ se puede expresar en la forma $\phi=\sum_{i=1}^{n} \alpha_{i} e_{x_{i}}$ para ciertos $\alpha_{i} \in \mathbb{R} y$ distintos $x_{i} \in X$.

Demostración. Sea $u \in C_{p}(X)$ la función constante con valor 0 ; puesto que $\phi$ es lineal y continua, $\phi(u)=0$ y existen distintos $x_{1}, \ldots, x_{n} \in X$ y $\epsilon>0$ tales que $\phi\left(O\left(u ; x_{1}, \ldots, x_{n} ; \epsilon\right)\right) \subset(-1,1)$. Supongamos que la función $f \in C_{p}(X)$ cumple $f\left(x_{i}\right)=0$ para todo $i \leq n$; dado $k \in \mathbb{N}$, se tiene que $k f \in O\left(u ; x_{1}, \ldots, x_{n} ; \epsilon\right)$, de donde $|\phi(k f)|<1$ y por tanto $|\phi(f)|<1 / k$. Puesto que $k \in \mathbb{N}$ 
es arbitraria, $\phi(f)=0$; por ésto y por ser $\phi$ lineal, se tiene que $\phi(f)=\phi(g)$, para cualesquiera $f, g \in C_{p}(X)$ que verifiquen las igualdades $f\left(x_{i}\right)=g\left(x_{i}\right)$ para todo $i \leq n$.

Para cada $i \leq n$, elíjase $U_{i} \in \tau\left(x_{i}, X\right)$, de tal modo que sean disjuntos; existen funciones $f_{i} \in$ $C(X,[0,1])$ tales que $f_{i}\left(x_{i}\right)=1$ y $f_{i}\left(X \backslash U_{i}\right) \subset\{0\}$ para todo $i \leq n$. Defínase $\alpha_{i}=\phi\left(f_{i}\right)$; tómese $f \in C_{p}(X)$ y sea $g=\sum_{i=1}^{n} f\left(x_{i}\right) f_{i}$. Puesto que $f\left(x_{i}\right)=g\left(x_{i}\right)$ para todo $i \leq n$, se infiere que $\phi(f)=\phi(g)=\sum_{i=1}^{n} \alpha_{i} f\left(x_{i}\right)=\left(\sum_{i=1}^{n} \alpha_{i} e_{x_{i}}\right)(f)$; por lo tanto, $\phi=\sum_{i=1}^{n} \alpha_{i} e_{x_{i}}$.

2.1.28. Proposición. Para cada funcional lineal multiplicativa continua $\phi \in C_{p}\left(C_{p}(X)\right)$ que no sea nula, existe $x \in X$ para el cual $\phi=e_{x}$.

Demostración. Puesto que $\phi \in L_{p}(X)$, por la proposición 2.1.27, existen distintos $x_{1}, \ldots, x_{n} \in X$ y $\alpha_{1}, \ldots, \alpha_{n} \in \mathbb{R}$ que se pueden suponer distintos de 0 , tales que $\phi=\sum_{i=1}^{n} \alpha_{i} e_{x_{i}}$. Supongamos que $n \geq 2$; entonces existen $f, g \in C_{p}(X)$ para las cuales $f\left(x_{1}\right)=1, f\left(x_{k}\right)=0$ para $k \neq 1$ y $g\left(x_{2}\right)=1$, $g\left(x_{k}\right)=0$ para todo $k \neq 2$. Por lo tanto, $\phi(f g)=0$, en tanto que $\phi(f) \phi(g)=\alpha_{1} \alpha_{2} \neq 0$, lo que contradice que $\phi$ es multiplicativa; luego $\phi=\alpha e_{x}$ para cierto $\alpha \in \mathbb{R}$ distinto de 0 y $x \in X$. Sea $v \in C_{p}(X)$ la función constante con valor 1 ; entonces $\alpha^{2}=\phi(v) \phi(v)=\phi(v v)=\phi(v)=\alpha$, de donde $\alpha=1$.

2.1.29. Definición. Dos anillos topológicos $A$ y $B$ son topológicamente isomorfos si existe un homeomorfismo $F: A \rightarrow B$ que a la vez es un isomorfismo algebraico entre $A$ y $B$.

Con esto llegamos al siguiente teorema fundamental:

2.1.30. Teorema (Nagata). Dados espacios $X, Y$, los anillos $C_{p}(X)$ y $C_{p}(Y)$ son topológicamente isomorfos si y sólo si $X$ y $Y$ son homeomorfos.

Demostración. Si $\phi: X \rightarrow Y$ es un homeomorfismo, por la proposición 2.1.17(ii) y 2.1.17(v), el mapeo dual $\phi^{*}: C_{p}(Y) \rightarrow C_{p}(X)$ es un homeomorfismo. Para ver que $\phi$ es un isomorfismo algebraico, sean $f, g \in C_{p}(X)$; entonces $\phi^{*}(f+g)(x)=(f+g)(\phi(x))=f(\phi(x))+g(\phi(x))=$ $\left(\phi^{*}(f)+\phi^{*}(g)\right)(x)$, para todo $x \in X$, así que $\phi^{*}(f+g)=\phi^{*}(f)+\phi^{*}(g)$. Análogamente, se obtiene que $\phi^{*}(f g)=\phi^{*}(f) \phi^{*}(g)$; por tanto, $\phi^{*}$ es un isomorfismo topológico.

Ahora, si $i: C_{p}(X) \rightarrow C_{p}(Y)$ es un isomorfismo topológico, por la proposición 2.1.17 el mapeo dual $i^{*}: C_{p}\left(C_{p}(Y)\right) \rightarrow C_{p}\left(C_{p}(X)\right)$ es un homeomorfismo; por el teorema 2.1.24, es suficiente probar que $i^{*}(E(Y))=E(X)$ donde $E(X)=E^{C_{p}(X)}(X)$ y $E(Y)=E^{C_{p}(Y)}(Y)$. Dada $\xi \in E(Y)$, por la proposición 2.1.22, se obtiene $\xi \in L_{p}(Y)$ y es claro que $\xi$ es multiplicativa; puesto que $i$ es un isomorfismo topológico, el mapeo $i^{*}(\xi)=\xi \circ i$ es una funcional lineal multiplicativa continua en $C_{p}(X)$. Como la función constante de valor 1 está en $C_{p}(Y)$ se tiene que $\xi\left(C_{p}(Y)\right) \neq\{0\}$, lo que implica $i^{*}(\xi)\left(C_{p}(X)\right) \neq\{0\}$; por la proposición 2.1.28, se tiene que $i^{*}(\xi) \in E(X)$, de donde $i^{*}(E(Y)) \subset E(X)$. Análogamente, si $\psi \in E(X)$, entonces $\phi=\psi \circ i^{-1}$ es una funcional lineal multiplicativa continua no nula en $C_{p}(Y)$ y por ello $\phi \in E(Y)$; luego, $\psi=i^{*}(\phi)$, por lo cual $E(X) \subset i^{*}(E(Y))$.

El teorema anterior nos dice que toda la topología de los espacios de Tychonoff se puede estudiar en los espacios $C_{p}(X)$, en el sentido de que para cada propiedad topológica $\mathcal{P}$ de $X$ existe una correspondiente propiedad $\mathcal{Q}$ de anillos topológicos en $C_{p}(X)$ tal que $X$ tiene $\mathcal{P}$ si y sólo si $C_{p}(X)$ 


\section{Capítulo 2. Espacios de funciones}

tiene $\mathcal{Q}$. Esto brinda nuevos métodos de estudio de espacios de Tychonoff ya que a veces puede ser más sencillo determinar si $C_{p}(X)$ tiene la propiedad $\mathcal{Q}$ y por lo tanto averiguar si $X$ tiene la propiedad $\mathcal{P}$. 


\subsection{Caracterizaciones de propiedades de convergencia en $C_{p}(X)$}

En todos los enunciados de esta sección se supondrá que las funciones cardinales sólo toman valores infinitos.

Es natural preguntarse si alguna propiedad de $C_{p}(X)$ puede ser completamente caracterizada mediante propiedades de $X$ y viceversa; el teorema 2.1.26 nos indica que el peso de red es una de ellas. En esta sección primero se presenta una serie de teoremas fundamentales de toda la $C_{p^{-}}$ teoría, en los cuales ciertas funciones cardinales de $C_{p}(X)$ se igualan a ciertas funciones cardinales de $X$; con ésto, se logra entre otras cosas caracterizar $\chi\left(C_{p}(X)\right)$ y $\psi\left(C_{p}(X)\right)$ en términos de $X$. Después, mediante el uso de $\omega$-cubiertas (definición 2.2.10) se caracterizan $t\left(C_{p}(X)\right)$ y la propiedad Fréchet-Urysohn de $C_{p}(X)$.

2.2.1. Definición. El peso de un espacio $X$ es el cardinal $w(X)=\min \{|\mathcal{B}|: \mathcal{B}$ es una base de $X\}$.

2.2.2. Teorema. Para todo espacio $X$, tenemos que $|X|=\chi\left(C_{p}(X)\right)=w\left(C_{p}(X)\right)$.

Demostración. Es fácil probar que $\chi(Y) \leq w(Y)$ para todo espacio $Y$, en particular para $C_{p}(X)$; además, si $\mathcal{B}$ es una base numerable de $\mathbb{R}$ y $\mathcal{U}=\left\{O\left(x_{1}, \ldots, x_{n} ; U_{1}, \ldots, U_{n}\right): n \in \mathbb{N}, x_{i} \in X\right.$ y $U_{i} \in \mathcal{B}$ para todo $\left.i \leq n\right\}$, entonces de la proposición 2.1.2 se sigue que $\mathcal{U}$ es una base de $C_{p}(X)$ y como $X$ es infinito, $|\mathcal{U}| \leq|X|$. Por tanto, $w\left(C_{p}(X)\right) \leq|X|$ así que es suficiente mostrar que $|X| \leq$ $\chi\left(C_{p}(X)\right)$. Sea $u \in C_{p}(X)$ la función constante de valor 0 y $\mathcal{A}$ una base local de $C_{p}(X)$ en $u$ tal que $|\mathcal{A}|=\chi\left(u, C_{p}(X)\right)$; por la proposición 2.1.2, para cada $A \in \mathcal{A}$ puede elegirse $O\left(u ; x_{1}, \ldots, x_{n} ; \epsilon\right) \subset A$. Defínase $\mathcal{D}_{u}$ como la familia de todos los conjuntos básicos así elegidos; entonces $\left|\mathcal{D}_{u}\right| \leq|\mathcal{A}|$. Sea $z(U)=\left\{x_{1}, \ldots, x_{n}\right\}$ para todo $U \in \mathcal{D}_{u}$; hágase $Y=\bigcup\left\{z(U): U \in \mathcal{D}_{u}\right\}$. Supongamos que existe $x \in X \backslash Y$; así, existe $V=O\left(u ; x_{1}, \ldots, x_{n} ; \epsilon\right) \in \mathcal{D}_{u}$ tal que $V \subset W=O(u ; x ; 1)$. Puesto que $z(V)=\left\{x_{1}, \ldots, x_{n}\right\} \subset Y \subset X \backslash\{x\}$, existe $f \in C_{p}(X)$ que cumple $f\left(x_{i}\right)=0$ para todo $i \leq n \mathrm{y}$ $f(x)=1$, por lo cual $f \in V \backslash W$, lo que es una contradicción. En consecuencia $X=Y$ de donde $|X|=|Y|$; dado que $|Y| \leq\left|\mathcal{D}_{u}\right|$, se obtiene $|X| \leq \chi\left(u, C_{p}(X)\right)$. Por el comentario a la proposición 2.1.4, se obtiene $|X| \leq \chi\left(C_{p}(X)\right)$.

2.2.3. Corolario. Para todo espacio $X$, la condición $\chi\left(C_{p}(X)\right) \leq \omega$ implica que $C_{p}(X)$ es metrizable.

Demostración. Por el teorema 2.2.2, se tiene que $w\left(C_{p}(X)\right) \leq \omega$; por [Engelking, teorema 4.2.9], se deduce que $C_{p}(X)$ es metrizable.

2.2.4. Definición. El $i$-peso de un espacio $X$ es el cardinal $i w(X)=\min \{w(Y)$ : existe una condensación $f: X \rightarrow Y\} \cdot \omega$.

2.2.5. Definición. La densidad de un espacio $X$ es el cardinal $d(X)=\min \{|Y|: \bar{Y}=X\}$.

2.2.6. Teorema. Para todo espacio infinito $X$, se verifica que $d(X)=\psi\left(C_{p}(X)\right)=i w\left(C_{p}(X)\right)$.

Demostración. Para probar que $\psi(Z) \leq i w(Z)$ para todo espacio $Z$, supongamos que $f: Z \rightarrow Y$ es una condensación tal que $w(Y)=i w(Z)$; por la proposición 1.1.6, tenemos que $\psi(Z) \leq \psi(Y)$. Por la proposición 1.1 .7 obtenemos $\psi(Y) \leq \chi(Y)$; es claro que $\chi(Y) \leq w(Y)$ de donde $\psi(Z) \leq i w(Z)$. Por eso, $\psi\left(C_{p}(X)\right) \leq i w\left(C_{p}(X)\right)$ y es suficiente mostrar que $i w\left(C_{p}(X)\right) \leq d(X) \leq \psi\left(C_{p}(X)\right)$. 
Ahora, tómese $Y$ tal que $\bar{Y}=X$ y $|Y|=d(X)$; por la proposición 2.1.13, el mapeo de restricción $\pi_{Y}: C_{p}(X) \rightarrow Z=\pi_{Y}\left(C_{p}(X)\right) \subset C_{p}(Y)$ es una condensación. Por tanto, $i w\left(C_{p}(X)\right) \leq w(Z) \leq$ $w\left(C_{p}(Y)\right)$; por el teorema 2.2.2, deducimos que $w\left(C_{p}(Y)\right)=|Y|$ por lo cual, $i w\left(C_{p}(X)\right) \leq d(X)$. Para probar que $d(X) \leq \psi\left(C_{p}(X)\right)$, sea $u \in C_{p}(X)$ la función constante de valor 0 y $\gamma \subset \tau\left(C_{p}(X)\right)$ una familia que cumple $\bigcap \gamma=\{u\}$ y $|\gamma|=\psi\left(C_{p}(X)\right)$; por la proposición 2.1.2, puede suponerse que los elementos de $\gamma$ son de la forma $O\left(u ; x_{1}, \ldots, x_{n} ; \epsilon\right)$. Para cada $V=O\left(u ; x_{1}, \ldots, x_{n} ; \epsilon\right) \in \gamma$ hágase $z(V)=\left\{x_{1}, \ldots, x_{n}\right\}$ y defínase $Y=\bigcup\{z(V): V \in \gamma\}$. Supongamos que existe $x \in X \backslash \bar{Y}$; entonces existe $g \in C_{p}(X)$ tal que $g(x)=1$ y $g(Y)=\{0\}$, de donde $g \in \bigcap \gamma$ pero $g \neq u$ lo cual es una contradicción. Consecuentemente, $X=\bar{Y}$ y como es claro que $|Y| \leq|\gamma|$, se obtiene $d(X) \leq \psi\left(C_{p}(X)\right)$

2.2.7. Ejemplo. El pseudocarácter numerable no se preserva bajo mapeos abiertos. Sean $X=\mathbb{I}^{\omega_{1}}$ y $Y$ la compactación de Alexandroff del espacio discreto de cardinalidad $\omega_{1}$; por el teorema de Hewitt-Marczewski-Pondiczery ([Engelking, teorema 2.3.15]), se tiene que $d(X)=\omega$ y es claro que $d(Y)=\omega_{1}$. Si $Y=\omega_{1} \cup\{p\}$ donde $\{p\}$ es el único punto no aislado de $Y$, puesto que los subconjuntos compactos de $Y \backslash\{p\}$ son precisamente los subconjuntos finitos de $\omega_{1}$, se deduce que $w(Y)=\omega_{1}$; por [Engelking, teorema 2.3.23], tenemos que $Y$ se encaja como subespacio cerrado de $X$. Por 2.1.13(i) y 2.1.13(iv), el mapeo de restricción $\pi_{Y}: C_{p}(X) \rightarrow \pi_{Y}\left(C_{p}(X)\right) \subset C_{p}(Y)$ es abierto; puesto que $X$ es compacto, es normal, así que por la proposición 2.1.13(v), se obtiene $\pi_{Y}\left(C_{p}(X)\right)=C_{p}(Y)$. Por el teorema 2.2.6, se deduce que $\psi\left(C_{p}(X)\right)=d(X)$ y $\psi\left(C_{p}(Y)\right)=d(Y)$; por consiguiente, aunque $\pi_{Y}$ es abierto y $\psi\left(C_{p}(X)\right)=\omega$, se verifica que $\psi\left(\pi_{Y}\left(C_{p}(X)\right)\right)>\omega$.

El siguiente teorema no involucra propiedades de convergencia, pero muestra junto con el teorema 2.2.6 una interesante simetría.

2.2.8. Teorema. Para todo espacio infinito $X$, se tiene que $d\left(C_{p}(X)\right)=i w(X)$.

Demostración. El teorema 2.1.24, implica que $i w(X) \leq i w\left(C_{p}\left(C_{p}(X)\right)\right)$; por el teorema 2.2.6, se obtiene $i w\left(C_{p}\left(C_{p}(X)\right)\right)=d\left(C_{p}(X)\right)$, así que $i w(X) \leq d\left(C_{p}(X)\right)$. Ahora, sea $f: X \rightarrow Y$ una condensación tal que $w(Y)=i w(X)$; por la proposición 2.1.17(iv) se verifica la igualdad $C_{p}(X)=\bar{Z}$, donde $Z=f^{*}\left(C_{p}(Y)\right)$. Por tanto, $d\left(C_{p}(X)\right) \leq d(Z)$ y es claro que $d(Z) \leq n w(Z)$; por la proposición 2.1.17(ii), se cumple $n w(Z)=n w\left(C_{p}(Y)\right)$ y por el teorema 2.1.26, se obtiene $n w\left(C_{p}(Y)\right)=n w(Y)$. Puesto que $n w(Y) \leq w(Y)$, en resumen se tiene que $d\left(C_{p}(X)\right) \leq w(Y) \leq i w(X)$

2.2.9. Definición. El número de Lindelöf de un espacio $X$ es el cardinal $l(X)=\min \{\kappa:$ cada cubierta abierta $\gamma$ de $X$ tiene una subcubierta $\mu$ tal que $|\mu| \leq \kappa\}$.

2.2.10. Definición. Una familia $\gamma$ es una $\omega$-cubierta del espacio $X$ si para cada conjunto finito $A \subset X$, existe $U \in \gamma$ para el cual $A \subset U$.

2.2.11. Proposición. Si $X$ es un espacio y $\kappa$ es un cardinal infinito, entonces las siguientes propiedades son equivalentes:

(i) para cada $\omega$-cubierta abierta $\gamma$ de $X$ existe una $\omega$-cubierta $\mu \subset \gamma$ de $X$ tal que $|\mu| \leq \kappa$.

(ii) $l\left(X^{n}\right) \leq \kappa$ para todo $n \in \mathbb{N}$. 
Demostración. (i) $\Rightarrow$ (ii). Sea $\gamma$ una cubierta abierta de $X^{n}$ para algún $n \in \mathbb{N}$. Una familia $\mu \subset \tau(X)$ se llamará $\gamma$-pequeña si dados $U_{1}, \ldots, U_{n} \in \mu$ (no necesariamente distintos), existe $G \in \gamma$ tal que $U_{1} \times \ldots \times U_{n} \subset G$. Sea $\delta=\{\bigcup \mu: \mu$ es una familia $\gamma$-pequeña finita $\}$. Si $A \subset X$ es un conjunto finito, el conjunto $\mathcal{A}=\left\{\left(x_{1}, \ldots, x_{n}\right): x_{i} \in A\right.$ para todo $\left.i \leq n\right\}$ es finito; dada $\left(x_{1}, \ldots, x_{n}\right) \in \mathcal{A}$, para cada $i \leq n$ elíjase $U_{i} \in \tau\left(x_{i}, X\right)$ de tal manera que $U_{1} \times \ldots \times U_{n} \subset G$ para alguna $G \in \gamma$. Sea $\mathcal{V}$ la familia de los productos $U_{1} \times \ldots \times U_{n}$ así obtenidos y para cada $x \in A$, sea $\mathcal{U}_{x}=\{U \in \tau(x, X): U$ es un factor de algún $\left.U_{1} \times \ldots \times U_{n} \in \mathcal{V}\right\}$; entonces $\mathcal{U}_{x}$ es finito y $U_{x}=\bigcap \mathcal{U}_{x} \in \tau(X)$. Por tanto, $\mu=\left\{U_{x}: x \in A\right\}$ es una familia $\gamma$-pequeña finita, y como $A \subset \bigcup \mu$, se deduce que $\delta$ es una $\omega$-cubierta abierta de $X$; por (i), existe una $\omega$-cubierta $\delta^{\prime} \subset \delta$ que cumple $\left|\delta^{\prime}\right| \leq \kappa$. Por definición de $\delta$, para cada $U \in \delta^{\prime}$ existe una familia $\gamma$-pequeña finita $\mu_{U}$ para la cual $U=\bigcup \mu_{U}$; por tanto, existe una familia finita $\gamma_{U} \subset \gamma$ tal que para cualesquiera $W_{1}, \ldots, W_{n} \in \mu_{U}$ se tiene que $W_{1} \times \ldots \times W_{n} \subset G$ para algún $G \in \gamma_{U}$. La familia $\gamma^{\prime}=\bigcup\left\{\gamma_{U}: U \in \delta^{\prime}\right\}$ satisface las condiciones $\gamma^{\prime} \subset \gamma$ y $\left|\gamma^{\prime}\right| \leq \kappa$; se afirma que $X^{n}=\bigcup \gamma^{\prime}$. Si $x=\left(x_{1}, \ldots, x_{n}\right) \in X^{n}$, existe $U \in \delta^{\prime}$ para el cual $\left\{x_{1}, \ldots, x_{n}\right\} \subset U$; luego, para cada $i \leq n$ existe $U_{i} \in \mu_{U}$ que cumple $x_{i} \in U_{i}$. En consecuencia, existe $G \in \gamma_{U}$ que verifica $U_{1} \times \ldots \times U_{n} \subset G$ de donde $x \in G \subset \bigcup \gamma^{\prime}$; por consiguiente $l\left(X^{n}\right) \leq \kappa$.

(ii) $\Rightarrow$ (i). Sea $\gamma$ una $\omega$-cubierta abierta de $X$; entonces para cada $n \in \mathbb{N}$, la familia $\left\{U^{n}: U \in \gamma\right\}$ cubre a $X^{n}$. Puesto que $l\left(X^{n}\right) \leq \kappa$, existe $\gamma_{n}^{\prime} \subset \gamma$ tal que $\left|\gamma_{n}^{\prime}\right| \leq \kappa$ y $\left\{U^{n}: U \in \gamma_{n}^{\prime}\right\}$ cubre a $X^{n}$. Para la familia $\gamma^{\prime}=\bigcup\left\{\gamma_{n}^{\prime}: n \in \mathbb{N}\right\}$ tenemos que $\left|\gamma^{\prime}\right| \leq \kappa$ y $\gamma^{\prime} \subset \gamma$ es una $\omega$-cubierta de $X$, pues dados $n \in \mathbb{N}$ y $A=\left\{x_{1}, \ldots, x_{n}\right\} \subset X$, como $x=\left(x_{1}, \ldots, x_{n}\right) \in X^{n}$, existe $U \in \gamma^{\prime}$ para el cual $x \in U^{n}$. Consecuentemente, $A \subset U$.

2.2.12. Teorema (Arhangel'skit-Pytkeev). Para todo espacio $X$, se satisface la igualdad $t\left(C_{p}(X)\right)=$ $\sup \left\{l\left(X^{n}\right): n \in \mathbb{N}\right\}$.

Demostración. Sean $\lambda=t\left(C_{p}(X)\right)$ y $\kappa=\sup \left\{l\left(X^{n}\right): n \in \mathbb{N}\right\}$; primero se probará que $\lambda \leq \kappa$. Para ello, sea $u$ la función constante de valor 0 y sea $A \subset C_{p}(X)$ tal que $u \in \bar{A}$; según la observación a la proposición 2.1.4, bastará mostrar que existe $B \subset A$ que cumple $|B| \leq \kappa \mathrm{y} u \in \bar{B}$. Para cada $n \in \mathbb{N}$, la familia $\gamma_{n}=\left\{f^{-1}((-1 / n, 1 / n)): f \in A\right\}$ es una $\omega$-cubierta abierta de $X$, porque $u \in \bar{A}$; por la proposición 2.2.11, existe una $\omega$-cubierta $\mu_{n} \subset \gamma_{n}$ tal que $\left|\mu_{n}\right| \leq \kappa$. Elíjase un conjunto $B_{n} \subset A$ para el cual $\mu_{n}=\left\{f^{-1}((-1 / n, 1 / n)): f \in B_{n}\right\}$ y $\left|B_{n}\right| \leq \kappa$. Sea $B=\bigcup\left\{B_{n}: n \in \mathbb{N}\right\}$; es claro que $|B| \leq \kappa$ y se verá que $u \in \bar{B}$. Dados $x_{1}, \ldots, x_{k} \in X$ y $\epsilon>0$, tomemos un número $n \in \mathbb{N}$ que cumpla $1 / n<\epsilon$; por ser $\mu_{n}$ una $\omega$-cubierta de $X$, existe $f \in B_{n}$ tal que $\left\{x_{1}, \ldots, x_{k}\right\} \subset f^{-1}((-1 / n, 1 / n))$, es decir, $\left|f\left(x_{i}\right)\right|<1 / n$ para todo $i \leq n$. Por lo tanto, $f \in O\left(u ; x_{1}, \ldots, x_{k} ; \epsilon\right)$, lo que implica $u \in \bar{B}$.

Ahora se probará que $\kappa \leq \lambda$. Sea $\gamma$ una $\omega$-cubierta abierta de $X$; por la proposición 2.2.11, será suficiente hallar una $\omega$-cubierta $\mu \subset \gamma$ que cumpla $|\mu| \leq \lambda$. Sean $A=\left\{f \in C_{p}(X): f^{-1}(\mathbb{R} \backslash\{0\}) \subset U\right.$ para alguna $U \in \gamma\}$ y $w$ la función constante con valor 1. Para ver que $w \in \bar{A}$ tomemos cualesquiera puntos $x_{1}, \ldots, x_{k} \in X$ y $\epsilon>0$; existe $U \in \gamma$ que cumple $\left\{x_{1}, \ldots, x_{k}\right\} \subset U$. Sea $f \in C_{p}(X)$ tal que $f\left(x_{i}\right)=1$ para todo $i \leq n$ y $f(X \backslash U) \subset\{0\}$; entonces $f \in A \cap O\left(w ; x_{1}, \ldots, x_{k} ; \epsilon\right)$. Puesto que $t\left(C_{p}(X)\right)=\lambda$, existe $B \subset A$ que verifica $|B| \leq \lambda$ y $w \in \bar{B}$; para cada $f \in B$ elíjase $U_{f} \in \gamma$ para el cual $f^{-1}(\mathbb{R} \backslash\{0\}) \subset U_{f}$. El conjunto $\mu=\left\{U_{f}: f \in B\right\}$ cumple $\mu \subset \gamma$ y $|\mu| \leq \lambda$; para probar que $\mu$ es $\omega$-cubierta de $X$, tómese un conjunto finito $F \subset X$. Como $w \in \bar{B}$, existe $f \in B$ tal que $f(x)>0$ para todo $x \in F$; por tanto, $F \subset f^{-1}(\mathbb{R} \backslash\{0\}) \subset U_{f} \in \mu$. 
2.2.13. Corolario. Para todo espacio $X$ tenemos que $t\left(C_{p}(X)\right)=t\left(\left(C_{p}(X)\right)^{\omega}\right)$.

Demostración. Sean $X_{i}=X$ para todo $i \in \omega$ y $Y=\bigoplus_{i \in \omega} X_{i}$; entonces $\left(C_{p}(X)\right)^{\omega}$ y $C_{p}(Y)$ son homeomorfos por la proposición 2.1.11. Sea $t\left(C_{p}(X)\right)=\kappa$; por el teorema 2.2.12, se tiene que $l\left(X^{n}\right) \leq \kappa$ para todo $n \in \mathbb{N}$. Por ser $Y^{n}$ la unión numerable de espacios homeomorfos a $X^{n}$, se deduce que $l\left(Y^{n}\right) \leq \kappa$ para todo $n \in \mathbb{N}$; de nuevo por el teorema 2.2.12, se tiene que $t\left(\left(C_{p}(X)\right)^{\omega}\right)=t\left(C_{p}(Y)\right) \leq \kappa=t\left(C_{p}(X)\right)$. Debido a que $C_{p}(X)$ se encaja en $\left(C_{p}(X)\right)^{\omega}$, se obtiene $t\left(C_{p}(X)\right) \leq t\left(\left(C_{p}(X)\right)^{\omega}\right)$ y en suma $t\left(C_{p}(X)\right)=t\left(\left(C_{p}(X)\right)^{\omega}\right)$.

2.2.14. Ejemplo. Espacio $X$ para el cual $t(X)>\psi(X)$. Sea $v(\alpha)=1$ para todo $\alpha \in \omega_{1}$; hágase $Y=\{0,1\}^{\omega_{1}} \backslash\{v\}$ y $X=C_{p}(Y)$. Para cada $\alpha \in \omega_{1}$, se escribirá $P_{\alpha}=\prod_{\beta \in \omega_{1}} U_{\beta}^{\alpha}$, donde $U_{\alpha}^{\alpha}=\{0\}$ y $U_{\beta}^{\alpha}=\{0,1\}$ para $\beta \neq \alpha$. Por [Engelking, teorema 2.3.15], se tiene que $d\left(\{0,1\} \omega_{1}\right) \leq \omega$, así que $d(Y) \leq \omega$; se sigue del teorema 2.2.6 que $\psi(X) \leq \omega$. Sea $\mathcal{U}=\left\{P_{\alpha} \cap Y: \alpha \in \omega_{1}\right\}$; entonces $\mathcal{U} \subset \tau(Y)$. La familia $\mathcal{U}$ cubre a $Y$, porque si $x=\left(x_{\beta}\right)_{\beta \in \omega_{1}} \in Y$, existe $\alpha \in \omega_{1}$ tal que $x_{\alpha}=0$; por tanto, $x \in P_{\alpha}$. Sin embargo, $\mathcal{U}$ no tiene subcubierta numerable, pues si $\mathcal{V}=\left\{P_{\alpha} \cap Y: \alpha \in A\right\}$, en donde $A \subset \omega_{1}$ es numerable, existe $\alpha \in \omega_{1} \backslash A$; el punto $y=\left(y_{\beta}\right)_{\beta \in \omega_{1}}$ dado por $y_{\alpha}=0$ y $y_{\beta}=1$ para $\beta \neq \alpha$, cumple $y \in Y \backslash \bigcup \mathcal{V}$. En consecuencia, $l(Y)>\omega$; por el teorema 2.2.12, se obtiene $t(X)=t\left(C_{p}(Y)\right)>\omega \geq \psi(X)$.

El teorema 2.2.12 y el siguiente presentan cierta simetría.

2.2.15. Teorema (Asanov). Si $X$ es un espacio, entonces se verifica $t\left(X^{n}\right) \leq l\left(C_{p}(X)\right)$ para todo $n \in \mathbb{N}$.

Demostración. Sean $\kappa=l\left(C_{p}(X)\right)$ y $n \in \mathbb{N}$; para probar la desigualdad $t\left(X^{n}\right) \leq \kappa$ tómense $x=\left(x_{1}, \ldots, x_{n}\right) \in X^{n}$ y $A \subset X^{n}$ tales que $x \in \bar{A}$. Elíjase $O_{i} \in \tau\left(x_{i}, X\right)$ de tal manera que $O_{i} \cap O_{j}=\emptyset$ si $x_{i} \neq x_{j}$ y $O_{i}=O_{j}$ si $x_{i}=x_{j}$; dado que $O=O_{1} \times \ldots \times O_{n} \in \tau(x, X)$ y $x \in \overline{A \cap O}$, se puede suponer que $A \subset O$. Se checa fácilmente que el conjunto $\Phi=\left\{f \in C_{p}(X): f\left(x_{i}\right)=1\right.$ para todo $i \leq n\}$ es cerrado en $C_{p}(X)$, así que $l(\Phi) \leq \kappa$; para cada $y=\left(y_{1}, \ldots, y_{n}\right) \in A$, hágase $U_{y}=\left\{g \in C_{p}(X): g\left(y_{i}\right)>0\right.$ para todo $\left.i \leq n\right\}$. Si $f \in \Phi$, se tiene que $U_{i}=f^{-1}((0, \infty)) \in \tau\left(x_{i}, X\right)$ para todo $i \leq n$; dado que $x \in \bar{A}$, existe $y=\left(y_{1}, \ldots, y_{n}\right) \in A \cap\left(U_{1} \times \ldots \times U_{n}\right)$. Por tanto, $f\left(y_{i}\right)>0$ para todo $i \leq n$, así que $f \in U_{y}$; ésto muestra que $\Phi \subset \bigcup\left\{U_{y}: y \in A\right\}$. La desigualdad $l(\Phi) \leq \kappa$ implica que existe $B \subset A$ tal que $|B| \leq \kappa$ y $\Phi \subset \bigcup\left\{U_{y}: y \in B\right\}$. Para demostrar que $x \in \bar{B}$ tómese $V=V_{1} \times \ldots \times V_{n} \in \tau(x, X)$; puede suponerse que $V_{i} \subset O_{i}$ para todo $i \leq n$ y que $V_{i}=V_{j}$ si $x_{i}=x_{j}$. Por ser $X$ espacio de Tychonoff, existe $g \in \Phi$ para la cual $g\left(X \backslash \bigcup_{i=1}^{n} V_{i}\right) \subset\{0\}$; por lo tanto, $g \in U_{y}$ para alguna $y=\left(y_{1}, \ldots, y_{n}\right) \in B$. Tenemos que $g\left(y_{i}\right)>0$ para todo $i \leq n$, de lo cual se sigue que $y_{i} \in V_{j} \subset O_{j}$ para alguna $j \leq n$; como $y \in A$ implica $y_{i} \in O_{i}$, tenemos que $y_{i} \in O_{i} \cap O_{j}$. Puesto que $O_{i} \cap O_{j}=\emptyset$ cuando $x_{i} \neq x_{j}$, debe ser $x_{i}=x_{j}$; luego $V_{i}=V_{j}$, así que $y_{i} \in V_{i}$ para cada $i \leq n$. En consecuencia, $y \in\left(V_{1} \times \ldots \times V_{n}\right) \cap B$, es decir, $x \in \bar{B}$.

Pero debe tenerse en cuenta que puede suceder que $l\left(C_{p}(X)\right) \neq \sup \left\{t\left(X^{n}\right): n \in \mathbb{N}\right\}$ como se muestra a continuación.

2.2.16. Ejemplo. Sea $X=\omega_{1}$ con la topología discreta; entonces $X^{n}$ también es un espacio discreto para todo $n \in \mathbb{N}$ por lo cual $t\left(X^{n}\right) \leq \omega$. Para cerciorarnos de que $l\left(C_{p}(X)\right)>\omega$ es suficiente probar que $\mathbb{R}^{\omega_{1}}$ no es normal, pues $C_{p}\left(\omega_{1}\right)=\mathbb{R}^{\omega_{1}}$ y todo espacio Lindelöf regular es 
normal; por ser $\mathbb{N}^{\omega_{1}}$ subespacio cerrado de $\mathbb{R}^{\omega_{1}}$ basta probar que $\mathbb{N}^{\omega_{1}}$ no es normal. Para ésto sean $F=\left\{x \in \mathbb{N}^{\omega_{1}}:\left|x^{-1}(i)\right| \leq 1\right.$ para todo $\left.i \neq 1\right\}$ y $G=\left\{x \in \mathbb{N}^{\omega_{1}}:\left|x^{-1}(i)\right| \leq 1\right.$ para todo $i \neq 2\}$; los conjuntos $F$ y $G$ son cerrados. Se probará ésto para $F$, la demostración para $G$ es análoga. Si $x \in \mathbb{N}^{\omega_{1}} \backslash F$ existen distintos $\alpha, \beta \in \omega_{1}$ tales que $x(\alpha)=x(\beta)=i \neq 1$; por tanto, $U=\left\{y \in \mathbb{N}^{\omega_{1}}: y(\alpha)=y(\beta)=i\right\} \in \tau\left(\mathbb{N}^{\omega_{1}}\right)$, mientras $x \in U$ y $U \cap F=\emptyset$.

Si $x \in F$ entonces existen distintos $\alpha, \beta \in \omega_{1}$ para los cuales $x(\alpha)=x(\beta)=1$, así que $x \notin G$, de donde $F \cap G=\emptyset$. Supongamos que $\mathbb{N}^{\omega_{1}}$ es normal; entonces existen $U, V \in \tau\left(\mathbb{N}^{\omega_{1}}\right)$ tales que $F \subset U, G \subset V$ y $U \cap V=\emptyset$. De la proposición 2.1.2 se sigue que la familia de conjuntos abiertos $\mathcal{B}=\left\{O\left(\alpha_{1}, \ldots, \alpha_{n} ;\left\{i_{1}\right\}, \ldots,\left\{i_{n}\right\}\right): \alpha_{k} \in \omega_{1}, i_{k} \in \mathbb{N}, k=1, \ldots, n\right.$ y $\left.n \in \mathbb{N}\right\}$ es una base de $\mathbb{N}^{\omega_{1}}$; sea $\gamma$ una subfamilia disjunta maximal de $\mathcal{B}$ tal que $\bigcup \gamma \subset U$. Es claro que $\overline{\bigcup \gamma}=\bar{U}$; de la proposición 2.1.7 fácilmente se obtiene $c\left(\mathbb{N}^{\omega_{1}}\right) \leq \omega$, de modo que $|\gamma| \leq \omega$. Para cada $W=$ $O\left(\alpha_{1}, \ldots, \alpha_{n} ;\left\{i_{1}\right\}, \ldots,\left\{i_{n}\right\}\right) \in \gamma$ sea $z(W)=\left\{\alpha_{1}, \ldots, \alpha_{n}\right\}$. Considérense el conjunto $A=\bigcup\{z(W)$ : $W \in \gamma\}$ y la proyección $p_{A}: \mathbb{N}^{\omega_{1}} \rightarrow \mathbb{N}^{A}$ sobre la cara correspondiente; entonces $|A| \leq \omega$.

Se afirma que $p_{A}^{-1}\left(p_{A}(\bar{U})\right)=\bar{U}$; para ello basta probar la inclusión $p_{A}^{-1}\left(p_{A}(\bar{U})\right) \subset \bar{U}$. Sea $p_{\alpha}$ : $\mathbb{N}^{\omega_{1}} \rightarrow \mathbb{N}$ la proyección natural para todo $\alpha \in \omega_{1}$; si $x \in p_{A}^{-1}\left(p_{A}(\bigcup \gamma)\right)$, existe $y \in \bigcup \gamma$ tal que $p_{A}(x)=p_{A}(y)$. Supongamos que $y \in W=O\left(\alpha_{1}, \ldots, \alpha_{n} ;\left\{i_{1}\right\}, \ldots,\left\{i_{n}\right\}\right) \in \gamma$; entonces $p_{\alpha_{k}}(x)=$ $p_{\alpha_{k}}(y)$ para $k \leq n$, por lo cual $x \in W \subset \bigcup \gamma$. En consecuencia, $p_{A}^{-1}\left(p_{A}(\bigcup \gamma)\right) \subset \bigcup \gamma$, así que $p_{A}^{-1}\left(p_{A}(\bigcup \gamma)\right)=\bigcup \gamma$. Tómense $x \in \mathbb{N}^{\omega_{1}}$ tal que $y=p_{A}(x) \in \overline{p_{A}(\bigcup \gamma)}$ y $V \in \tau\left(x, \mathbb{N}^{\omega_{1}}\right)$; dado que $p_{A}$ es un mapeo abierto, se tiene que $p_{A}(V) \in \tau\left(y, \mathbb{N}^{A}\right)$. Por lo tanto, $p_{A}(V) \cap p_{A}(\bigcup \gamma) \neq \emptyset$, de donde $V \cap p_{A}^{-1}\left(p_{A}(\bigcup \gamma)\right) \neq \emptyset$; luego $x \in \overline{p_{A}^{-1}\left(p_{A}(\bigcup \gamma)\right)}$, lo cual implica que $p_{A}^{-1}\left(\overline{p_{A}(\bigcup \gamma)}\right) \subset$ $\overline{p_{A}^{-1}\left(p_{A}(\bigcup \gamma)\right)}=\overline{\bigcup \gamma}=\bar{U}$. Por la continuidad de $p_{A}$, se obtiene $p_{A}(\bar{\bigcup} \gamma) \subset \overline{p_{A}(\bigcup \gamma)}$ y por eso $p_{A}^{-1}\left(p_{A}(\bar{U})\right)=p_{A}^{-1}\left(p_{A}(\bar{\bigcup} \gamma)\right) \subset p_{A}^{-1}\left(\overline{p_{A}(\bigcup \gamma)}\right) \subset \bar{U}$.

Dada una numeración $\left\{\alpha_{n}: n \in \mathbb{N}\right\}$ del conjunto $A$ hagamos $x(\beta)=2$ para todo $\beta \in \omega_{1} \backslash A$ y $x\left(\alpha_{n}\right)=n$ para cada $n \in \mathbb{N}$. Además, sea $y(\beta)=1$ para todo $\beta \in \omega_{1} \backslash A$ y $y\left(\alpha_{n}\right)=n$ si $n \in \mathbb{N}$. Para los puntos $x, y \in \mathbb{N}^{\omega_{1}}$ tenemos que $x \in G \subset V$ y $y \in F \subset U$, mientras $p_{A}(x)=p_{A}(y)$; en consecuencia, $y \in \bar{U}$ y $x \in p_{A}^{-1}\left(p_{A}(\bar{U})\right)=\bar{U}$. Por consiguiente, $U \cap V \neq \emptyset$, lo cual es una contradicción; se concluye que $\mathbb{N}^{\omega_{1}}$ no es normal.

2.2.17. Definición. Dada una sucesión $\gamma=\left\{A_{n}: n \in \omega\right\}$ de subconjuntos de $X$, se denotará por $\lim \gamma$ al conjunto $\left\{x \in X\right.$ : existe $m \in \omega$ tal que $x \in A_{n}$ para todo $\left.n \geq m\right\}$.

Se observa que existen otras definiciones de límite de familias de conjuntos, como son el límite directo y el límite inverso de una familia indexada de conjuntos; para aplicaciones del segundo tipo de límite a la Topología ver [Engelking, capítulo 2 sección 5].

2.2.18. Teorema. Para todo espacio $X$, las siguientes condiciones son equivalentes:

(i) el espacio $C_{p}(X)$ es Fréchet-Urysohn;

(ii) para cada $\omega$-cubierta abierta $\gamma$ de $X$, existe una sucesión $\xi=\left\{U_{n}: n \in \omega\right\} \subset \gamma$ tal que $\lim \xi=X$;

(iii) dada una sucesión $\left\{\gamma_{n}: n \in \omega\right\}$ de $\omega$-cubiertas abiertas de $X$, para cada $n \in \omega$ puede elegirse $U_{n} \in \gamma_{n}$ de tal manera que $\lim \left\{U_{n}: n \in \omega\right\}=X$.

Demostración. (i) $\Rightarrow\left(\right.$ ii). Si $X \in \gamma$, tómese $\xi=\left\{U_{n}: n \in \omega\right\}$ donde $U_{n}=X$ para todo $n$; si no, sea 
$z(f)=f^{-1}(\mathbb{R} \backslash\{0\})$ para toda $f \in C_{p}(X)$ y considérese el conjunto $P=\left\{f \in C_{p}(X): z(f) \subset U\right.$ para alguna $U \in \gamma\}$. Se probará que $u \in \bar{P} \backslash P$, siendo $u$ la función constante de valor 1 ; como $z(u)=X \notin \gamma$ se tiene que $u \notin P$. Dados $x_{1}, \ldots, x_{n} \in X$ y $\epsilon>0$, existe $U \in \gamma$ tal que $\left\{x_{1}, \ldots, x_{n}\right\} \subset U$; puesto que puede hallarse $f \in C_{p}(X)$ que cumple $f\left(x_{i}\right)=1$ para todo $i \leq n \mathrm{y}$ $f(X \backslash U)=\{0\}$, se deduce que $f \in O\left(u ; x_{1}, \ldots, x_{n} ; \epsilon\right) \cap P$; por tanto, $u \in \bar{P}$.

Por (i), existe una sucesión $\left\{f_{n}: n \in \omega\right\} \subset P$ que converge a $u$; luego, existe $U_{n} \in \gamma$ que cumple $z\left(f_{n}\right) \subset U_{n}$ para cada $n \in \omega$. Defínase la familia $\xi=\left\{U_{n}: n \in \omega\right\}$; si $x \in X$, tenemos que $W=O(u ; x ; 1 / 2) \in \tau\left(u, C_{p}(X)\right)$, así que existe $N \in \omega$ para el cual $f_{n} \in W$ si $n \geq N$. Ésto implica que $f_{n}(x) \neq 0$ y por ello $x \in z\left(f_{n}\right) \subset U_{n}$ para todo $n \geq N$; en consecuencia, $\lim \xi=X$.

(ii) $\Rightarrow$ (iii). Dadas $\mathcal{U}, \mathcal{U}^{\prime} \subset \tau(X)$, se escribirá $\mathcal{U}<\mathcal{U}^{\prime}$ si para cada $U \in \mathcal{U}$, existe $U^{\prime} \in \mathcal{U}^{\prime}$ tal que $U \subset U^{\prime}$. Se dirá que una sucesión $\left\{\mu_{n}: n \in \omega\right\}$ de $\omega$-cubiertas abiertas de $X$ es especial si para toda $n \in \omega$ existe $U_{n} \in \mu_{n}$ tal que $\lim \left\{U_{n}: n \in \omega\right\}=X$; asimismo, de una sucesión $\left\{\mu_{n}: n \in \omega\right\}$ de cubiertas abiertas se dirá que es decreciente si $\mu_{n+1}<\mu_{n}$ para todo $n \in \omega$.

En primer lugar, observemos que si cada sucesión decreciente de $\omega$-cubiertas abiertas es especial, entonces cada sucesión de $\omega$-cubiertas abiertas es especial; para ésto, sea $\left\{\mu_{n}: n \in \omega\right\}$ una sucesión de $\omega$-cubiertas abiertas y hágase $\mu_{n}^{\prime}=\left\{U_{0} \cap \ldots \cap U_{n}: U_{i} \in \mu_{i}\right.$ para $\left.i \leq n\right\}$ para todo $n \in \omega$. Se checa fácilmente que cada $\mu_{n}^{\prime}$ es una $\omega$-cubierta abierta, así que por hipótesis la sucesión $\left\{\mu_{n}^{\prime}: n \in \omega\right\}$ es especial; puesto que $\mu_{n}^{\prime}<\mu_{n}$ para todo $n$, también es fácil checar que la sucesión $\left\{\mu_{n}: n \in \omega\right\}$ es especial. En segundo lugar, observemos que una sucesión decreciente $\left\{\mu_{n}: n \in \omega\right\}$ de $\omega$-cubiertas abiertas de $X$ es especial si contiene una subsucesión $\left\{\mu_{k_{i}}: i \in \omega\right\}$, donde $k_{i+1}>k_{i}$ para todo $i$, la cual es especial; para ver ésto, dados $U_{k_{i}} \in \mu_{k_{i}}$ tales que $\lim \left\{U_{k_{i}}: i \in \omega\right\}=X$, se construirá para cada $i \in \omega$ una sucesión $U_{k_{i}+1}, \ldots, U_{k_{i+1}-1}$ de manera recursiva y comenzando con $U_{k_{i+1}-1}$. Por ser $\mu_{k_{i+1}}<\mu_{k_{i+1}-1}$, existe $U_{k_{i+1}-1} \in \mu_{k_{i+1}-1}$ para el cual $U_{k_{i+1}} \subset U_{k_{i+1}-1}$; análogamente, existe $U_{k_{i+1}-2} \in \mu_{k_{i+1}-2}$ que cumple $U_{k_{i+1}-1} \subset U_{k_{i+1}-2}$ y así sucesivamente. Es claro que para la sucesión resultante $\left\{U_{i}: i \in \omega\right\}$ se verifica $\lim \left\{U_{i}: i \in \omega\right\}=X$, es decir, $\left\{\mu_{n}: n \in \omega\right\}$ es especial.

Verificar el enunciado significa probar que si $\left\{\gamma_{n}: n \in \omega\right\}$ es una sucesión de $\omega$-cubiertas abiertas de $X$, entonces es especial; por la primera observación del párrafo anterior se puede suponer que la sucesión es decreciente. Elíjase un conjunto $\left\{x_{i}: i \in \omega\right\} \subset X$ tal que $x_{i} \neq x_{j}$ si $i \neq j$; sea $\mu_{n}=\left\{U \backslash\left\{x_{n}\right\}: U \in \gamma_{n}\right\}$ para todo $n \in \omega$. Dado un conjunto finito $F$ en $X$, existe $N \in \mathbb{N}$ tal que $x_{i} \notin F$ para $i \geq N$; puesto que $\gamma_{N}$ es una $\omega$-cubierta de $X$, existe $U \in \gamma_{N}$ que cumple $F \subset U$, de donde $F \subset U \backslash\left\{x_{N}\right\} \in \mu_{N}$, así que $\bigcup\left\{\mu_{n}: n \in \omega\right\}$ es una $\omega$-cubierta abierta de $X$. Por (ii), existen sucesiones $\left\{k_{n}: n \in \omega\right\} \subset \mathbb{N}$ y $\left\{U_{n}: n \in \omega\right\}$ tales que $U_{n} \in \mu_{k_{n}}$ para todo $n$ y $\lim \left\{U_{n}: n \in \omega\right\}=X$. La sucesión $\left\{k_{n}: n \in \omega\right\}$ no puede estar acotada por algún $m \in \omega$ porque en ese caso, $\left\{x_{0}, \ldots, x_{m}\right\} \not \subset U_{n}$ para todo $n$, lo cual es una contradicción; ésto significa que se puede reordenar $\left\{k_{n}: n \in \omega\right\}$ de tal manera que la sucesión resultante $\left\{k_{\phi(n)}: n \in \omega\right\}$ sea creciente. Dado $x \in X$, existe $M \in \mathbb{N}$ tal que $x \in U_{n} \in \mu_{k_{n}}$ para $n>M$; dado que $k_{n}=k_{\phi\left(\phi^{-1}(n)\right)}$ para todo $n \in \omega$, se tiene que $x \in U_{\phi(j)} \in \mu_{k_{\phi(j)}}$ para $j>\max \left\{\phi^{-1}(n): n \leq M\right\}$, así que $\left\{\mu_{n}: n \in \omega\right\}$ tiene una subsucesión especial. Como $\mu_{n}<\gamma_{n}$ para todo $n$, la sucesión $\left\{\gamma_{n}: n \in \omega\right\}$ también tiene una subsucesión especial; por la segunda observación del párrafo anterior, la sucesión $\left\{\gamma_{n}: n \in \omega\right\}$ es especial.

(iii) $\Rightarrow\left(\right.$ i). Sean $A \subset C_{p}(X)$ y $f \in \bar{A}$; según la observación a la proposición 2.1.4, se puede 
suponer que $f=u$, donde $u$ es la función constante con valor 0 . Para cada $n \in \mathbb{N}$, sea $\gamma_{n}=$ $\left\{g^{-1}((-1 / n, 1 / n)): g \in A\right\}$; es fácil ver que cada $\gamma_{n}$ es una $\omega$-cubierta abierta de $X$. Por (iii), para todo $n \in \mathbb{N}$ se puede elegir $U_{n} \in \gamma_{n}$ de tal manera que $\lim \left\{U_{n}: n \in \mathbb{N}\right\}=X$; para cada $n \in \mathbb{N}$ existe $f_{n} \in A$ tal que $U_{n}=f_{n}^{-1}((-1 / n, 1 / n))$. Por la proposición 2.1.2, para probar que la sucesión $\left\{f_{n}\right\}$ converge a $u$ es suficiente mostrar que $\left\{f_{n}(x)\right\}$ converge a $u(x)=0$ para todo $x \in X$; así, sean $x \in X$ y $\epsilon>0$. Tómese $m \in \mathbb{N}$ para el cual $1 / m<\epsilon$ y $x \in U_{n}$ para $n \geq m$; entonces $\left|f_{n}(x)\right|<1 / n \leq 1 / m<\epsilon$ para todo $n \geq m$. Ésto prueba que $\left\{f_{n}(x)\right\}$ converge a 0 para todo $x \in X$.

2.2.19. Proposición. Para todo $X$, el espacio $C_{p}(X)$ es Fréchet-Urysohn si y sólo si $\left(C_{p}(X)\right)^{\omega}$ es Fréchet-Urysohn.

Demostración. Necesidad. Para probar que $\left(C_{p}(X)\right)^{\omega}$ es Fréchet-Urysohn normalmente tomaríamos cualesquiera $f \in\left(C_{p}(X)\right)^{\omega}$ y $A \subset\left(C_{p}(X)\right)^{\omega}$ tales que $f \in \bar{A}$, y mostraríamos que existe una sucesión $\left\{f_{n}\right\} \subset A$ que converge a $f$. Por la proposición 2.1.11, el espacio $\left(C_{p}(X)\right)^{\omega}=\prod_{t \in \omega} C_{p}\left(X_{t}, \mathbb{R}\right)$ donde $X_{t}=X$ para todo $t$, es homeomorfo al espacio $C_{p}\left(\bigoplus_{t \in \omega} X_{t}, \mathbb{R}\right)=C_{p}\left(\bigoplus_{t \in \omega} X_{t}\right)$, así que la observación a la proposición 2.1.4 nos libera de la restricción de que $f$ sea cualquier punto, permitiéndonos elegir uno especial. Por la proposición 2.1.10, el espacio $\left(C_{p}(X)\right)^{\omega}=\prod_{t \in \omega} C_{p}\left(X, Y_{t}\right)$ donde $Y_{t}=\mathbb{R}$ para todo $t$, es homeomorfo a $C_{p}\left(X, \mathbb{R}^{\omega}\right)$; si $\mu_{0} \in \mathbb{R}^{\omega}$ es la función constante con valor 0 , el punto elegido especialmente $f \in C_{p}\left(X, \mathbb{R}^{\omega}\right)$, será la función constante con valor $\mu_{0}$. Como $\mathbb{R}^{\omega}$ tiene una base numerable, existe una base local $\mathcal{B}_{0}=\left\{O_{n}: n \in \omega\right\}$ de $\mathbb{R}^{\omega}$ en $\mu_{0}$ para la cual $O_{n+1} \subset O_{n}$ para todo $n$.

Supongamos que $f \in \bar{A}$ y consideremos la familia $\gamma_{n}=\left\{g^{-1}\left(O_{n}\right): g \in A\right\}$ para todo $n \in \omega$; entonces cada $\gamma_{n}$ es una $\omega$-cubierta de $X$. Por el teorema 2.2.18(iii) para todo $n$ se puede elegir $U_{n} \in \gamma_{n}$ tal que $\lim \left\{U_{n}: n \in \omega\right\}=X$; para cada $n \in \omega$ existe $f_{n} \in A$ que cumple $U_{n}=f_{n}^{-1}\left(O_{n}\right)$.

Se probará que la sucesión $\left\{f_{n}\right\}$ converge a $f$; dados $x_{1}, \ldots, x_{n} \in X$, defínase el conjunto $W\left(x_{1}, \ldots, x_{n}\right)=\left\{g \in C_{p}\left(X, \mathbb{R}^{\omega}\right): g\left(x_{i}\right) \in O_{n}\right.$ para $\left.i \leq n\right\}$. Si $f \in U \in \tau\left(C_{p}\left(X, \mathbb{R}^{\omega}\right)\right)$, por ser $\mathcal{B}_{0}$ una base local de $\mathbb{R}^{\omega}$ en $\mu_{0}$ y por la proposición 2.1 .2 , existe $V=O\left(x_{1}, \ldots, x_{l} ; O_{n_{1}}, \ldots, O_{n_{l}}\right)$ tal que $f \in V \subset U$. Sea $k=\max \left\{l, n_{1}, \ldots, n_{l}\right\}$ y si $k>l$ hágase $x_{j}=x_{1}$ para $j=l+1, \ldots, k$; entonces $O_{k} \subset O_{n_{j}}$ para $j \leq l$ y $f \in W\left(x_{1}, \ldots, x_{k}\right) \subset V$. Puesto que $\lim \left\{U_{n}: n \in \omega\right\}=X$, existe $m \in \omega$ que satisface las condiciones $m>k$ y $\left\{x_{1}, \ldots, x_{k}\right\} \subset U_{n}=f_{n}^{-1}\left(O_{n}\right)$ para todo $n \geq m$; por tanto, $f_{n} \in W\left(x_{1}, \ldots, x_{k}\right)$ para $n \geq m$, de donde $\left\{f_{n}\right\}$ converge a $f$, así que $C_{p}\left(X, \mathbb{R}^{\omega}\right)$ es Fréchet-Urysohn.

Suficiencia. Dado que $C_{p}(X)$ se encaja como subespacio de $\left(C_{p}(X)\right)^{\omega}$, el enunciado es consecuencia de la proposición 1.3.1.

Nótese el contraste entre el ejemplo 1.3.4 por un lado y el corolario 2.2.13 y la proposición 2.2.19 por otro. 


\subsection{El teorema de Gerlits-Pytkeev}

En esta sección igual que en la anterior, nos restringimos a funciones cardinales con valores infinitos; además, el teorema principal muestra una vez más que si aplicamos a un espacio la operación $\mathcal{C}$, las relaciones entre algunas propiedades de $X$ aumentan de manera notable. En particular, en los espacios $C_{p}(X)$ coinciden la secuencialidad y la propiedad $k$.

2.3.1. Definición. La extensión de un espacio $X$ es el cardinal $\operatorname{ext}(X)=\sup \{|D|: D \subset X$ es cerrado y discreto\}.

El siguiente teorema se puede extender a la clase de los espacios conocidos como Lindelöf $\Sigma$, pero aquí sólo se requiere para espacios compactos.

2.3.2. Teorema (Baturov). Si $X$ es un espacio compacto, entonces para cada $Y \subset C_{p}(X)$ se cumple $\operatorname{ext}(Y)=l(Y)$.

Demostración. Dados una función $u: Z_{1} \rightarrow Z_{2}$ y $n \in \mathbb{N}$, se define $u^{n}$ como el producto cartesiano de las funciones $\left\{u_{i}: i=1, \ldots, n\right\}$ donde $u_{i}=u$ para $i \leq n$. Si $P$ es un conjunto, entonces $\operatorname{Fin}(P)=\{F \subset P: F \neq \emptyset$ y $|F|<\omega\}$. Para la familia $\mathcal{O}=\{(a, b): a<b, a, b \in \mathbb{Q}\}$ y cada $n \in \mathbb{N}$ sea $\mathcal{O}^{n}=\left\{O_{1} \times \cdots \times O_{n}: O_{i} \in \mathcal{O}\right.$ para $\left.i \leq n\right\}$; elíjase una numeración $\left\{O_{k}: k \in \omega\right\}$ de la familia $\bigcup\left\{\mathcal{O}^{n}: n \in \mathbb{N}\right\}$. Así, para cada $k \in \omega$ existen $m_{k} \in \mathbb{N}$ y $O_{1}^{k}, \ldots, O_{m_{k}}^{k} \in \mathcal{O}$ tales que $O_{k}=O_{1}^{k} \times \cdots \times O_{m_{k}}^{k}$. Para cada $x=\left(x_{1}, \ldots, x_{m_{k}}\right) \in X^{m_{k}}$ sea $\left[x, O_{k}\right]=\left\{f \in C_{p}(X): f^{m_{k}}(x) \in O_{k}\right\}$; si $\mathcal{B}_{k}=\left\{\left[x, O_{k}\right]: x \in X^{m_{k}}\right\}$, entonces $\mathcal{B}=\bigcup\left\{\mathcal{B}_{k}: k \in \omega\right\}$ es una base de $C_{p}(X)$ por la proposición 2.1.2.

Tomemos cualquier subespacio $Y \subset C_{p}(X)$. Se demuestra fácilmente que $\operatorname{ext}(Z) \leq l(Z)$ para todo espacio $Z$, así que para probar que $\operatorname{ext}(Y)=l(Y)$ basta mostrar que $l(Y) \leq \operatorname{ext}(Y)$, lo cual equivale a probar que $l(Y)>\kappa$ implica $\operatorname{ext}(Y)>\kappa$ para todo cardinal $\kappa$. Si $l(Y)>\kappa$, tómese $\mathcal{U} \subset \tau\left(C_{p}(X)\right)$ tal que $Y \subset \cup \mathcal{U}$ y ninguna subfamilia $\mathcal{V} \subset \mathcal{U}$ cumple $|\mathcal{V}| \leq \kappa$ y $Y \subset \cup \mathcal{V}$; claramente se puede suponer que $\mathcal{U} \subset \mathcal{B}$. Hágase $A_{k}=\left\{x \in X^{m_{k}}:\left[x, O_{k}\right] \in \mathcal{U}\right\}$ para todo $k \in \omega$; así la condición $Y \subset \cup \mathcal{U}$ es equivalente a:

(1) para cualquier $f \in Y$ existen $k \in \omega$ y $x \in A_{k}$ tales que $f^{m_{k}}(x) \in O_{k}$.

La afirmación de que ninguna subfamilia $\mathcal{V} \subset \mathcal{U}$ cumple $|\mathcal{V}| \leq \kappa$ y $Y \subset \cup \mathcal{V}$ equivale a:

(2) si $B_{k} \subset A_{k}$ y $\left|B_{k}\right| \leq \kappa$ para todo $k \in \omega$, entonces existe $f \in Y$ tal que $f^{m_{k}}\left(B_{k}\right) \cap O_{k}=\emptyset$ para cada $k$.

Elíjase $f_{0} \in Y$ y sea $B(k, 0)=\emptyset$ para todo $k \in \omega$. Supongamos que $0<\alpha<\kappa^{+}$y hemos hallado un conjunto $\left\{f_{\beta}: \beta<\alpha\right\} \subset Y$ y una familia $\{B(k, \beta): \beta<\alpha, k \in \omega\}$ con las siguientes propiedades:

(3) $B(k, \beta) \subset A_{k} \mathrm{y}|B(k, \beta)| \leq \kappa$ para todo $k \in \omega$ y $\beta<\alpha$;

(4) si $\gamma<\beta<\alpha$ entonces $B(k, \gamma) \subset B(k, \beta)$ para todo $k \in \omega$;

(5) para cualesquiera $\beta<\alpha, k \in \omega$ y $H \in F i n\left\{f_{\gamma}: \gamma<\beta\right\}$ el conjunto $u_{H}(B(k, \beta))$ es denso en $u_{H}\left(A_{k}\right)$ donde $u_{H}=\Delta\left\{f^{m_{k}}: f \in H\right\}: X^{m_{k}} \rightarrow \mathbb{R}^{m_{k} \cdot|H|}$ es un producto diagonal;

(6) $f_{\beta}^{m_{k}}(B(k, \beta)) \cap O_{k}=\emptyset$ para todos $\beta<\alpha$ y $k \in \omega$.

Para obtener $f_{\alpha}$, sea $F_{\alpha}=\left\{f_{\beta}: \beta<\alpha\right\}$ y fíjese $k \in \omega$; para cada $H \in F i n\left(F_{\alpha}\right)$ hágase 
$u_{H}=\Delta\left\{f^{m_{k}}: f \in H\right\}: X^{m_{k}} \rightarrow \mathbb{R}^{m_{k} \cdot|H|}$. De acuerdo con la desigualdad $w\left(u_{H}\left(A_{k}\right)\right) \leq \omega$, existe un conjunto numerable $B(H, k) \subset A_{k}$ tal que $u_{H}(B(H, k))$ es denso en $u_{H}\left(A_{k}\right)$; el conjunto $B(k, \alpha)=(\bigcup\{B(k, \beta): \beta<\alpha\}) \cup\left(\left\{B(H, k): H \in F i n\left(F_{\alpha}\right)\right\}\right)$ tiene cardinalidad a lo más $\kappa$. Por (2), existe $f_{\alpha} \in Y$ que cumple $f_{\alpha}^{m_{k}}(B(k, \alpha)) \cap O_{k}=\emptyset$ para todo $k \in \omega$; es fácil checar que las propiedades (3)-(6) se verifican para el conjunto $\left\{f_{\beta}: \beta \leq \alpha\right\}$ y la familia $\{B(k, \beta): \beta \leq \alpha$, $k \in \omega\}$, así que la inducción transfinita proporciona un conjunto $D=\left\{f_{\alpha}: \alpha<\kappa^{+}\right\}$y una familia $\left\{B(k, \beta): \beta<\kappa^{+}, k \in \omega\right\}$ que satisfacen las propiedades (3)-(6) para todo $\alpha<\kappa^{+}$.

Supongamos que $\beta<\alpha<\kappa^{+}$; se sigue de (4) y (5) que $f_{\beta}^{m_{k}}(B(k, \alpha))$ es denso en $f_{\beta}^{m_{k}}\left(A_{k}\right)$ para todo $k \in \omega$. Por $(1)$, se tiene que $f_{\beta}^{m_{k}}\left(A_{k}\right) \cap O_{k} \neq \emptyset$ y por eso $f_{\beta}^{m_{k}}(B(k, \alpha)) \cap O_{k} \neq \emptyset$ para algún $k \in \omega$. Por otro lado, $f_{\alpha}^{m_{k}}(B(k, \alpha)) \cap O_{k}=\emptyset$ según (6); en consecuencia, $f_{\alpha} \neq f_{\beta}$, así que $|D|=\kappa^{+}$.

Se probará que $D$ es cerrado y discreto por contradicción; para ello supongamos que $g$ es un punto de acumulación en $Y$ para $D$. Puesto que $X^{\omega}$ es compacto, se deduce que $l\left(X^{\omega}\right) \leq \omega$; luego, $t(Y) \leq t\left(C_{p}(X)\right) \leq \omega$ por el teorema 2.2.12. Por lo tanto, $g$ es un punto de acumulación de algún subconjunto numerable de $D$ y por ello, $\alpha=\min \left\{\beta<\kappa^{+}: g\right.$ es un punto de acumulación de $\left.F_{\beta}\right\}$ está bien definido; es claro que $\alpha$ es un ordinal límite. Existen $k \in \omega$ y $y \in A_{k}$ tales que $g \in\left[y, O_{k}\right]$; es evidente que $g$ también es un punto de acumulación de $G=F_{\alpha} \cap\left[y, O_{k}\right]$. Sean $K=\bigcap\left\{\left(f^{m_{k}}\right)^{-1}\left(f^{m_{k}}(y)\right): f \in G\right\}$ y $W=\left(g^{m_{k}}\right)^{-1}\left(O_{k}\right)$; entonces $K \neq \emptyset$ pues $y \in K$.

Si $K \backslash W \neq \emptyset$, tómese $x \in K \backslash W$ y nótese que $g^{m_{k}}(x) \notin O_{k}$, mientras $g^{m_{k}}(y) \in O_{k}$, de donde $g^{m_{k}}(x) \neq g^{m_{k}}(y)$; dado que $x \in K$, se obtiene $f^{m_{k}}(x)=f^{m_{k}}(y)$ para todo $f \in G$ lo cual contradice que $g \in \bar{G}$.

Si $K \subset W$, entonces el conjunto $K_{f}=\left(f^{m_{k}}\right)^{-1}\left(f^{m_{k}}(y)\right)$ es compacto para todo $f \in G$ y $y \in$ $\bigcap\left\{K_{f}: f \in G\right\}=K \subset W$; por [Engelking, corolario 3.1.5], existe un conjunto finito $H \subset G$ tal que $Q=\bigcap\left\{K_{f}: f \in H\right\} \subset W$. Nótese que si $u_{H}=\Delta\left\{f^{m_{k}}: f \in H\right\}$, entonces $Q=u_{H}^{-1}\left(u_{H}(y)\right)$; si ahora $u_{H}: X^{m_{k}} \rightarrow Y$ donde $Y=u_{H}\left(X^{m_{k}}\right)$, entonces $u_{H}$ es un mapeo perfecto.

Por [Engelking, teorema 1.4.13], existe $U \in \tau(Y)$ tal que $u_{H}(y) \in U$ y $u_{H}^{-1}(U) \subset W$. Sea $\gamma=\max \left\{\beta: f_{\beta} \in H\right\}$; entonces $\gamma<\mu=\gamma+1<\alpha$ pues $\alpha$ es un ordinal límite. Tenemos que $H \in F i n\left(F_{\mu}\right)$, de modo que $u_{H}(B(k, \mu))$ es denso en $u_{H}\left(A_{k}\right)$ por $(5)$; además, $u_{H}(y) \in U \cap u_{H}\left(A_{k}\right)$, por lo cual $\emptyset \neq U \cap u_{H}\left(A_{k}\right) \in \tau\left(u_{H}\left(A_{k}\right)\right)$. Según lo anterior, $u_{H}(B(k, \mu)) \cap U \neq \emptyset$ y por ello existe $z \in B(k, \mu)$ tal que $u_{H}^{-1}\left(u_{H}(z)\right) \subset W$; en particular, $z \in W$. Ésto implica que $g^{m_{k}}(z) \in O_{k}$.

De acuerdo con (6) se tiene que $f_{\mu}^{m_{k}}(B(k, \mu)) \cap O_{k}=\emptyset$; por (4) y (6), para cada ordinal $\beta$ tal que $\mu \leq \beta<\alpha$ se cumple $f_{\beta}^{m_{k}}(B(k, \mu)) \cap O_{k} \subset f_{\beta}^{m_{k}}(B(k, \beta)) \cap O_{k}=\emptyset$. En consecuencia, $f_{\beta}^{m_{k}}(z) \notin O_{k}$ siempre que $\mu \leq \beta<\alpha$, lo que muestra que $g \notin \overline{G \backslash F_{\mu}}$ y por ello $g$ es un punto de acumulación de $F_{\mu}$; puesto que $\mu<\alpha$, ésto contradice la elección de $\alpha$. Por tanto, $D$ es cerrado y discreto en $Y$, de donde $\operatorname{ext}(Y) \geq|D|=\kappa^{+}>\kappa$, tal como se quería probar.

2.3.3. Teorema (Pytkeev). Si $X$ es un espacio y ext $\left(X^{n}\right) \leq \omega$ para todo $n \in \mathbb{N}$, entonces se tiene que $t(K) \leq \omega$ para cada compacto $K \subset C_{p}(X)$.

Demostración. Sea $K$ un subespacio compacto de $C_{p}(X)$ y $E^{K}: X \rightarrow C_{p}(K)$ el mapeo de evaluación correspondiente; si $X^{\prime}=E^{K}(X)$, según el teorema 2.1.20(v), el espacio $K$ se encaja en $C_{p}\left(X^{\prime}\right)$ y por ello podemos suponer que $K \subset C_{p}\left(X^{\prime}\right)$. Es fácil probar que para cualquier función continua sobreyectiva $f: Y \rightarrow Z$ se cumple $\operatorname{ext}(Z) \leq \operatorname{ext}(Y)$; en particular, si $f$ es el producto cartesiano 
de las funciones $g_{i}$, en donde $g_{i}: X \rightarrow X^{\prime}$ coincide con $E^{K}$ para todo $i \in\{1, \ldots, n\}$. Por tanto, $\operatorname{ext}\left(\left(X^{\prime}\right)^{n}\right)=\operatorname{ext}\left(\left(g_{1} \times \cdots \times g_{n}\right)\left(X^{n}\right)\right) \leq \operatorname{ext}\left(X^{n}\right) \leq \omega$ para todo $n \in \mathbb{N}$, por hipótesis; puesto que $\left(X^{\prime}\right)^{n} \subset\left(C_{p}(K)\right)^{n}$, se sigue de la proposición 2.1.11 que se puede considerar a $\left(X^{\prime}\right)^{n}$ subespacio de $C_{p}\left(\bigoplus_{i \leq n} K_{i}\right)$ donde $K_{i}=K$ para $i \leq n$. Como $\bigoplus_{i \leq n} K_{i}$ es un espacio compacto, por el teorema 2.3.2 se tiene que $l\left(\left(X^{\prime}\right)^{n}\right) \leq \omega$ para todo $n$; en consecuencia, $t\left(C_{p}\left(X^{\prime}\right)\right) \leq \omega$ de acuerdo con el teorema 2.2.12. Por lo tanto, $t(K) \leq \omega$.

2.3.4. Definición. Un conjunto $A \subset C_{p}(X)$ se llama uniformemente denso en $C_{p}(X)$ si para cualesquiera $f \in C_{p}(X)$ y $\epsilon>0$, existe $g \in A$ tal que $|g(x)-f(x)|<\epsilon$ para todo $x \in X$.

2.3.5. Teorema (Tkachuk). Si $A \subset C_{p}(X)$ es uniformemente denso en $C_{p}(X)$, entonces se cumple la igualdad $t(A)=t\left(C_{p}(X)\right)$.

Demostración. Sea $\kappa=t(A)$; por $\kappa \leq t\left(C_{p}(X)\right)$, sólo debe probarse que $t\left(C_{p}(X)\right) \leq \kappa$. Por la proposición 2.2.11 y el teorema 2.2.12 es suficiente mostrar que para cualquier $\omega$-cubierta abierta $\mathcal{U}$ de $X$, existe una $\omega$-cubierta $\mathcal{U}^{\prime} \subset \mathcal{U}$ tal que $\left|\mathcal{U}^{\prime}\right| \leq \kappa$. De la definición 2.3.4, se prueba fácilmente que el traslado de un conjunto uniformemente denso en $C_{p}(X)$ también lo es; por ello, podemos suponer que $u \in A$, donde $u$ es la función constante de valor 1 . Dada $f \in A$, sea $S(f)=\{x \in X$ : $f(x) \geq 1 / 3\}$; para el conjunto $P=\{f \in A: S(f) \subset U$ para algún $U \in \mathcal{U}\}$ se probará que $u \in \bar{P}$. Sean $K=\left\{x_{1}, \ldots, x_{n}\right\} \subset X$ y $\epsilon>0$; entonces existe $U \in \mathcal{U}$ para el cual $K \subset U$. Tómese $h \in C_{p}(X)$ que cumpla $h(K)=\{1\}$ y $h(X \backslash U) \subset\{0\}$; como $A$ es uniformemente denso en $C_{p}(X)$, existe $f \in A$ tal que $|f(x)-h(x)|<\min \{1 / 3, \epsilon\}$ para todo $x \in X$. Por tanto, $f(x)<1 / 3$ para todo $x \in X \backslash U \mathrm{y}$ $|f(x)-1|<\epsilon$ para todo $x \in K$; ésto prueba que $S(f) \subset U$ y $f \in O\left(u ; x_{1}, \ldots, x_{n} ; \epsilon\right)$, es decir, $u \in \bar{P}$. Dado que $t(A) \leq \kappa$, existe $B \subset P$ que cumple $|B| \leq \kappa$ y $u \in \bar{B}$; para cada $f \in B$ elíjase $U_{f} \in \mathcal{U}$ para el cual $S(f) \subset U_{f}$. Dado un conjunto finito $F \subset X$, existe $f \in B$ tal que $|f(x)-1|<1 / 3$ para todo $x \in F$; en consecuencia $f(x)>2 / 3$ para todo $x \in F$, así que $F \subset S(f) \subset U_{f}$. El conjunto $\mathcal{U}^{\prime}=\left\{U_{f}: f \in B\right\} \subset \mathcal{U}$ resulta ser una $\omega$-cubierta de $X$ y $\left|\mathcal{U}^{\prime}\right| \leq \kappa$.

2.3.6. Definición. Se dice que un espacio $X$ tiene la propiedad $\varphi$, lo cual se denota por $X \vdash \varphi$, si para cada $\omega$-cubierta abierta $\mathcal{U}$ de $X$ que cumple $\mathcal{U}=\bigcup\left\{\mathcal{U}_{n}: n \in \mathbb{N}\right\}$ y $\mathcal{U}_{n} \subset \mathcal{U}_{n+1}$ para todo $n \in \mathbb{N}$, existe una familia $\left\{X_{n}: n \in \mathbb{N}\right\}$ de subconjuntos de $X$ tal que cada $X_{n}$ tiene por $\omega$-cubierta alguna $\mathcal{U}_{k_{n}} \mathrm{y} \lim \left\{X_{n}: n \in \mathbb{N}\right\}=X$.

2.3.7. Teorema (Gerlits-Nagy). Para todo espacio $X$, si $t\left(C_{p}(X)\right) \leq \omega$ y $X \vdash \varphi$, entonces $C_{p}(X)$ es Fréchet-Urysohn.

Demostración. Sea $\gamma$ una $\omega$-cubierta abierta de $X$; por la desigualdad $t\left(C_{p}(X)\right) \leq \omega$ aunada al teorema 2.2.12, se tiene que $l\left(X^{n}\right) \leq \omega$ para todo $n \in \mathbb{N}$, así que por la proposición 2.2.11, existe una $\omega$-cubierta $\mu=\left\{U_{n}: n \in \mathbb{N}\right\} \subset \gamma$. Sea $\mu_{n}=\left\{U_{j}: j \leq n\right\}$ para todo $n \in \mathbb{N}$; entonces $\mu=\bigcup\left\{\mu_{n}: n \in \mathbb{N}\right\}$ y $\mu_{n} \subset \mu_{n+1}$ para cada $n$. Puesto que $X \vdash \varphi$, existe una familia $\left\{X_{n}: n \in \mathbb{N}\right\}$ de subconjuntos de $X$ tal que cualquier $X_{n}$ tiene por una $\omega$-cubierta alguna $\mu_{k_{n}} \mathrm{y}$ $\lim \left\{X_{n}: n \in \mathbb{N}\right\}=X$. Se puede considerar, sin pérdida de generalidad que $k_{n}<k_{n+1}$ para cada

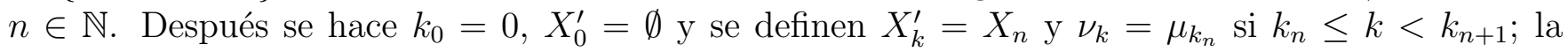
sucesión $\left\{X_{n}^{\prime}: n \in \omega\right\}$ cumple $\lim \left\{X_{n}^{\prime}: n \in \omega\right\}=X$ y cada $\nu_{n}$ es una $\omega$-cubierta de $X_{n}^{\prime}$. Ahora, una familia finita puede ser una $\omega$-cubierta de un conjunto si y sólo si el conjunto está contenido 
en algún elemento de la familia; por tanto, existe $V_{n} \in \nu_{n}$ para el cual $X_{n}^{\prime} \subset V_{n}$ para todo $n$. Es claro que $\left\{V_{n}: n \in \mathbb{N}\right\} \subset \gamma$ y $\lim \left\{V_{n}: n \in \mathbb{N}\right\}=X$; por el teorema 2.2.18, se concluye que $C_{p}(X)$ es Fréchet-Urysohn.

2.3.8. Lema. Si $X$ es un espacio $k$, entonces $t(X)=\sup \{t(K): K \subset X$ es compacto $\}$.

Demostración. Sean $\lambda=t(X)$ y $\kappa=\sup \{t(K): K \subset X$ es compacto $\}$; por la proposición 1.3.3, se cumple $\kappa \leq \lambda$. Para probar que $\lambda \leq \kappa$, sea $A \subset X$ tal que $A \neq \bar{A}$; por ser $X$ un espacio $k$, existe un conjunto compacto $K \subset X$ para el cual $A \cap K \neq \overline{A \cap K}$. La condición $t(K) \leq \kappa$ aunada a la proposición 1.2.7 implica que existe $B \subset A \cap K$ tal que $|B| \leq \kappa$ y $\bar{B} \backslash(A \cap K) \neq \emptyset$; puesto que $K$ es cerrado en $X$ se tiene que $\bar{B} \backslash A \neq \emptyset$ y como $B \subset A$, por la misma proposición concluimos que $\lambda \leq \kappa$.

2.3.9. Teorema (Tkachuк). Dado un espacio $X$, supongamos que $A$ es uniformemente denso en $C_{p}(X)$. Si $A$ es un espacio $k$, entonces $C_{p}(X)$ es Fréchet-Urysohn.

Demostración. Se afirma que $\operatorname{ext}\left(X^{n}\right) \leq \omega$, para todo $n \in \mathbb{N}$; para probarlo supongamos que para alguna $n \in \mathbb{N}$ existe un conjunto cerrado discreto no numerable $D \subset X^{n}$. Dados $f \in A$ y $m \in \mathbb{N}$, sea $U(f, m)=\{x \in X: f(x)<m\}$; defínase $P_{m}=\left\{f \in A:\left|(U(f, m))^{n} \cap D\right| \leq m\right\}$. Si $h \in A \backslash P_{m}$, entonces existen puntos distintos $z^{1}, \ldots, z^{m+1} \in(U(h, m))^{n} \cap D ;$ si $z^{i}=\left(z_{1}^{i}, \ldots, z_{n}^{i}\right)$, se cumple $h\left(z_{k}^{i}\right)<m$ para todo $i \leq m+1$ y $k \leq n$. De la proposición 2.1.2, se deduce que $O(h)=\left\{f \in A: f\left(z_{k}^{i}\right)<m\right.$ para cada $i \leq m+1$ y $\left.k \leq n\right\} \in \tau(h, A)$. Para cualquier $f \in O(h)$ se tiene que $\left\{z^{1}, \ldots, z^{m+1}\right\} \subset(U(f, m))^{n} \cap D$, así que $O(h) \cap P_{m}=\emptyset$ y por lo tanto $P_{m}$ es cerrado en A.

Considérese el conjunto $P=\bigcup\left\{P_{m}: m \in \mathbb{N}\right\}$ y sea $w \in A$ tal que $|w(x)| \leq 1 / 2$ para todo $x \in X$; entonces $U(w, m)=X$ para todo $m \in \mathbb{N}$, por lo cual cada conjunto $(U(w, m))^{n} \cap D$ no es numerable, de donde $w \notin P$. Sin embargo, $w \in \bar{P}$; para verlo toménse $K=\left\{x_{1}, \ldots, x_{l}\right\} \subset X$ y $\epsilon>0$. Por ser $D$ cerrado y discreto en $X^{n}$, existe $V \in \tau(K, X)$ y $k \in \mathbb{N}$ para los cuales $\left|V^{n} \cap D\right| \leq k$. Elíjase $f \in C_{p}(X)$ que cumpla $f|K=w| K$ y $f(X \backslash V)=\{k+1\}$; sean $\delta=\min \{1 / 4, \epsilon\}$ y $g \in A$ tales que $|g(x)-f(x)|<\delta$ para todo $x \in X$. Tenemos que $g(x)>k$ para cada $x \in X \backslash V$ y $|g(x)-w(x)|<\epsilon$ para todo $x \in K$, lo que implica que $g \in O\left(w ; x_{1}, \ldots, x_{l} ; \epsilon\right) \cap P_{k}$, y por ello $w \in \bar{P}$.

Por lo tanto, $P$ no es cerrado en $A$; luego existe un conjunto compacto $C \subset A$ tal que $C \cap P$ no es cerrado en $C$. Dado que cada $P_{m}$ es cerrado en $A$, se obtiene $C \not \subset P_{1} \cup \ldots \cup P_{l}$ para todo $l \in \mathbb{N}$; por eso existe una subsucesión $\left\{P_{m_{l}}\right\}$ de $\left\{P_{m}\right\}$ tal que $P_{m_{l}} \cap C \neq \emptyset$ y $m_{l+l}>m_{l}$ para todo $l$. Elíjase $f_{l} \in P_{m_{l}} \cap C$ para todo $l$; puesto que $|D|>\omega>\left|D \cap\left(U\left(f_{l}, m_{l}\right)\right)^{n}\right|$ para todo $l$, se tiene que $X^{n} \backslash \bigcup\left\{\left(U\left(f_{l}, m_{l}\right)\right)^{n}: l \in \mathbb{N}\right\} \neq \emptyset$. Tómese $z=\left(z_{1}, \ldots, z_{n}\right) \in X^{n} \backslash \bigcup\left\{\left(U\left(f_{l}, m_{l}\right)\right)^{n}: l \in \mathbb{N}\right\}$; entonces la sucesión $\left\{\sup \left\{f_{l}\left(z_{i}\right): 1 \leq i \leq n\right\}: l \in \mathbb{N}\right\}$ no está acotada. Según la definición 2.1.18 y la proposición 2.1.20, se tiene que $e_{z_{i}}(C)=\left\{f\left(z_{i}\right): f \in C\right\}$ es un conjunto acotado en $\mathbb{R}$ para todo $i \leq n$, lo cual contradice lo anterior; en consecuencia, $\operatorname{ext}\left(X^{n}\right) \leq \omega$ para todo $n \in \mathbb{N}$.

Por el teorema 2.3.3, se tiene que $t(K) \leq \omega$ para cada conjunto compacto $K \subset C_{p}(X)$. Puesto que $A$ es un espacio $k$, por el lema 2.3.8, se obtiene $t(A) \leq \omega$; por el teorema 2.3.5, se deduce que $t\left(C_{p}(X)\right) \leq \omega$.

Para terminar la demostración basta verificar que $X \vdash \varphi$. Si ésto no es cierto, podemos elegir una $\omega$-cubierta abierta $\mathcal{U}=\bigcup\left\{\mathcal{U}_{n}: n \in \mathbb{N}\right\}$ del espacio $X$ tal que $\mathcal{U}_{n} \subset \mathcal{U}_{n+1}$ para todo $n \in \mathbb{N}$ y $\mathcal{U}$ 
refuta la hipótesis $X \vdash \varphi$. Sea $B_{n}=\left\{f \in A: \mathcal{U}_{n}\right.$ es una $\omega$-cubierta de $\left.U(f, n)\right\}$ para cada $n \in \mathbb{N}$; se verá que cada conjunto $B_{n}$ es cerrado en $A$. Para ello tómese $h \in A \backslash B_{n}$; como $\mathcal{U}_{n}$ no es una $\omega$-cubierta de $U(h, n)$, existe un conjunto finito $K \subset U(h, n)$ tal que $K \not \subset V$ para todo $V \subset \mathcal{U}_{n}$. Por la proposición 2.1.2, se deduce que $G=\{f \in A: f(x)<n$ para todo $x \in K\} \in \tau(h, A)$; además, $G \subset A \backslash B_{n}$.

Sea $B=\bigcup\left\{B_{n}: n \in \mathbb{N}\right\}$; se afirma que la función $w$ definida en el segundo párrafo de esta demostración verifica $w \in \bar{B} \backslash B$. Para probar que $w \notin B_{m}$ para todo $m \in \mathbb{N}$, nótese que $U(w, m)=$ $X$ para todo $m$ y por ello es suficiente checar que cada $\mathcal{U}_{m}$ no es una $\omega$-cubierta de $X$; de no ser así, tomaríamos $X_{n}=X$ para todo $n \in \mathbb{N}$ y obtendríamos $\lim \left\{X_{n}: n \in \mathbb{N}\right\}=X$, donde $\mathcal{U}_{m}$ es una $\omega$-cubierta de $X_{n}$ para todo $n$, lo cual contradice que $\mathcal{U}$ refuta a $X \vdash \varphi$. Para ver que $w \in \bar{B}$, tómense $K=\left\{x_{1}, \ldots, x_{l}\right\} \subset X$ y $\epsilon>0$; por ser $\mathcal{U}$ una $\omega$-cubierta de $X$, existen $m \in \mathbb{N}$ y $U \in \mathcal{U}_{m}$ que cumplen $K \subset U$. Elíjase $f \in C_{p}(X)$ tal que $f|K=w| K$ y $f(X \backslash U)=\{m+1\}$. Sean $\delta=\min \{1 / 4, \epsilon\}$ y $g \in A$ tales que $|g(x)-f(x)|<\epsilon$ para todo $x \in X$; entonces, $g(x)>m$ para todo $x \in X \backslash U \mathrm{y}|g(x)-w(x)|<\epsilon$ para todo $x \in K$, por lo cual $g \in W=O\left(w ; x_{1}, \ldots, x_{l} ; \epsilon\right)$. Dado que $U(g, m) \subset U$, se infiere que $g \in B_{m}$, así que $g \in W \cap B_{m}$, de donde $w \in \bar{B}$.

Por ser $A$ un espacio $k$, existe un conjunto compacto $F \subset A$ tal que $F \cap B$ no es cerrado en $F$; para cada $x \in X$ existe $n(x) \in \mathbb{N}$ que cumple $f(x)<n(x)$ para todo $f \in F$. Luego, si hacemos $X_{n}=\{x \in X: n(x) \leq n\}$ para todo $n \in \mathbb{N}$, se obtiene $X_{n} \subset X_{n+1}$ para todo $n \mathrm{y}$ $X=\bigcup\left\{X_{n}: n \in \mathbb{N}\right\}$; por eso $\lim \left\{X_{n}: n \in \mathbb{N}\right\}=X$. Puesto que $\mathcal{U}$ refuta a $X \vdash \varphi$ existe $m \in \mathbb{N}$ para el cual ninguna $\mathcal{U}_{k}$ es una $\omega$-cubierta de $X_{m}$; por tanto, si $n>m$ y $f \in B_{n}$, la familia $\mathcal{U}_{n}$ es una $\omega$-cubierta de $U(f, n)$ más no de $X_{m}$, por lo cual $X_{m} \backslash U(f, n) \neq \emptyset$. Si $x \in X_{m} \backslash U(f, n)$, entonces $f(x) \geq n>m$, así que $f \notin F$ por definición de $X_{m}$. Ésto muestra que $F \cap B_{n}=\emptyset$ para $n>m$; en consecuencia $F \cap B=\bigcup\left\{F \cap B_{i}: i \leq m\right\}$ es un conjunto cerrado. De la contradicción resultante se deduce que $X \vdash \varphi$; puesto que $t\left(C_{p}(X)\right) \leq \omega$ y $X \vdash \varphi$, por el teorema 2.3.7 se concluye que $C_{p}(X)$ es Fréchet-Urysohn.

2.3.10. Teorema (Gerlits-Pytkeev). Para todo espacio $X$, las siguientes condiciones son equivalentes:

(i) $C_{p}(X)$ es Fréchet-Urysohn;

(ii) $C_{p}(X)$ es secuencial;

(iii) $C_{p}(X)$ es un espacio $k$.

Demostración. (i) $\Rightarrow$ (ii). Es consecuencia del teorema 1.2.9.

(ii) $\Rightarrow$ (iii). Se sigue del lema 1.3.14(i).

(iii) $\Rightarrow$ (i). Se desprende del teorema 2.3.9, haciendo $A=C_{p}(X)$. 


\section{Conclusiones y perspectivas}

En esta tesis se estudiaron las propiedades de convergencia en dos contextos: en espacios topológicos generales y en espacios de funciones $C_{p}(X)$. Se prepararon las bases para trabajar con espacios de funciones; en particular, se demostraron teoremas de caracterización de carácter numerable, la propiedad de Fréchet-Urysohn y secuencialidad en términos de imágenes de espacios métricos. En espacios $C_{p}(X)$, se vio que la estructura algebraica mejora notablemente las propiedades de convergencia y que todas las propiedades consideradas, pueden caracterizarse en términos de ciertas propiedades de $X$; los métodos desarrollados permitieron llegar a la frontera de lo que se conoce presentando entre otras cosas, los teoremas de Baturov y de Gerlits-Pytkeev.

Otra conclusión es que las propiedades de convergencia tienen útiles aplicaciones en el ámbito de la Topología General. Por ejemplo, si se conoce la estrechez $t(X)$ de un espacio $X$, para determinar los puntos de acumulación de un subespacio $A$ de $X$, puede uno restringirse a subconjuntos "pequeños" de $A$. Asimismo, el hecho de que $A \subset X$ sea cerrado o no, puede caracterizarse en términos de $t(X)$. Por otra parte, el carácter de un espacio $X$ permite hacer estimaciones del tamaño de ciertos subconjuntos de $X$ : el de $\bar{A}$ para todo $A \subset X$ o del mismo espacio $X$, si $X$ es compacto.

Como este trabajo contiene los resultados más importantes sobre las propiedades clásicas de convergencia, puede servir de base para un estudio de propiedades más especializadas: la radialidad, la pseudoradialidad, existencia de bases locales conservativas y la propiedad W de Gruenhage; también hay espacios $C_{p}(X)$ especiales que podrían considerarse, como los $C_{p}(X,[0,1])$. Con relación al teorema de Gerlits, se sabe que $C_{p}(X)$ es radial si y sólo si es Fréchet-Urysohn, y que si $C_{p}(X,[0,1])$ es un espacio $k$, no necesariamente es Fréchet-Urysohn, lo cual se verifica para $X=\omega_{1}$ con la topología discreta; ésto último resulta más intrigante dado que $C_{p}(X)$ se encaja como subespacio de $C_{p}(X,[0,1])$ y éste es subespacio de $C_{p}(X)$, en el cual se cumple el teorema de Gerlits.

Cuando ya se tiene una buena visión de los resultados de punta y se manejan adecuadamente los métodos modernos de estudio, el siguiente paso es intentar cruzar la frontera del conocimiento. La manera más común de hacerlo es atacar los problemas abiertos en el área. A continuación se presentan diez problemas que muestran unas líneas de investigación de las propiedades de convergencia en espacios de funciones:

1. Supongamos que $C_{p}(X,[0,1])$ es secuencial, ¿será Fréchet-Urysohn?

2. Supongamos que $X$ es cero-dimensional y $C_{p}(X,\{0,1\})$ es secuencial, ¿será Fréchet-Urysohn?

3. El espacio $C_{p}([0,1])$ no puede tener un subespacio denso Fréchet-Urysohn, pero ¿hay un subespacio denso secuencial en $C_{p}([0,1])$ ?

4. Supongamos que $X$ es compacto y $C_{p}(X)$ tiene un subespacio denso Fréchet-Urysohn, ¿será $X$ disperso?

5. Supongamos que $C_{p}(X,[0,1])$ tiene un subespacio denso Fréchet-Urysohn, ¿tendrá $C_{p}(X)$ un subespacio denso Fréchet-Urysohn? 
6. Supongamos que $C_{p}(X,[0,1])$ tiene un subespacio denso secuencial, ¿tendrá $C_{p}(X)$ un subespacio denso secuencial?

7. El espacio $C_{p}(X,[0,1])$ tiene un subespacio denso de estrechez numerable, ¿lo tendrá $C_{p}(X)$ ?

8. Supongamos que $C_{p}(X)$ tiene un subespacio denso Fréchet-Urysohn, ¿lo tendrá el espacio $C_{p}(X) \times C_{p}(X) ?$

9. Supongamos que $X$ es compacto y $C_{p}(X)$ tiene un subespacio denso secuencial, ¿será $X$ disperso?

10. Supongamos que $X$ es un espacio compacto tal que $C_{p}(X)$ tiene un subespacio denso secuencial, ¿tendrá un subespacio denso Fréchet-Urysohn? 


\section{Bibliografía}

Arhangel'SKII, A.V.

[1987] A survey of $C_{p}$-theory, Questions and Answers in General Topology, 5 (1987), 1-109.

[1988] Some problems and lines of investigation in general topology, Comment. Math. Univ. Carolinae, 29:4 (1988), 611-629.

[1990] Problems in $C_{p}$-Theory, en: Open Problems in Topology, North Holland, Amsterdam, 1990, 603-615.

[1992a] Topological Function Spaces, Kluwer Academic Publishers, Dordrecht, 1992.

[1992b] $C_{p}$-Theory, en: Recent Progress in General Topology, North Holland, Amsterdam, 1992, 1-56.

[1998] Some observations on $C_{p}$-theory and bibliography, Topol. Appl., 89 (1998), 203-221.

Arhangel'skit, A.V. y Ponomarev, V.I.

[1984] Fundamentals of General Topology: Problems and Exercises, D. Reidel Publishing Company, 1984.

Arhangel'skiI, A.V. y SzePtycki, P.J.

[1997] Tightness in compact subspaces of $C_{p}$-spaces, Houston J. Math., 23:1 (1997), 1-7.

Arhangel'skit, A.V. y TKachenko M.

[2008] Topological Groups and Related Structures, Atlantis Press/World Scientific, 2008.

Baturov, D.P.

[1987] On subspaces of function spaces (en ruso), Vestnik Moskovsk. Univ., Math., Mech., 42:4 (1987), 66-69.

Burke, D.K.

[1984] Covering Properties, Handbook of Set-Theoretic Topology, K. Kunen y J.E. Vaughan, editores, Elsevier Science Publishers B.V., 1984, 347-422.

ENGELKING, R.

[1989] General Topology, Heldermann Verlag Berlin, 1989.

FremLin, D.H.

[1994] Sequential convergence in $C_{p}(X)$, Comment. Math. Univ. Carolinae, 35:2 (1994), 371-382.

Gerlits, J.

[1983] Some properties of $C(X)$, II, Topol. Appl., 15:3 (1983), 255-262.

Gerlits, J. y NAGY, Zs. 
[1982] Some properties of $C(X)$, I, Topol. Appl., 14:2 (1982), 151-161.

Gerlits, J., Nagy, Zs. y Szentmiklóssy, Z.

[1988] Some convergence properties in function spaces, en: General Topology and Its Relation to Modern Analysis and Algebra, Heldermann Verlag Berlin, 1988, 211-222.

Gruenhage, G.

[1976] Infinite games and generalizations of first countable spaces, General Topology and Appl., 6:3 (1976), 339-352.

JuHÁsz, I.

[1971] Cardinal Functions in Topology, Mathematical Centre Tracts, 34, Amsterdam, (1971).

[1980] Cardinal Functions in Topology-Ten Years Later, Mathematical Centre Tracts, 123 , (1980).

JuhÁsz, I. y Szentmiklóssy, Z .

[1992] Convergent free sequences in compact spaces, Proc. Amer. Math. Soc., 116:4 (1992), 1153-1160.

KrivoruchKo, A.I.

[1972] On the cardinality of the set of continuous functions, Soviet. Math. Doklady, 13 (1972).

[1975] The cardinality and density of spaces of mappings, Soviet. Math. Doklady, 16 (1975), 281-285.

MaLYKhin, V.I.

[1998] On subspaces of the set of sequential spaces (en ruso), Matem. Zametki, 64:3 (1998), 407-413.

[1999] $C_{p}(I)$ is not subsequential, Comment. Math. Univ. Carolinae, 40:4 , (1999), 785-788. Malykhin, V.I. y Shakhmatov, D.B.

[1992] Cartesian products of Fréchet topological groups and function spaces, Acta Math. Hungarica, 60 (1992), 207-215.

McCoy, R.A.

[1980a] A k-space function space, Int. J. Math. Sci., 3 (1980), 701-711.

[1980b] Function spaces which are k-spaces, Topology Proc., 5 (1980), 139-146.

Michael, E.

[1973] On $k$-spaces, $k_{R^{-}}$-spaces and $k(X)$, Pacific J. Math., 47:2 (1973), 487-498. Noble, N.

[1973] The density character of function spaces, Proc. Amer. Math. Soc., 42:1 (1974), 228-233.

Nyikos, N.

[1981] Metrizability and the Fréchet-Urysohn property in topological groups, Proc. Amer. Math. Soc., 83:4 (1981), 793-801. 
[1989] Classes of compact sequential spaces, Set Theory Appl., Lecture Notes in Math., 1401, Springer, Berlin, 1989, 135-159.

OKunev, O.G.

[1996] A remark on the tightness of products, Comment. Math. Univ. Carolinae, $37: 2$ (1996), 397-399.

OKunev, O.G.

[1997] On the Lindelöf property and tightness of products, Topology Proc, 22 (1997), 363-371. Pytkeev, E.G.

[1982a] On the tightness of spaces of continuous functions (en ruso), Uspehi Mat. Nauk., 37:1 (1982), 157-158.

[1982a] Sequentiality of spaces of continuous functions (en ruso), Uspehi Mat. Nauk., 37:5 (1982), 197-198.

[1992] On Fréchet-Urysohn property of spaces of continuous functions (en ruso), Trudy Mat. Inst. RAN, 193 (1992), 156-161.

TKACHUK, V.V.

[1986a] The spaces $C_{p}(X)$ : decomposition into a countable union of bounded subspaces and completeness properties, Topol. Appl., 22:3 (1986), 241-254.

[1986b] Approximation of $\mathbf{R}^{\mathbf{X}}$ with countable subsets of $C_{p}(X)$ and calibers of the space $C_{p}(X)$, Comment. Math. Univ. Carolinae, 27:2 (1986), 267-276.

[2003] Properties of function spaces reflected by uniformly dense subspaces, Topol. Appl., 132 (2003), 183-193.

[2010] A $C_{p}$-Theory Problem Book, Topological and Function Spaces, Springer, Problem Books in Mathematics, 2010.

Vidossich, G.

[1969] A remark on the density character of function spaces, Proc. Amer. Math. Soc., 22 (1969), 618-619.

[1970] Characterizing separability of function spaces, Invent. Math., 10:3 (1970), 205-208. 


\section{Índice}

anillos topológicos topológicamente isomorfos, 31

carácter

de un conjunto en un espacio, 1

de un espacio, 1

celularidad de un espacio, 23

compactación de Čech-Stone, 6

condensación, 1

conjunto

de funciones que genera una topología, 28

de funciones que separa puntos, 28

de funciones que separa puntos de conjuntos cerrados, 28

$\kappa$-cerrado, 6

uniformente denso en $C_{p}(X), 43$

densidad de un espacio, 33

erizo de Urysohn, 3

espacio

compacto diádico, 8

con la propiedad $\varphi, 43$

Fréchet-Urysohn, 5

secuencial, 6

estrechez

de un espacio, 6

de un punto en un espacio, 6

extensión de un espacio, 41

familia localmente finita, 23

funcional lineal, 29

multiplicativa, 29

$i$-peso de un espacio, 33

mapeo

de evaluación, 28

de restricción, 25 dual, 26

hereditariamente cociente, 16

pseudoabierto, 16

$\mathbb{R}$-cociente, 26

número de Lindelöf de un espacio, 34

peso

de un espacio, 33

de red de un espacio, 30

pseudocarácter

de un espacio, 1

de un punto en un espacio, 1

recta de Sorgenfrey, 3

red de un espacio, 30

$\Sigma$-producto de una familia de espacios, 8

sucesión

convergente, 5

libre de longitud $\kappa, 18$

$\omega$-cubierta de un espacio, 34 